\title{
Determinantes y perfiles de la participación laboral en Colombia en el periodo 2002-2013*
}

\author{
Recibido: 8 de agosto de 2014 - Aceptado: 17 de abril de 2015
}

Doi: dx.doi.org/10.12804/rev.econ.rosario.18.01.2015.01

\author{
Néstor Iván González-Quintero ${ }^{\dagger}$ \\ Nancy Aireth Daza-Báez \\ Departamento Nacional de Planeación
}

\section{Resumen}

Para establecer los determinantes de la participación laboral en Colombia, sobre una muestra de individuos con representatividad nacional, así como el aporte de cada uno de estos determinantes a la dinámica de la tasa de participación en el periodo 2002-2013, se estiman modelos tipo probit y se establecen perfiles de participación, a partir de las probabilidades estimadas, condicionadas para cuatro grupos de mujeres y hombres en diferentes rangos de edad. Se concluye que alcanzar niveles de educación superior, contar con un ingreso por pensión y la presencia de menores en el hogar son factores importantes para explicar tal dinámica.

Palabras clave: Participación laboral, fuerza de trabajo, mercado laboral en Colombia, modelos probit.

Clasificación JEL: J21, C25

* Se agradecen los valiosos comentarios de Gabriel Piraquive, Manuel Ramírez, Jesús Otero y del equipo técnico de la Dirección de Estudios Económicos del Departamento Nacional de Planeación (DNP); así como las valiosas observaciones y sugerencias de los evaluadores anónimos de este trabajo. Por la colaboración en una versión previa de este proyecto, parte de los documentos de trabajo "Archivos de Economía" del DNP, agradecemos a Nidia Esperanza Garavito C. Las opiniones expresadas por los autores en este documento son de su responsabilidad propia, y en ningún momento comprometen a aquellas del DNP.

+ Departamento Nacional de Planeación. Correo electrónico: ngonzalez@dnp.gov.co; dirección: Calle 26 \# 13-19 piso 18; Teléfonos 3158998545/3815000 ext. 1821.

‡ Departamento Nacional de Planeación. Correo electrónico: ndaza@dnp.gov.co

Para citar este artículo: González-Quintero, N. I., \& Daza-Báez, N. A. (2015). Determinantes y perfiles de la participación laboral en Colombia en el periodo 2002-2013. Revista de Economía del Rosario, 18(1), 5-59. doi: dx.doi.org/10.12804/rev.econ.rosario.18.01.2015.01 


\title{
Determinants and Profiles of Labor Force Participation in Colombia During 2002-2013
}

\begin{abstract}
In order to find the main determinants of labor participation in Colombia over a sample of individuals representative of the country's labor force; and to establish the relative importance of these determinants for the explanation of the observed dynamics of the labor participation rate in the period 2002-2013, probit models are estimate and, based on conditional probabilities of participation, participation profiles are constructed for four different groups of men and women established by age. To have some tertiary education; the presence of children in the household; and to be a pensioner, are key factors for understanding such observed dynamics.
\end{abstract}

Keywords: Labor participation, workforce, labor market in Colombia, Probit models. JEL Classification: J21, C25

\section{Determinantes e perfiles da participação laboral na Colômbia no período 2002-2013}

\begin{abstract}
Resumo
Para estabelecer os determinantes da participação laboral na Colômbia sobre uma amostra de indivíduos com representatividade nacional. Assim como o aporte de cada um destes determinantes à dinâmica da taxa de participação no período 2002-2013, se estimam modelos de tipo probit e se estabelecem perfiles de participação a partir das probabilidades estimadas condicionadas para quatro grupos de mulheres e homens em diferentes rangos de idade. Conclui-se que alcançar níveis de educação superior, contar com ingresso por pensão e a presença de menores no lar, são fatores importantes para explicar tal dinâmica.

Palavras-chave: Participação laboral, força de trabalho, mercado laboral na Colômbia, modelos probit.

Classificação JEL: J21, C25
\end{abstract}




\section{Introducción}

Dependiendo de los factores que impulsen su cambio, la tendencia de la tasa de participación laboral es un indicador del cambio en la capacidad productiva de la economía y, en el corto y mediano plazo, refleja la situación del mercado de trabajo, al analizarla junto con los cambios en las tasas de ocupación o desempleo (Juhn \& Potter, 2006), razones más que suficientes para hacer relevante el estudio de los determinantes de la decisión de participación.

Durante las últimas cuatro décadas, los cambios en la tasa de participación han venido generando una reducción en la brecha de participación entre hombres y mujeres, en gran parte de los países de América Latina (Arriagada, 1997). En el caso colombiano, dichos cambios han estado asociados a importantes variaciones de factores demográficos, culturales y económicos, pero, entre estos, lo más significativo ha sido la percepción, por parte de las diferentes cohortes de mujeres, de un incremento en el costo de la fecundidad asociado con el aumento en los retornos a la educación y a cambios en la estructura de los hogares, relacionados con procesos de decisión conjunta de la asignación del tiempo (Sánchez \& Núñez, 2003). Este factor ha generado cambios significativos en el patrón de participación laboral en Colombia. En las siete principales ciudades ${ }^{1}$, la participación femenina aumentó considerablemente entre 1950 y 1985, al pasar del 19\% al 39\% (Ribero \& Meza, 1997). De igual manera, en las diez ciudades más grandes ${ }^{2}$, la participación de las mujeres, entre los 18 y 65 años, se incrementó y pasó del 47\% en 1984 al 65\% en 2006 (Amador, et al., 2013).

En este contexto, y debido al diseño de las encuestas de hogares en Colombia, el análisis de los determinantes de la participación en el mercado de trabajo solo se ha llevado a cabo, esencialmente, sobre muestras de individuos en las ciudades principales, siendo la muestra más grande y reciente la utilizada por Amador, et al. (2013) para diez grandes ciudades en el periodo 1984-2006, aunque Arango, et al. (2003) utilizaron una muestra representativa del ámbito nacional para hombres y mujeres en 2002 y 2003. Por otra parte, y a pesar de los detallados análisis de la evolución de la oferta laboral por grupos, hecha por Sánchez y Núñez (2003), el ciclo de vida no ha sido tomado en cuenta solo mediante la introducción de la edad como una variable determinante para la decisión de participación de un grupo de individuos de todas las edades.

1 Barranquilla, Bogotá, Bucaramanga, Cali, Manizales, Medellín y Pasto.

2 Barranquilla, Bogotá, Bucaramanga, Cali, Cúcuta, Manizales, Medellín, Pasto, Pereira y Villavicencio. 
Utilizando datos representativos del total nacional en el periodo 2002-2013, en el presente estudio se estiman modelos empíricos tipo probit de determinantes de la decisión de participación. A partir del comportamiento observado en las tasas de participación de diferentes grupos de edad, el análisis se lleva a cabo, para las mujeres, en el rango de 25-59 años de edad; para mujeres y hombres menores de 19; para mujeres y hombres con edades entre los 20 y 24 y para mujeres y hombres mayores de 59. Esta evaluación mira con más cuidado las posibles diferencias que implican estar en diferentes fases del ciclo de vida, y distingue el efecto de los niveles educativos alcanzados, así como de cada uno de los menores dependientes en el hogar. En igual sentido, para el último grupo etario estudiado, se introduce el papel del cubrimiento de la seguridad social en pensiones.

El periodo de análisis escogido coincide con el periodo posterior a la crisis de 1999, observándose un comportamiento en forma de $U$ de la tasa de participación, la cual muestra un incremento secular después de 2007. Con el propósito de establecer el verdadero aporte de aquellos determinantes a esta dinámica, se crearon perfiles de participación para subgrupos representativos de cada uno de los sectores analizados, para los cuales se calculan probabilidades de participación condicionadas a determinantes específicos para explicar las diferencias en la probabilidad de participación durante todo el periodo de estudio.

Entre los resultados más relevantes, se encuentra que el haber alcanzado niveles de educación superior tiene una relación directa e importante con los incrementos en la tasa de participación de las mujeres, en especial para aquellas casadas, después de 2008. Este efecto es también significativo para comprender la participación de los mayores de 59 años, para los cuales contar con un ingreso de pensión explica gran parte de las diferencias en la probabilidad de intervenir en el mercado de trabajo. Este hallazgo pone en evidencia el papel de la capacidad de cubrimiento del sistema de seguridad social en explicar la dinámica de los indicadores del mercado de trabajo.

En el caso de los jóvenes, las reducciones observadas en las tasas de participación entre 2002 y 2005 pueden, en parte, ser explicadas por las mejoras laborales, en especial, en el caso de las mujeres menores de 19 años; mientras que, a partir de 2008, los incrementos en la tasa de participación femenina están asociados con los mayores niveles educativos alcanzados, la menor presencia de niños y la presión económica que los hombres tienen ante la presencia de estos últimos en el hogar. 


\section{Estudios de participación laboral en Colombia}

El incremento paulatino que ha presentado durante las últimas décadas la participación laboral femenina y el efecto que este ha tenido en la participación laboral en Colombia ha generado que el análisis de los determinantes de participación laboral sea un tema extensamente trabajado por la literatura económica nacional, la cual se puede dividir en dos grupos, de acuerdo con la fuente de información empleada. En primer lugar, se destacan los trabajos de Ribero y Meza (1997), Tenjo y Ribero (1998), Santamaría y Rojas (2001) y Arango y Posada (2002), quienes hacen uso de la Encuesta Nacional de Hogares (ENH); y, en segundo lugar, se encuentran los trabajos de Arango, et al. (2003) y Amador, et al. (2013), quienes utilizan datos de la Encuesta Continua de Hogares (ЕCH).

En el grupo que emplea la ENH, los diferentes autores estimaron modelos de elección binaria (probit o logit) sobre la población total, desagregada entre hombres y mujeres, de las siete principales ciudades. Ribero y Meza (1997) analizan los determinantes de la participación laboral para hombres y mujeres entre 1976 y 1995, empleando un pseudo-panel con variables de control demográficas (edad, educación, estado civil, número de niños en el hogar, jefatura de este, número de personas que viven allí, asistencia escolar, participación laboral del cónyuge) y económicas (ingresos del cónyuge o ingresos del hogar), encontrando que los principales determinantes para hombres y mujeres son jefatura del hogar, edad, educación y participación laboral del cónyuge. El estado civil, por su parte, afecta negativamente la participación de las mujeres, mientras que aumenta la de los hombres.

Tenjo y Ribero (1998) incorporan la tasa de desempleo familiar y la existencia de servicio doméstico como posibles determinantes de la participación de hombres y mujeres, desagregando entre solteros y casados, hombres jefe de hogar y mujeres esposas de jefe de hogar. En su estudio, los autores encuentran que ser jefe de hogar y estar casado aumenta la participación de los hombres, mientras que la de las mujeres se ve incrementada por la tasa de desempleo familiar. En el grupo de los casados, la educación es un determinante tanto para hombres como para mujeres, mientras que el servicio doméstico lo es solo para mujeres. La participación de los solteros está determinada por la edad y la tasa de desempleo familiar, en el caso de los hombres, y por la edad y la presencia de menores de 6 años, en el caso de las mujeres.

Siguiendo la misma línea de estudio, Santamaría y Rojas (2001) consideran que la existencia de personas con discapacidad en el hogar, la migración 
y el efecto ciudad contribuyen a explicar la participación de hombres y mujeres. Los resultados señalan que la educación es un determinante para ambos sexos, entretanto el servicio doméstico lo es para mujeres y la migración, para los hombres. Adicionalmente, se destaca el aumento de la tasa de desempleo y la caída del ingreso familiar como factores que aceleraron la participación femenina durante el periodo 1983-2000.

Con el fin de aportar nuevos elementos al análisis de los determinantes de la intervención en el mercado de trabajo, Arango y Posada (2002) realizan un análisis trimestral considerando el efecto riqueza (Variable dummy, que tiene en cuenta la tenencia de vivienda, ubicación-estrato e ingresos mensuales superiores a \$2500000) y la desagregación entre hombres y mujeres, comprometidos y no comprometidos. Los hallazgos muestran que la edad y la existencia de miembros del hogar desempleados aumentan la participación laboral de hombres y mujeres no comprometidos, mientras que la educación la incrementa para las mujeres comprometidas y no comprometidas. Por su parte, la variable riqueza disminuye la participación, como se esperaba.

Empleando datos de la ECH, Arango, et al. (2003) estiman el modelo propuesto inicialmente por Arango y Posada (2002) para el periodo comprendido entre el primer trimestre de 2000 y el segundo de 2002, para trece ciudades, en este encontraron que los determinantes de la participación laboral para ambos sexos, entre la ENH y la ECH, en los periodos seleccionados, no han cambiado mucho.

Por otra parte, Amador, et al. (2013) realizaron un estudio con sub-periodos de la ENH y la ECH para las diez ciudades más grandes, con el objetivo de entender mejor el cambio sufrido en la participación laboral femenina en el periodo 1984-2006. Mediante una descomposición de la tasa de participación, para observar si su evolución se ha dado por cambios en la composición de la población o en la tasa de participación de diferentes grupos de individuos (nivel educativo, fertilidad y estado civil), encontraron que el incremento en la participación laboral femenina estuvo influenciado por el aumento en la tasa de participación de las mujeres con bajo nivel educativo que tienen pareja (casadas o en unión libre), mientras que los cambios en la composición poblacional tuvieron un efecto mínimo.

\section{Datos}

En Colombia, las encuestas de hogares han sido los instrumentos utilizados para medir el comportamiento del mercado laboral. Sus preguntas buscan recolectar información sobre condiciones de empleo (si trabaja, en qué trabaja, cuánto 
gana, si cuenta con seguridad social en salud y/o pensión o si está buscando empleo), características generales de la población (sexo, edad, estado civil y nivel educativo) y fuentes de ingreso.

Con el propósito de realizar un análisis más completo de los determinantes de la participación en Colombia, se emplearon los datos proporcionados por la ECH y la Gran Encuesta Integrada de Hogares (GEIH) para el periodo 20022013, dado que proporcionan información representativa en el ámbito nacional. Es importante destacar que, antes de la ECH, el Departamento Administrativo Nacional de Estadística (DANE) realizó la ENH entre 1976 y 2000; sin embargo, esta logra cubrir para todo su periodo solo siete ciudades ${ }^{3}$.

En este sentido, la posibilidad de contar con una muestra representativa a escala nacional es una primera motivación para realizar el análisis de la situación de la participación laboral en el periodo que cubren estas dos encuestas. Sin embargo, los cambios mencionados en la metodología de recolección de la información hacen que los datos del periodo 2002-2005 (ЕCH) no sean estrictamente comparables con los del periodo 2007-2013 (GEIH). Un primer intento de homogeneización se realizó mediante un ajuste de las dos muestras a las medidas de población nacional, de acuerdo con la información del Censo 2005, empleando los factores de ajuste calculados por la Misión para el Empalme de Series de Empleo, Pobreza y Desigualdad (MESEP) para unir las series de Tasa Global de Participación (TGP), Tasa de Ocupación (TO) y Tasa de Desempleo (TD). Adicionalmente, algunos otros controles fueron utilizados en el ejercicio de estimación para tener en cuenta la interferencia del cambio metodológico mencionado, aunque el tamaño de las muestras no presenta cambios significativos.

Para efectos de este documento, la población objetivo es la población en edad de trabajar (PET), que en el periodo 2002-2005 representó, en promedio, el $76 \%$ (30918021 individuos) de habitantes, y en el periodo 2007-2013 representó, en promedio, el 78,4\% (34677603 individuos). En el primer caso, la representatividad se asocia con una muestra de 444309 habitantes, mientras que en el segundo, la muestra es de 645825 , tal y como se observa en la tabla 1, mediante la cual se determina la muestra y su expansión a la población dividida por

3 Una visión histórica de la implementación de las encuestas de hogares se encuentra en el apéndice A.

4 No se tiene en cuenta la información de 2006, ya que durante el primer semestre de este se recogió información mediante la ech y durante el segundo semestre, mediante la geih, sin tener un periodo de recolección de información en común; por lo tanto no es confiable agregar la información para obtener resultados sobre los datos anuales. Véase también el apéndice $\mathrm{A}$. 
género. Para cada individuo de este subconjunto de la población, se cuenta con las principales características generales y la estructura sociodemográfica y económica de su hogar, lo que permite realizar un análisis más completo de los determinantes de la participación laboral.

Tabla 1. Tamaño de muestra e indicadores principales del mercado laboral en la ECH y la GEIH

\begin{tabular}{llrr}
\hline & & $2002-2005$ & \multicolumn{1}{c}{$2007-2013$} \\
\hline PET & Expandido & $30,918,021$ & $34,677,603$ \\
& Muestra & 444,309 & 645,825 \\
Mujeres & Expandido & $15,867,649$ & $17,739,837$ \\
& Muestra & 238,888 & 349,138 \\
Hombres & Expandido & $15,050,372$ & $16,937,765$ \\
PEA & Muestra & 205,421 & 296,687 \\
& Expandido & $19,075,299$ & $21,488,172$ \\
Mujeres & & 274,816 & 393,532 \\
& Expandido & $7,843,725$ & $9,013,360$ \\
Hombres & Muestra & 123,719 & 180,862 \\
TGP & Expandido & $11,231,574$ & $12,474,812$ \\
Mujeres & Muestra & 151,097 & 212,671 \\
Hombres & & $61.7 \%$ & $62.0 \%$ \\
\hline
\end{tabular}

Fuente: elaboración propia con base en DANE-ECH y DANE-GEIH

\section{Hechos estilizados}

Previo al periodo 2002-2013, la economía colombiana y el mercado de trabajo habían pasado por una de sus más agudas crisis en la última mitad de los noventa. Hasta 2001, el mercado laboral presentó altas tasas de desempleo y fuertes flujos de salida de la inactividad a la actividad (mayormente hacia el desempleo), en especial, para los grupos de individuos jóvenes y/o menos calificados. Esta situación se presentó en paralelo con reducciones importantes 
en el ingreso real de los trabajadores y altos niveles de informalidad laboral (Núñez \& González, 2011).

A partir de 2002, en el periodo de recuperación de la economía, la situación del mercado laboral parece cambiar significativamente. La tasa de desempleo presenta una tendencia decreciente y pasa de un valor del 15,6\% en 2002 al $9,7 \%$ en 2013, situación que es acompañada por incrementos continuos en la tasa de ocupación, y que, en el mismo periodo, pasó del 52,6 \% al 58 \% (figura 1, panel a). En este contexto, la tasa global de participación mostró una dinámica muy particular. Como era de esperarse para este periodo, entre 2002 y 2008 se presenta una reducción del indicador, que pasó del 62,2\% al 58,5\%; sin embargo, a partir de 2009, la tendencia de la tasa vuelve a ser creciente hasta alcanzar, en 2013, un valor del 64,2\%. Esta dinámica en forma de $U$, observada para la tasa de participación nacional, se repite cuando se descompone la participación por género (figura 1, panel b).

Al descomponer el comportamiento del indicador por grupos de edad, surgen otros resultados interesantes. La comparación que se puede observar en la figura 2, entre lo que sucede en los periodos 2002-2005 y 2007-2013, permite señalar que, tanto para hombres como para mujeres con edad menor a 19 años, se ha presentado una reducción en los niveles relativos de participación. En el caso de las mujeres, la tasa de participación pasó de un promedio de $18,3 \%$, en el primer periodo, a 14,3\%, en el segundo, mientras que en el caso de los hombres, estos valores fueron de 29,6\% y 24,1\%, respectivamente. Por su parte, el incremento de la tasa de participación parece estar asociado con la dinámica de dicho indicador para mujeres y hombres con edades entre 19 y 24 años, las mujeres con edades entre 25 y 59 años, y mujeres y hombres con 60 años o más. Entre estos, se destacan los incrementos observados en la participación femenina del segundo grupo $(64,2 \%$ a $67,6 \%)$ y del tercero $(19,0 \%$ a $21,0 \%)$.

Es posible tener una primera visión de la asociación que existe entre la dinámica de la participación y las características de la población. La tabla 2 resume la evolución de la tasa de participación, distinguiéndola por la situación civil y el nivel de educación alcanzado a lo largo del periodo 2002-2013. Para los menores de 19 años y los individuos con edades entre 19 y 24 años, se presentó un incremento importante en la tasa de participación de mujeres y hombres que alcanzaron niveles de educación terciaria incompleta y, en el caso particular del segundo grupo, se destaca también la tendencia creciente de la participación de los hombres con algún nivel de secundaria o secundaria completa. 

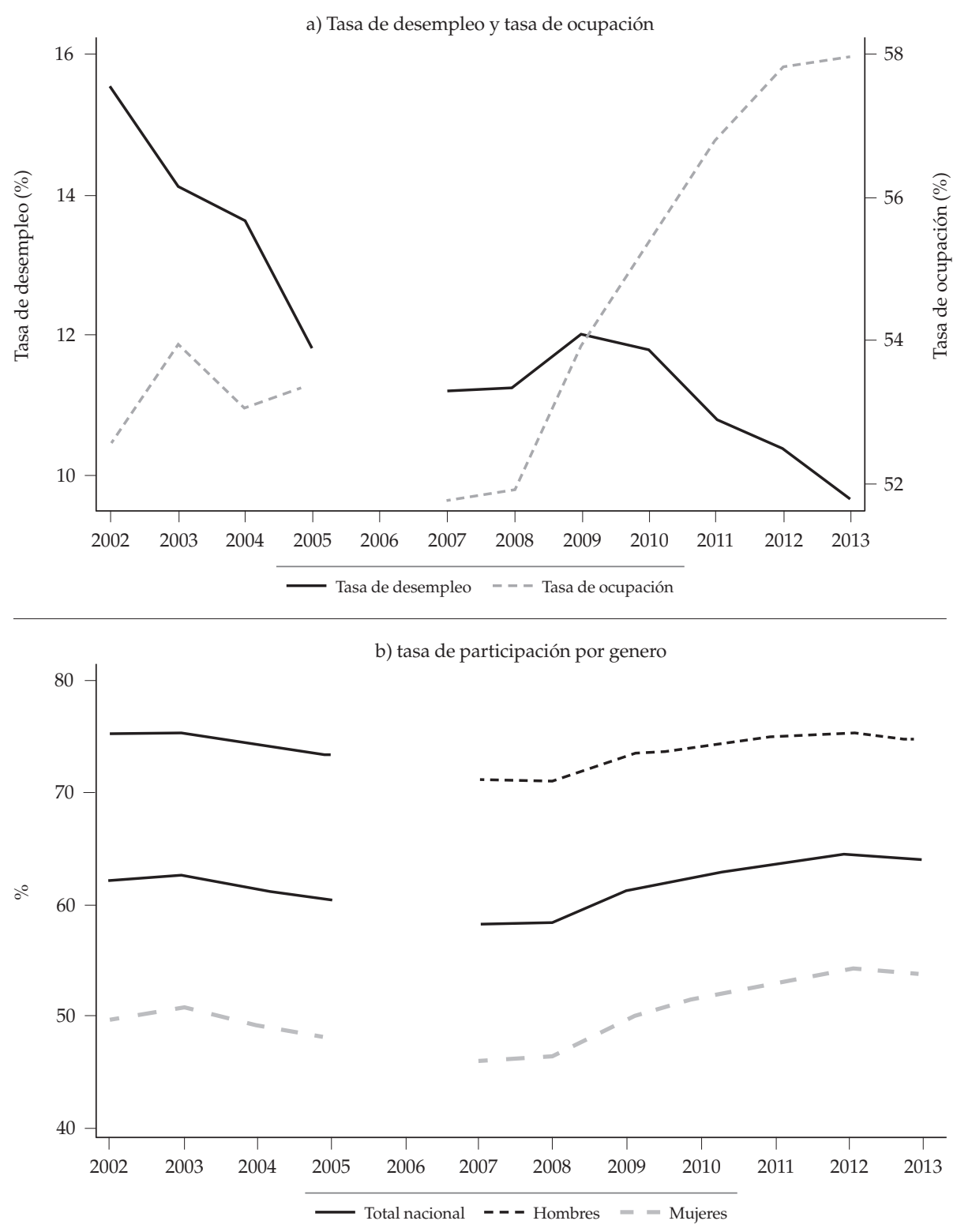

Figura 1. Tasas de desempleo, ocupación y participación en Colombia: 2002-2013 Fuente: elaboración propia con base en DANE-ECH y DANE-GEIH. 

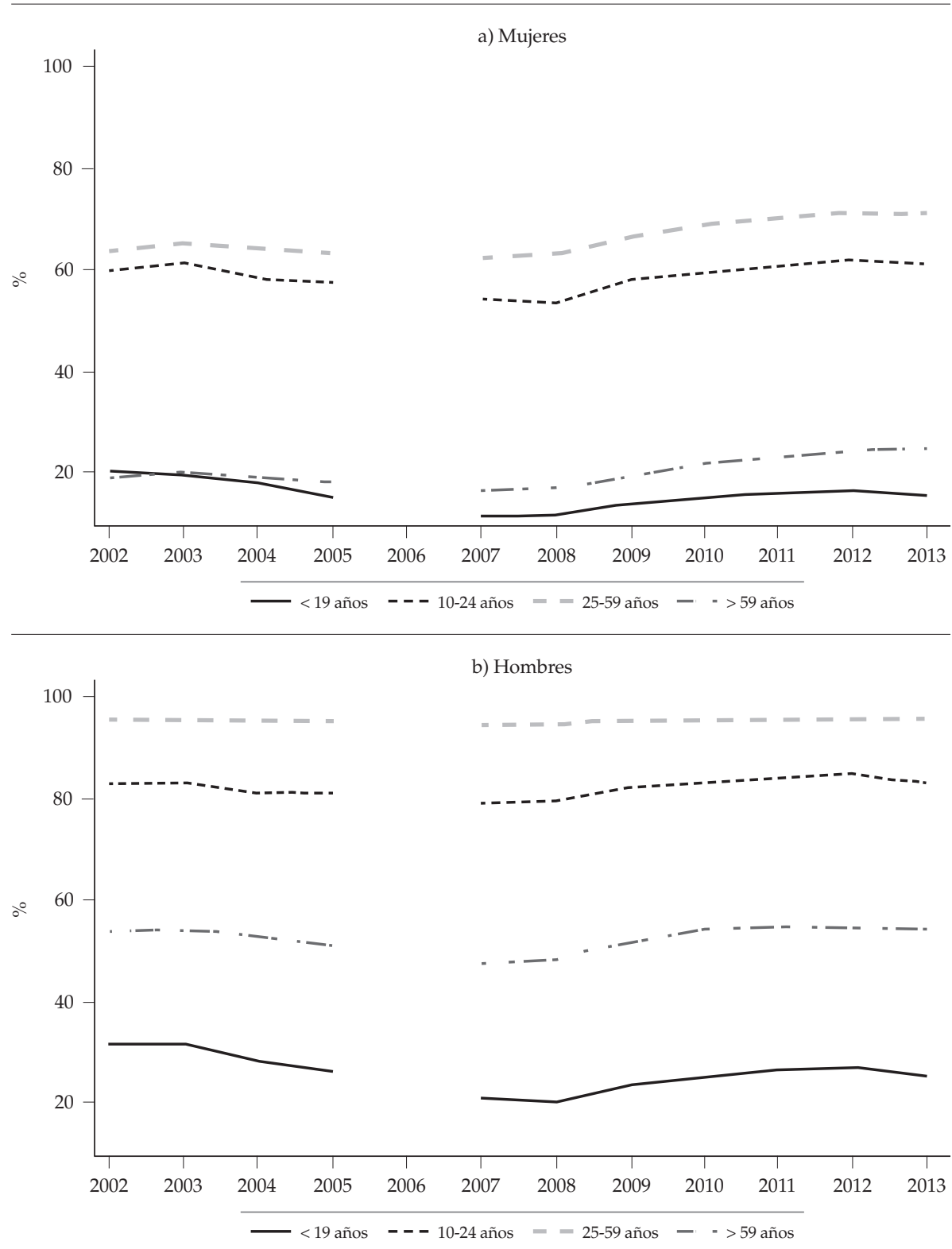

Figura 2. Tasa de participación laboral para hombres y mujeres por grupos de edad en el periodo 2002-2013

Fuente: Gran Encuesta Integrada de Hogares DANE-ECH (2002-2005) DANE-GEIH (2007-2013), cálculo de los autores. 


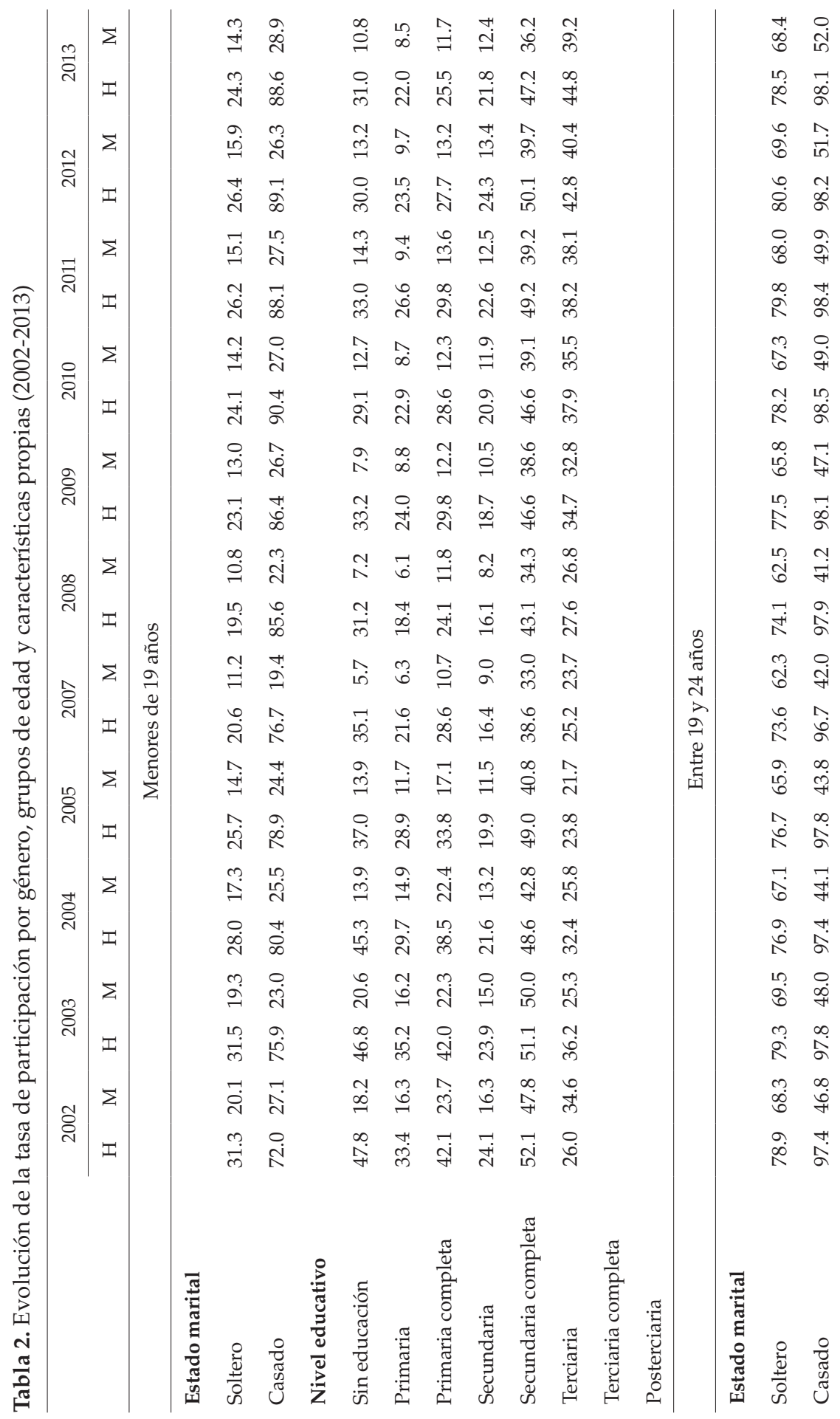




\begin{tabular}{|c|c|c|c|c|c|c|c|c|c|c|c|c|c|c|c|c|c|c|c|}
\hline \multirow{2}{*}{$\stackrel{\text { ते }}{\vec{N}}$} & $\Sigma$ & & $\begin{array}{l}\infty \\
\ddot{d}\end{array}$ & 品 & $\begin{array}{l}\text { Ln } \\
\text { fo }\end{array}$ & $\begin{array}{l}\sigma \\
\infty \\
\infty \\
\dot{\alpha}\end{array}$ & $\begin{array}{l}\circ \\
\text { రి }\end{array}$ & ○. & $\begin{array}{l}\widehat{\infty} \\
\infty\end{array}$ & ח̊ & & & $\underset{\infty}{\tilde{D}}$ & $\begin{array}{l}\circ \\
\stackrel{1}{0}\end{array}$ & & $\hat{f}$ & 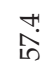 & $\begin{array}{l}\text { ర } \\
\text { రై }\end{array}$ & هُ \\
\hline & 工 & & $\begin{array}{l}\text { ำ } \\
\text { 年 }\end{array}$ & $\stackrel{\infty}{+}$ & 롱 & $\stackrel{\curvearrowright}{\stackrel{\infty}{\infty}}$ & $\stackrel{\infty}{\stackrel{\infty}{\infty}}$ & $\begin{array}{l}\infty \\
\infty \\
\infty\end{array}$ & $\begin{array}{l}0 \\
\stackrel{0}{\infty}\end{array}$ & $\begin{array}{l}\text { N } \\
\infty \\
\infty\end{array}$ & & & สู & ふু & & $\begin{array}{l}\infty \\
\stackrel{\infty}{\infty} \\
\infty\end{array}$ & $\begin{array}{l}\infty \\
\text { 여 }\end{array}$ & ֻु & $\begin{array}{l}\text { ?ु } \\
\text { हूँ }\end{array}$ \\
\hline \multirow{2}{*}{ స్తి } & $\Sigma$ & & $\begin{array}{l}\infty \\
\stackrel{\sim}{\sim}\end{array}$ & tr. & $\stackrel{\Upsilon}{f}$ & $\stackrel{9}{\overrightarrow{1}}$ & ڤై & $\begin{array}{l}m \\
\infty \\
\infty\end{array}$ & $\begin{array}{l}+4 \\
\infty \\
\infty\end{array}$ & \&̊. & & & $\stackrel{\infty}{\infty}$ & $\begin{array}{l}\circ \\
\stackrel{10}{0}\end{array}$ & & $\begin{array}{l}\stackrel{0}{+} \\
\stackrel{9}{9}\end{array}$ & $\begin{array}{l}0 \\
\infty \\
\infty\end{array}$ & + & ف \\
\hline & I & & $\overrightarrow{8}$ & 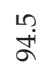 & 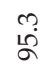 & $\begin{array}{c}N \\
\infty \\
\infty\end{array}$ & $\underset{\infty}{\sigma}$ & $\stackrel{n}{R}$ & $\begin{array}{l}\infty \\
1 \\
\infty\end{array}$ & $\ddot{\infty}$ & & & ๙ू & ঙু & & $\stackrel{\circ}{\circ}$ & బু & ֻُ & ชุ \\
\hline \multirow{2}{*}{$\overrightarrow{\bar{d}}$} & $\Sigma$ & & $\stackrel{m}{+}$ & $\stackrel{\circ}{\circ}$ & $\stackrel{\text { 号 }}{\stackrel{5}{f}}$ & $\stackrel{9}{\circ}$ & वें & 구요 & $\begin{array}{l}\circ \\
\stackrel{\infty}{\infty} \\
\infty\end{array}$ & ָ' & & & $\underset{\infty}{\stackrel{0}{ }}$ & $m_{3}^{3}$ & & $\hat{\gamma}$ & $\begin{array}{l}0 \\
\text { ○ें }\end{array}$ & ¿゙ & $\begin{array}{c}0 \\
\hat{n}\end{array}$ \\
\hline & I & & $\stackrel{\widehat{i}}{\Delta}$ & $\begin{array}{l}+! \\
\stackrel{2}{\alpha}\end{array}$ & $\begin{array}{l}\infty \\
\stackrel{\infty}{\alpha}\end{array}$ & $\begin{array}{l}0 \\
\infty \\
\infty \\
\infty\end{array}$ & $\begin{array}{c}\sim \\
\infty \\
\infty\end{array}$ & $\begin{array}{l}\infty \\
\dot{\delta} \\
0\end{array}$ & $\ddot{20}$ & $\begin{array}{l}\infty \\
\infty \\
\infty\end{array}$ & & & নू & م゚ & & $\stackrel{+}{\Delta}$ & ํํ & サேं & శู \\
\hline \multirow{2}{*}{ 올 } & $\Sigma$ & & $\stackrel{\llcorner}{\stackrel{i}{N}}$ & $\stackrel{\text { N }}{\not}$ & کั & $\begin{array}{l}0 \\
\dot{8} \\
10\end{array}$ & $\begin{array}{l}\infty \\
\stackrel{0}{0}\end{array}$ & $\stackrel{\overbrace{}}{\mathrm{d}}$ & $\underset{\infty}{\stackrel{N}{\infty}}$ & $\underset{\infty}{\stackrel{H}{\infty}}$ & & & $\underset{\infty}{\infty}$ & तु & & +̛ & $\begin{array}{l}\text { Nֶ } \\
\text { மீ }\end{array}$ & $\frac{0}{6}$ & 온 \\
\hline & I & & $\stackrel{\llcorner ?}{\stackrel{\llcorner}{6}}$ & 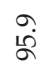 & $\begin{array}{l}0 \\
\text { o }\end{array}$ & $\stackrel{\circ}{\circ}$ & $\stackrel{\infty}{\stackrel{\infty}{\infty}}$ & サु & $\underset{+}{0}$ & $\begin{array}{l}0 \\
\dot{10} \\
\infty\end{array}$ & & & ๙ू. & ふু & & $\underset{\infty}{\wedge}$ & $\begin{array}{l}0 \\
\stackrel{0}{\circ}\end{array}$ & ชู & है \\
\hline \multirow{2}{*}{ ठे̀े } & $\Sigma$ & & ลે & $\stackrel{\curvearrowright}{\stackrel{f}{~}}$ & $\begin{array}{r}\mathscr{q} \\
\dot{q}\end{array}$ & $\begin{array}{l}\infty \\
\dot{q}\end{array}$ & ஜุ. & $\begin{array}{l}\infty \\
\dot{0} \\
i n\end{array}$ & $\begin{array}{l}\not{\infty} \\
\stackrel{\infty}{\infty}\end{array}$ & $\begin{array}{l}\infty \\
\stackrel{\infty}{\infty}\end{array}$ & & & $\underset{\infty}{N}$ & $\begin{array}{l}\text { மீ. } \\
\text { டें }\end{array}$ & & 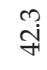 & ๙ิ่ & เి & वृ \\
\hline & I & & $\stackrel{\wedge}{\circ}$ & $\stackrel{\infty}{\alpha}$ & ڤౖ̊ & $\begin{array}{l}\widehat{N} \\
\stackrel{0}{\circ}\end{array}$ & $\stackrel{\stackrel{\llcorner}{\infty}}{\stackrel{\infty}{\infty}}$ & +艹 & $\begin{array}{l}10 \\
10 \\
\infty\end{array}$ & $\stackrel{\infty}{\wedge}$ & & & $\stackrel{\aleph}{\sigma}$ & ָㅗ & & $\underset{\infty}{\stackrel{\infty}{0}}$ & $\begin{array}{l}\text { ๙ू } \\
\text { బิ }\end{array}$ & ఫ̊ & 5 \\
\hline \multirow{2}{*}{$\stackrel{\infty}{\circledR}$} & $\Sigma$ & & $\begin{array}{l}\infty \\
\stackrel{\sim}{\sim}\end{array}$ & $\begin{array}{l}m \\
\ddot{m}\end{array}$ & 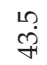 & $\underset{f}{+}$ & तु & $\begin{array}{l}\text { : } \\
10 \\
\text { மn }\end{array}$ & $\widehat{\hat{i}}$ & $\begin{array}{c}\text { di } \\
\text { d }\end{array}$ & & & $\stackrel{\substack{\infty \\
\infty}}{\infty}$ & ثิ & & $\begin{array}{l}\text { mo } \\
\text { లెं }\end{array}$ & $\begin{array}{l}0 \\
\stackrel{\infty}{+}\end{array}$ & تี & $\begin{array}{l}\infty \\
8 \\
1\end{array}$ \\
\hline & I & & તું & $\stackrel{10}{\alpha}$ & $\stackrel{g}{\dot{t}}$ & $\begin{array}{r}\widehat{\infty} \\
\infty\end{array}$ & $\begin{array}{l}\circ \\
110 \\
\infty\end{array}$ & $\begin{array}{l}m \\
10 \\
10\end{array}$ & $\underset{\infty}{\stackrel{10}{+}}$ & $\overrightarrow{\dot{\beta}}$ & & & ֻั & $\hat{\sigma}$ & & $\stackrel{\circ}{+}$ & ז̈ & ळू & 능 \\
\hline \multirow{2}{*}{. } & $\Sigma$ & & $\stackrel{0}{\stackrel{i}{\lambda}}$ & 官 & 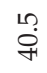 & $\begin{array}{l}+4 \\
\text { Pे }\end{array}$ & İ & 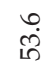 & $\begin{array}{l}10 \\
\infty \\
\infty\end{array}$ & $\stackrel{+}{2}$ & 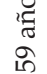 & & $\stackrel{N}{\aleph}$ & 울 & & $\stackrel{\infty}{+\infty}$ & $\hat{\sigma}$ & में & เి \\
\hline & I & & $\begin{array}{l}\infty \\
\dot{b}\end{array}$ & ふૂ & ๙ૂ & $\begin{array}{l}\stackrel{L}{\infty} \\
\infty \\
\infty\end{array}$ & $\underset{\infty}{\infty}$ & $\stackrel{\bar{i}}{\bar{n}}$ & $\overrightarrow{2}$ & $\vec{p}$ & $\overrightarrow{\stackrel{2}{N}}$ & & $\begin{array}{l}\stackrel{10}{\infty} \\
\infty\end{array}$ & ڤై & & $\begin{array}{l}+1 \\
\infty\end{array}$ & $\begin{array}{l}\text { } \\
\text { ti }\end{array}$ & İ & $\begin{array}{l}\infty \\
\dot{\sigma}\end{array}$ \\
\hline \multirow{2}{*}{ 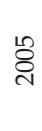 } & $\Sigma$ & & $\stackrel{\infty}{\stackrel{+}{+}}$ & $\stackrel{10}{\underset{F}{+}}$ & 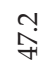 & ڤें & $\vec{\infty}$ & $\begin{array}{l}\text { } \\
\text { bे }\end{array}$ & $\begin{array}{l}\text { L? } \\
\text { م) }\end{array}$ & $\underset{\infty}{\stackrel{10}{+\infty}}$ & 氙 & & $\stackrel{\circ}{\circ}$ & $\begin{array}{l}0 \\
10 \\
10\end{array}$ & & $\overrightarrow{\dot{p}}$ & $\ddot{q}$ & 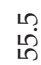 & 7 \\
\hline & $I$ & & बें & 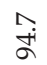 & 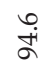 & $\begin{array}{l}\sigma \\
\dot{\infty}\end{array}$ & 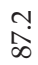 & 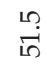 & $\begin{array}{l}\hat{\phi} \\
\varnothing\end{array}$ & ì & & & $\stackrel{\vec{\sigma}}{\sigma}$ & $\begin{array}{l}\infty \\
ٌ \\
\circ\end{array}$ & & $\begin{array}{l}\widehat{\wedge} \\
\infty \\
\infty\end{array}$ & $\begin{array}{l}\stackrel{10}{~} \\
\stackrel{\alpha}{\alpha}\end{array}$ & $\begin{array}{l}\infty \\
\text { வूं }\end{array}$ & 으 \\
\hline \multirow[b]{2}{*}{ હ্ণ } & $\Sigma$ & & $\begin{array}{l}\text { तु } \\
\text { ले }\end{array}$ & $\underset{f}{+}$ & $\begin{array}{l}\text { No } \\
\text { o }\end{array}$ & $\widehat{\widehat{ก}}$ & $\begin{array}{l}0 \\
\infty \\
0\end{array}$ & 迥 & $\underset{\infty}{\stackrel{M}{\infty}}$ & $\frac{\sigma}{\dot{\sigma}}$ & & & $\underset{1}{-\infty}$ & 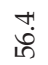 & & 궁 & 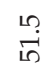 & $\begin{array}{l}\text { lo } \\
\text { டீ }\end{array}$ & 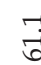 \\
\hline & $I$ & & $\stackrel{0}{\stackrel{R}{R}}$ & $\ddot{\alpha}$ & $\begin{array}{l}0 \\
\dot{\alpha}\end{array}$ & $\begin{array}{l}\infty \\
\infty \\
\infty\end{array}$ & ○ें & กొ & $\begin{array}{l}\infty \\
100 \\
\infty\end{array}$ & $\widehat{\widetilde{\sigma}}$ & & & $\vec{\sigma}$ & $\begin{array}{l}\circ \\
\text { Na }\end{array}$ & & $\begin{array}{l}0 \\
\stackrel{\infty}{0}\end{array}$ & $\begin{array}{l}\text { டे } \\
\text { Lू }\end{array}$ & $\begin{array}{l}m \\
\text { مू }\end{array}$ & $\begin{array}{l}\infty \\
12\end{array}$ \\
\hline \multirow{2}{*}{ రి } & $\Sigma$ & & ঙָ & $\stackrel{m}{g}$ & $\begin{array}{l}0 \\
10 \\
10\end{array}$ & 芦 & $\stackrel{\widetilde{N}}{\mathrm{~N}}$ & $\underset{\text { Ḣ }}{+1}$ & $\begin{array}{l}\circ \\
\dot{\infty}\end{array}$ & $\stackrel{+}{\stackrel{2}{N}}$ & & & 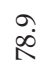 & $\begin{array}{l}n \\
\infty \\
\infty \\
\infty\end{array}$ & & $\begin{array}{l}\text { டे } \\
\text { If }\end{array}$ & $\stackrel{m}{\tilde{n}}$ & $\underset{\infty}{m}$ & $\stackrel{0}{8}$ \\
\hline & $I$ & & $\underset{\infty}{\vec{\infty}}$ & $\begin{array}{l}\text { ஜू } \\
\text { బू }\end{array}$ & $\begin{array}{l}\stackrel{\sigma}{~}{ }_{\alpha}\end{array}$ & $\begin{array}{l}0 \\
\infty \\
\infty\end{array}$ & $\begin{array}{l}\stackrel{1}{1} \\
\stackrel{\infty}{\infty}\end{array}$ & 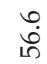 & $\stackrel{\widehat{\hat{\alpha}}}{\hat{\infty}}$ & 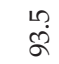 & & & $\stackrel{\curvearrowright}{\sigma}$ & নু & & ळे & $\begin{array}{l}10 \\
\text { ஜ̊ }\end{array}$ & ڤి & $\begin{array}{l}0 \\
\text { ठ̊ }\end{array}$ \\
\hline \multirow{2}{*}{ ఠి } & $\Sigma$ & & $\begin{array}{l}\circ \\
\stackrel{i}{d}\end{array}$ & $\underset{f}{+}$ & $\begin{array}{l}\ddot{N} \\
\text { مी }\end{array}$ & $\stackrel{n}{n}_{n}^{n}$ & $\stackrel{\wedge}{\wedge}$ & స్ & $\stackrel{\circ}{\circ}$ & ஷ̊ & & & $\stackrel{\infty}{\stackrel{\infty}{\Lambda}}$ & $\begin{array}{l}\hat{\varnothing} \\
\llcorner\end{array}$ & & $\stackrel{\overbrace{}}{f}$ & $\frac{0}{i n}$ & $\begin{array}{l}\infty \\
10 \\
\text { ம் }\end{array}$ & $\overrightarrow{3}$ \\
\hline & $I$ & & $\stackrel{\circ}{\stackrel{+}{\Lambda}}$ & ન્" & $\begin{array}{l}m \\
\mathscr{0}\end{array}$ & $\hat{\infty}$ & $\begin{array}{l}\stackrel{\circ}{\infty} \\
\infty\end{array}$ & $\begin{array}{l}\text { m. } \\
\text { in }\end{array}$ & $\vec{\infty}$ & $\begin{array}{l}\text { !n } \\
\stackrel{\infty}{\infty}\end{array}$ & & & $\begin{array}{l}\infty \\
\dot{\sigma}\end{array}$ & م̊ & & ஹ̊. & $\begin{array}{l}\grave{\alpha} \\
\grave{\alpha}\end{array}$ & ஜ̊ & ఠ̊ \\
\hline & & 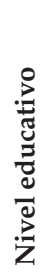 & 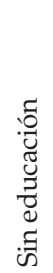 & 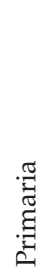 & 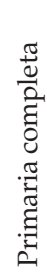 & 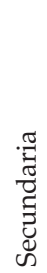 & 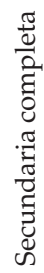 & & 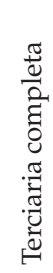 & 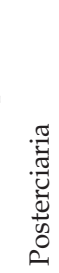 & & 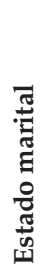 & 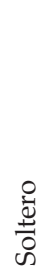 & $\begin{array}{l}\frac{8}{8} \\
\text { J } \\
\tilde{J} \\
\tilde{J}\end{array}$ & 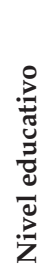 & 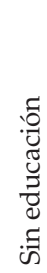 & 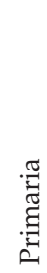 & 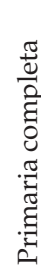 & 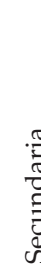 \\
\hline
\end{tabular}




\begin{tabular}{|c|c|c|c|c|c|c|c|c|c|c|c|c|c|c|c|c|c|c|c|}
\hline & $\Sigma$ & $\widehat{\aleph}$ & $\stackrel{\curvearrowright}{\infty}$ & $\stackrel{+1}{\circ}$ & $\hat{\sigma}$ & & & $\stackrel{+}{+}$ & $\begin{array}{l}0 \\
\text { ம் }\end{array}$ & & $\stackrel{\bar{N}}{\widehat{N}}$ & $\begin{array}{c}\text { N } \\
\stackrel{\text { N }}{N}\end{array}$ & $\stackrel{M}{\stackrel{M}{N}}$ & 롬 & $\begin{array}{l}\infty \\
\ddot{\sim}\end{array}$ & సี & $\begin{array}{l}0 \\
\infty \\
\infty\end{array}$ & 仓ิ & \\
\hline ત & $\Psi$ & $\begin{array}{l}\text { ț } \\
\text { ळ̆ }\end{array}$ & $\begin{array}{l}\text { டे } \\
\text { டू }\end{array}$ & 몽 & 苂 & & & 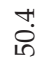 & $\begin{array}{l}9 \\
\stackrel{1}{\circ}\end{array}$ & & $\underset{+1}{+H}$ & $\begin{array}{l}\text { 官 } \\
\text { in }\end{array}$ & $\frac{\sigma}{i n}$ & ח? & $\begin{array}{l}a \\
\infty \\
\infty\end{array}$ & مُ & $\begin{array}{l}\hat{0} \\
\text { டे }\end{array}$ & $\begin{array}{l}\wedge \\
\infty \\
i n\end{array}$ & \\
\hline & $\Sigma$ & $\ddot{N}$ & $\underset{+}{\not{D}}$ & $\begin{array}{l}0 \\
\dot{\infty}\end{array}$ & $\begin{array}{l}\sigma \\
\dot{\Omega}\end{array}$ & & & 吕 & $\begin{array}{l}0 \\
\dot{v}\end{array}$ & & ڤ్రి & $\stackrel{\curvearrowright}{\text { ¿ }}$ & $\stackrel{\circ}{\underset{d}{d}}$ & $\begin{array}{l}\text { Lִ } \\
\stackrel{2}{N}\end{array}$ & 롱 & $\begin{array}{l}0 \\
\dot{\sim} \\
\dot{N}\end{array}$ & $\underset{\infty}{\infty}$ & $\stackrel{\circ}{\circ}$ & \\
\hline ๙ & $I$ & $\hat{\jmath}$ & $\begin{array}{l}+4 \\
\ddot{\alpha}\end{array}$ & 芴 & 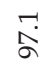 & & & $\underset{n}{+4}$ & $\vec{\circ}$ & & $\begin{array}{l}\text { N } \\
18\end{array}$ & 节 & $\begin{array}{l}0 \\
+ \\
\dot{10}\end{array}$ & $\begin{array}{l}10 \\
10 \\
10\end{array}$ & $\begin{array}{l}\infty \\
\dot{f}\end{array}$ & ํ. & ิิ & $\begin{array}{l}9 \\
\infty \\
\infty\end{array}$ & \\
\hline & $\Sigma$ & $\widetilde{N}$ & $\begin{array}{l}0 \\
\ddot{\infty}\end{array}$ & ळ. & $\ddot{\ddot{n}}$ & & & $\stackrel{\circ}{\mathrm{d}}$ & $\underset{d}{d}$ & & Nָ & $\stackrel{n}{\tilde{d}}$ & $\underset{\sim}{\sim}$ & $\stackrel{m}{\tilde{N}}$ & $\widehat{\curvearrowright}$ & $\begin{array}{l}\text { L } \\
\infty \\
\infty\end{array}$ & ๓ִ & サ! & \\
\hline ๙ૃ & $\Psi$ & $\stackrel{0}{\circ}$ & $\begin{array}{l}\infty \\
\text { ti }\end{array}$ & ڤे & $\stackrel{\infty}{\stackrel{\infty}{\alpha}}$ & & & 官 & ڤై & & تُ & ำ & ָె & 10. & 궁 & ơ & के & مิ & \\
\hline & $\Sigma$ & $\widehat{N}$ & $\begin{array}{l}\not \\
\infty \\
\infty\end{array}$ & 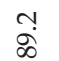 & ळे & & & $\overrightarrow{\ddot{N}}$ & 完 & & $\stackrel{n}{\sigma}$ & $\stackrel{+}{\mathbb{Z}}$ & $\hat{\dot{v}}$ & $\underset{+}{\sim}$ & ஓ̆ & $\stackrel{\sim}{\sim}$ & $\widehat{\ddot{\nu}}$ & $\begin{array}{l}\infty \\
\stackrel{\infty}{m}\end{array}$ & \\
\hline ๙ & $\Psi$ & サேं & $\begin{array}{l}+ \\
\text { H }\end{array}$ & ğ & 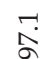 & & & $\stackrel{m}{n}$ & $\begin{array}{l}0 \\
\stackrel{1}{10}\end{array}$ & & ָ̦ & $\begin{array}{l}10 \\
\infty \\
\infty \\
10\end{array}$ & तิ & $\begin{array}{l}\circ \\
\stackrel{\dot{D}}{10}\end{array}$ & $\begin{array}{l}\infty \\
\infty \\
\infty\end{array}$ & $\begin{array}{l}\infty \\
\dot{\varphi} \\
\dot{f}\end{array}$ & $\vec{c}$ & $\begin{array}{l}\circ \\
\qquad 8 \\
0\end{array}$ & \\
\hline & $\Sigma$ & $\stackrel{n}{\sim}$ & 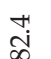 & $\ddot{\infty}$ & $\stackrel{\leftrightarrow}{\sigma}$ & & & $\begin{array}{l}\infty \\
\infty \\
\infty\end{array}$ & $\stackrel{\leftrightarrow}{\circ}$ & & 믈 & $\stackrel{\circ}{\circ}$ & $\underset{\infty}{\wedge}$ & $\begin{array}{l}10 \\
\infty \\
\sim\end{array}$ & $\stackrel{N}{N}$ & $\underset{\infty}{-1}$ & 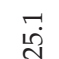 & ळे & \\
\hline ๙ & $I$ & $\begin{array}{l}\not ! \\
\text { ڤ̆ }\end{array}$ & $\begin{array}{l}\stackrel{+}{*} \\
\text { J }\end{array}$ & $\begin{array}{l}\not 1 \\
\text { ه̆ }\end{array}$ & $\begin{array}{l}\text { o } \\
\text { o }\end{array}$ & & & $\begin{array}{l}\stackrel{\circ}{q} \\
\stackrel{q}{q}\end{array}$ & $\overrightarrow{\dot{n}}$ & & $\begin{array}{l}m \\
\ddot{n} \\
0\end{array}$ & 苛 & ஜn & $\stackrel{L}{\mathscr{H}}$ & $\stackrel{\text { ?ִ }}{\not}$ & $\begin{array}{l}10 \\
\stackrel{9}{7}\end{array}$ & ت্ট் & - & \\
\hline & $\Sigma$ & $\begin{array}{l}0 \\
\stackrel{0}{\sigma}\end{array}$ & $\frac{1}{1}$ & $\begin{array}{l}\infty \\
\infty \\
\infty\end{array}$ & $\stackrel{H}{\mathrm{~J}}$ & & & $\vec{乛}$ & $\stackrel{?}{\stackrel{\rho}{\rho}}$ & & $\underset{\sim}{\stackrel{J}{ \pm}}$ & $\underset{\infty}{\infty}$ & ڤે & $\underset{\infty}{-\infty}$ & صृ & $\begin{array}{l}\stackrel{1}{\infty} \\
\stackrel{\infty}{\sigma}\end{array}$ & ิิ & $\underset{\mathfrak{f}}{\vec{f}}$ & \\
\hline 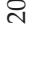 & $I$ & $\begin{array}{l}\mathscr{2} \\
\stackrel{2}{\alpha}\end{array}$ & ڤે & $\begin{array}{l}\text { Ḣ } \\
\text { ڤ̆ }\end{array}$ & ㅇ. & & & $\stackrel{\circ}{\stackrel{+}{+}}$ & 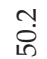 & & $\stackrel{\infty}{\stackrel{\infty}{f}}$ & $\frac{\sigma}{i n}$ & $\underset{+}{\stackrel{+}{+}}$ & ț & $\begin{array}{l}\text { ஸ̃ } \\
\ddot{\not}\end{array}$ & 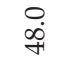 & 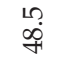 & $\begin{array}{l}\text { ํ. } \\
\text { กิ }\end{array}$ & \\
\hline & $\Sigma$ & $\overrightarrow{0}$ & ma & $\begin{array}{l}0 \\
\infty \\
\infty\end{array}$ & $\stackrel{\infty}{\sigma}$ & ๙ే & & $\stackrel{\vartheta}{\sigma}$ & $\begin{array}{l}\infty \\
\dot{\varphi}\end{array}$ & & $\begin{array}{l}\ddot{0} \\
\stackrel{10}{ }\end{array}$ & $\stackrel{\text { LP }}{\stackrel{\sim}{ح}}$ & $\begin{array}{l}\infty \\
\stackrel{\infty}{\stackrel{⿴}{2}}\end{array}$ & صृ & $\stackrel{m}{\stackrel{M}{2}}$ & $\underset{\text { ㄱ. }}{0}$ & $\begin{array}{l}\stackrel{0}{N} \\
\ddot{N}\end{array}$ & $\begin{array}{l}\text { aे } \\
\text { d. }\end{array}$ & \\
\hline 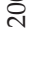 & $I$ & 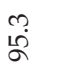 & $\begin{array}{l}\infty \\
\text { a }\end{array}$ & 음 & $\begin{array}{l}\text { H̆ } \\
\text { مे }\end{array}$ & $\begin{array}{l}\vec{D} \\
\mathscr{D}\end{array}$ & & $\vec{g}$ & $\stackrel{\leftrightarrow}{\dot{q}}$ & & 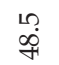 & $\begin{array}{l}\infty \\
\stackrel{0}{0} \\
\dot{n}\end{array}$ & $\underset{f}{\not{f}}$ & $\stackrel{\circ}{\underset{f}{f}}$ & $\vec{\infty}$ & Ln & $\stackrel{\llcorner}{\underset{f}{f}}$ & 官 & \\
\hline & $\Sigma$ & $\begin{array}{l}\infty \\
\infty \\
\infty\end{array}$ & $\underset{\infty}{\stackrel{0}{\infty}}$ & $\begin{array}{l}10 \\
\infty \\
\infty\end{array}$ & مू & $\stackrel{\vec{\pi}}{\Sigma}$ & & $\begin{array}{l}\stackrel{\infty}{0} \\
\infty\end{array}$ & $\underset{\sim}{\stackrel{\infty}{\infty}}$ & & 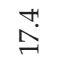 & ă & 邑 & $\stackrel{\circ}{\circ}$ & $\stackrel{\circ}{\stackrel{0}{\sim}}$ & $\widehat{\ddot{\nu}}$ & $\stackrel{\stackrel{一}{\sim}}{ }$ & $\begin{array}{l}\circ \\
\dot{f} \\
\dot{f}\end{array}$ & \\
\hline & $I$ & $\begin{array}{l}\text { ம் } \\
\grave{\alpha}\end{array}$ & ๙ે & ஜ̆ & ㄱ. & & & $\begin{array}{l}\text { no } \\
\text { in }\end{array}$ & $\begin{array}{l}\infty \\
\text { i } \\
\text { గn }\end{array}$ & & $\begin{array}{l}0 \\
\text { ti }\end{array}$ & $\begin{array}{c}\widehat{1} \\
10\end{array}$ & 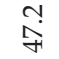 & $\vec{\sim}$ & 今. & $\begin{array}{l}\text { भִ } \\
\mathscr{f}\end{array}$ & $\begin{array}{l}\infty \\
\stackrel{0}{ } \\
\stackrel{1}{n}\end{array}$ & $\begin{array}{l}\hat{\infty} \\
\infty \\
i\end{array}$ & \\
\hline & $\Sigma$ & $\begin{array}{l}\text { gீ. } \\
\text { gे }\end{array}$ & হి & $\begin{array}{l}\infty \\
\infty \\
\infty\end{array}$ & $\frac{N}{\sigma}$ & & & $\stackrel{\circ}{\circ}$ & $\stackrel{\circ}{\circ}$ & & 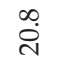 & $\stackrel{H}{\circ}$ & 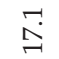 & $\begin{array}{l}0 \\
\infty \\
\sim\end{array}$ & $\stackrel{+!}{\triangle}$ & సิ & તิ & $\begin{array}{l}\infty \\
\dot{m} \\
\dot{m}\end{array}$ & \\
\hline & I & $\begin{array}{l}0 \\
\text { ஜें }\end{array}$ & $\begin{array}{l}o \\
\text { di }\end{array}$ & ğ & 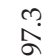 & & & $\begin{array}{l}0 \\
\dot{0}\end{array}$ & 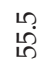 & & $\begin{array}{l}\infty \\
10 \\
10\end{array}$ & $\stackrel{\infty}{\stackrel{\infty}{0}}$ & न̈: & 今. & ঙ̣. & サr & 루요 & $\begin{array}{l}0 \\
\stackrel{0}{6}\end{array}$ & \\
\hline & $\Sigma$ & กี & $\underset{\infty}{+}$ & $\vec{\infty}$ & $\stackrel{\leftrightarrow}{\sigma}$ & & & กั & $\stackrel{0}{\circ}$ & & $\stackrel{+}{\vec{d}}$ & $\tilde{\tilde{N}}$ & @ొ & $\begin{array}{l}0 \\
\infty \\
\sim\end{array}$ & $\stackrel{+}{0}$ & $\stackrel{\infty}{\text { సิ }}$ & 苞 & يें & \\
\hline & $I$ & ஜ̊ & 오 & $\widehat{\hat{\sigma}}$ & $\stackrel{N}{N}$ & & & $\begin{array}{l}+4 \\
\infty \\
+\infty\end{array}$ & $\begin{array}{l}0 \\
\text { மீ }\end{array}$ & & $\begin{array}{l}\text { न゙் } \\
\text { மீ }\end{array}$ & ت̆ & 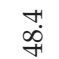 & $\begin{array}{l}\infty \\
\dot{f} \\
\dot{f}\end{array}$ & ㅇ.ᅮㅇ. & חृ & $\begin{array}{l}0 \\
\stackrel{+}{+1}\end{array}$ & $\begin{array}{l}n \\
\text { तె }\end{array}$ & \\
\hline & $\Sigma$ & $\stackrel{-}{-}$ & $\hat{\infty}$ & $\begin{array}{l}\stackrel{9}{\infty} \\
\infty\end{array}$ & $\hat{\sigma}$ & & & $\stackrel{\sigma}{\square}$ & $\stackrel{\sigma}{\sigma}$ & & $\begin{array}{l}\mathscr{L} \\
\infty \\
\infty\end{array}$ & $\begin{array}{l}0 \\
\infty \\
\sim\end{array}$ & $\underset{\mathscr{0}}{0}$ & $\underset{\infty}{\infty}$ & $\begin{array}{l}\infty \\
\stackrel{\sim}{\sim}\end{array}$ & 오. & $\begin{array}{l}\stackrel{\circ}{\mathrm{j}} \\
\stackrel{f}{f}\end{array}$ & $\begin{array}{l}m \\
\ddot{m}\end{array}$ & \\
\hline & $I$ & $\begin{array}{l}\text { ஜें } \\
\text { هे }\end{array}$ & ส̊ & $\begin{array}{l}\text { L? } \\
\text { مू }\end{array}$ & $\begin{array}{l}\text { ọ } \\
\text { ă }\end{array}$ & & & $\begin{array}{l}\infty \\
\stackrel{\sim}{*}\end{array}$ & $\begin{array}{l}\infty \\
10 \\
10\end{array}$ & & $\stackrel{\ulcorner}{+}$ & $\begin{array}{l}\text { ஜ } \\
\text { மீ }\end{array}$ & तें & $\begin{array}{l}\stackrel{\circ}{\circ} \\
\stackrel{f}{*}\end{array}$ & $\begin{array}{l}\text { ò } \\
\text { fi }\end{array}$ & $\begin{array}{l}\overrightarrow{0} \\
\stackrel{0}{\circ}\end{array}$ & กิ & กั & \\
\hline & & 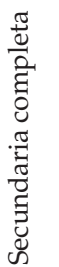 & 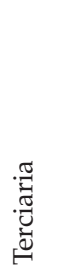 & 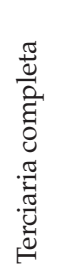 & 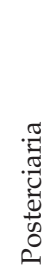 & & 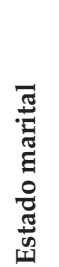 & 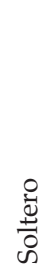 & $\begin{array}{l}\frac{0}{0} \\
\mathbb{E} \\
\tilde{U} \\
\tilde{U}\end{array}$ & 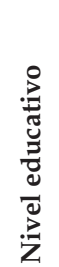 & 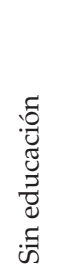 & 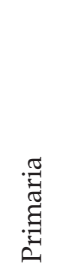 & 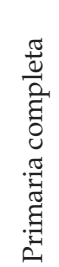 & 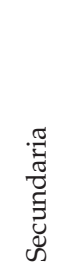 & 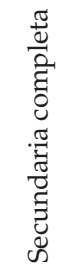 & 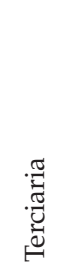 & 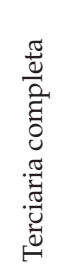 & 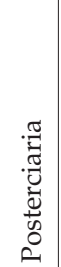 & \\
\hline
\end{tabular}


En las mujeres entre 19 y 59 años se observa también un incremento importante en la tasa de participación de las casadas y, entre estas, un aumento generalizado de las tasas de participación de las que tienen entre 25 y 59 años con educación igual o menor a la terciaria completa. Para el grupo de mayores de 59 años, las tendencias crecientes, especialmente a partir de 2010, se observan para hombres y mujeres con educación igual o superior a la terciaria incompleta, en donde se destaca la de aquellas con educación posterciaria.

\section{Determinantes de la participación laboral}

\subsection{Un modelo empírico de los determinantes de la participación laboral}

La naturaleza de los datos disponibles para entender la decisión de participación en la economía colombiana impone una restricción teórica importante al modelo empírico a utilizar en este análisis. Como no es posible seguir a los individuos a lo largo del tiempo, el modelo no podrá recoger muchos aspectos de la teoría del ciclo de vida y el efecto de la sustitución intertemporal en las decisiones de oferta de trabajo (Mulligan, 1998). Teniendo esto en consideración, se propone un modelo basado en la decisión estática de participación.

En la literatura empírica, la decisión de participación en el mercado de trabajo se considera la faceta extensiva del problema de oferta de trabajo del individuo (Heckman, 1993). Sea $h_{i t} \geq 0$ el tiempo de trabajo que podría utilizar un individuo $i$ en el mercado laboral en el periodo $t$, la extensión de la oferta de trabajo puede modelarse a través de la variable latente:

$$
y_{i t}^{*}=\left\{\begin{array}{l}
1 \text { si } h_{i t}>0 \\
0 \text { si } h_{i t}>0
\end{array}\right.
$$

Siguiendo los trabajos clásicos de Heckman (1974) y Heckman y Willis (1977), la disyuntiva entre ofrecer tiempo positivo de trabajo $y_{i t}^{*}=1$ y no ofrecerlo $y_{i t}^{*}=0$ depende de la comparación entre el beneficio y costo marginales de obtener un empleo. El salario de mercado $\omega_{i t}$ sería el beneficio, mientras que el costo estaría determinado por lo que se denomina el precio sombra del tiempo en la situación en que el individuo ofrece un tiempo nulo de trabajo $\mu_{i t}$. En este contexto, el comportamiento de la variable latente estaría determinado por una función $(f: \mathbb{R} \rightarrow\{0,1\})$ tal que: 


$$
y_{i t}^{*}=f\left(\omega_{i t}-\mu_{i t}\right)=\left\{\begin{array}{l}
1 \text { si } \omega_{i t}-\mu_{i t}>0 \\
0 \text { si } \omega_{i t}-\mu_{i t} \leq 0
\end{array}\right.
$$

El individuo ofrecerá tiempo positivo de trabajo solo si el beneficio marginal de participar exitosamente en el mercado laboral es superior al costo marginal; caso contrario, decidirá no participar. Desde el punto de vista empírico, tal y como lo señalan Heckman y Willis (1977), se presentan dos importantes problemas. En primer lugar, $\omega_{i t}$ es solo observable para aquellos individuos que participaron de manera exitosa en el mercado de trabajo, de manera que, si se utilizan los valores observados de esta variable, se estaría incurriendo en el denominado problema de sesgo de selección. En segundo lugar, $\mu_{i t}$ no es directamente observable para ningún individuo.

Una aproximación tipo Mincer (1974) resuelve parte del primer problema, y es posible decir que $\omega_{i t}$ es función de un conjunto de atributos $x_{1}$, entre los cuales se encuentran variables que representan el nivel educativo, la experiencia o la condición del mercado laboral, entre otras, probablemente, no observables. En el caso de $\mu_{i t}$ otro conjunto $z_{1}$ de atributos y situaciones personales pueden determinar dicho precio sombra, entre estos, la presencia de dependientes en el hogar, el ingreso de otros miembros del hogar y algunos otros no observables. A partir de la ecuación (1), y eliminando el subíndice de tiempo, se tiene que:

$$
y_{i}^{*}=f\left(x_{i}^{\prime} \beta+z_{i}^{\prime} \delta+\varepsilon_{i}\right)
$$

Asumiendo que el componente no observable tiene una distribución normal estándar en la ecuación (2), el modelo tipo probit de probabilidad de participación para el individuo $i$ se determina por:

$$
\begin{aligned}
\operatorname{Pr}\left(y_{i}^{*}=1\right) & =\operatorname{Pr}\left(x_{i}^{\prime} \beta+z_{i}^{\prime} \delta+\varepsilon_{i}>0\right) \\
& =\Psi\left(x_{i}^{\prime} \beta+z_{i}^{\prime} \delta\right) \\
& =(2 \pi)^{-1 / 2 e^{-\frac{\left(-x_{i}^{\prime} \beta+z_{i}^{\prime} \delta\right)^{2}}{2}}}
\end{aligned}
$$

\subsection{Variables y modelos estimados}

Como fue señalado en la sección 0 , los movimientos importantes en la participación laboral durante el periodo 2002-2013 se observaron para mujeres $(\mathrm{M}<19)$ y hombres $(\mathrm{H}<19)$ menores de 19 años; mujeres (M 19-24) y hombres (H 19-24) entre 19 y 24 años; mujeres entre 25 y 59 años, para las cuales se 
distinguió entre casadas (MC 25-59) y no casadas (MS 25-59); y mujeres $(\mathrm{M}>59)$ y hombres $(\mathrm{H}>59)$ mayores de 59 años. Se define a un individuo como casado si está legalmente ligado a una pareja bajo la institución del matrimonio o si cohabita con una pareja estable sin lazo legal; el individuo no casado sería aquel que no cumple con alguna de estas dos condiciones. La distinción es importante, en especial, para las mujeres, pues se espera que su uso del tiempo en actividades de mercado o no mercado sea muy dependiente de la decisión de formar un hogar.

Para cada individuo en la muestra utilizada, las variables que conforman los vectores $x_{i}$ y $z_{\mathrm{i}}$ se listan y definen en la tabla 3 . Conforman el primer vector edad, edad2 y el nivel de educación alcanzado, ya que determinan el beneficio marginal del tiempo en el mercado de trabajo a partir de su efecto sobre el salario potencial, evitando el problema de sesgo de selección. La variable edad2 representa el cuadrado de la edad y se incluye en los determinantes del salario para medir la no linealidad que se espera en la generación de ingreso laboral en el contexto del ciclo de vida económica del individuo. Por su parte, el precio sombra del tiempo utilizado en actividades de no mercado, medido por el vector $z_{i}$, es determinado, en primer lugar, por las decisiones de fertilidad y cuidado de dependientes recogidas en un conjunto de variables tipo dummy que indican la presencia y ausencia de menores o mayores no participantes en el hogar. En el primer caso, es de interés medir el efecto de la presencia de un menor adicional sobre la decisión de participación, razón por la cual se utilizan seis diferentes variables indicadoras para los grupos MC 25-59, MS 25-59, M> 59 y $\mathrm{H}>59$. Para los grupos de individuos menores de 25 años, debido a su corta edad, la decisión de fertilidad es representada por una única variable indicadora de la presencia de algún menor de seis años en el hogar. En el segundo caso, el número de mayores no participantes se incluye para todos los individuos con excepción de aquellos que conforman los grupos de mayores de 59 años.

Tabla 3. Variables independientes en los modelos de participación laboral

\begin{tabular}{|c|c|c|}
\hline Vector & Variable & Definición \\
\hline \multirow{5}{*}{$X_{i}$} & edad & Años de edad. \\
\hline & edad2 & Cuadrado de los años de edad. \\
\hline & $\sin e d u$ & $\begin{array}{l}\text { Variable con valor 0-1 que indica si el individuo no alcanzó algún } \\
\text { nivel educativo. }\end{array}$ \\
\hline & primaria & $\begin{array}{l}\text { Variable con valor 0-1 que indica si el individuo alcanzó algún nivel } \\
\text { educativo inferior a primaria completa. }\end{array}$ \\
\hline & primaria_comp & $\begin{array}{l}\text { Variable con valor 0-1 que indica si el individuo alcanzó únicamente la } \\
\text { primaria completa. }\end{array}$ \\
\hline
\end{tabular}




\begin{tabular}{|c|c|c|}
\hline Vector & Variable & Definición \\
\hline \multirow{4}{*}{$X_{i}$} & secundaria & $\begin{array}{l}\text { Variable con valor } 0-1 \text { que indica si el individuo alcanzó algún nivel de } \\
\text { secundaria inferior a secundaria completa. }\end{array}$ \\
\hline & terciaria & $\begin{array}{l}\text { Variable con valor } 0-1 \text { que indica si el individuo alcanzó algún nivel de } \\
\text { terciaria inferior a terciaria completa. }\end{array}$ \\
\hline & terciaria_comp & $\begin{array}{l}\text { Variable con valor } 0-1 \text { que indica si el individuo alcanzó únicamente la } \\
\text { terciaria completa. }\end{array}$ \\
\hline & post_terciaria & $\begin{array}{l}\text { Variable con valor } 0-1 \text { que indica si el individuo alcanzó algún nivel de } \\
\text { educación post-terciaria. }\end{array}$ \\
\hline \multirow{12}{*}{$Z_{i}$} & 1_menor_15 & $\begin{array}{l}\text { Variable con valor } 0-1 \text { que indica la presencia en el hogar de un sólo } \\
\text { menor con edad inferior a } 16 \text { años. }\end{array}$ \\
\hline & 2_menores_16 & $\begin{array}{l}\text { Variable con valor 0-1 que indica la presencia en el hogar de sólo dos } \\
\text { menores con edad inferior a } 16 \text { años. }\end{array}$ \\
\hline & 3_menores_16 & $\begin{array}{l}\text { Variable con valor 0-1 que indica la presencia en el hogar de sólo tres } \\
\text { menores con edad inferior a } 16 \text { años. }\end{array}$ \\
\hline & 4_menores_16 & $\begin{array}{l}\text { Variable con valor 0-1 que indica la presencia en el hogar de sólo cua- } \\
\text { tro menores con edad inferior a } 16 \text { años. }\end{array}$ \\
\hline & 5_menores_16 & $\begin{array}{l}\text { Variable con valor } 0-1 \text { que indica la presencia en el hogar de sólo cinco } \\
\text { menores con edad inferior a } 16 \text { años }\end{array}$ \\
\hline & 6_menores_16 & $\begin{array}{l}\text { Variable con valor } 0-1 \text { que indica la presencia en el hogar de seis o más } \\
\text { menores con edad inferior a } 16 \text { años. }\end{array}$ \\
\hline & menores_6_años & $\begin{array}{l}\text { Variable con valor } 0-1 \text { que indica la presencia en el hogar de al menos } \\
\text { un niño menor de } 6 \text { años de edad. }\end{array}$ \\
\hline & mayoresnop & $\begin{array}{l}\mathrm{N} \text { úmero de mayores de } 59 \text { años no participantes del mercado de tra- } \\
\text { bajo en el hogar. }\end{array}$ \\
\hline & desemp_h & Número de otros individuos desempleados en el hogar. \\
\hline & estudia & $\begin{array}{l}\text { Variable con valor 0-1 que indica si el individuo estudiaba en el mo- } \\
\text { mento de la encuesta. }\end{array}$ \\
\hline & log_ing_res_h & Logaritmo del ingreso total de los otros miembros del hogar. \\
\hline & pensionado & $\begin{array}{l}\text { Variable con valor 0-1 que indica si el individuo recibe algún ingreso } \\
\text { por concepto de pensión de jubilación. }\end{array}$ \\
\hline \multirow[b]{2}{*}{$(\mathrm{Ctrl})$} & casado & Variable con valor 0-1 que indica si el individuo está casado. \\
\hline & urbano & $\begin{array}{l}\text { Variable con valor } 0-1 \text { que indica si el individuo vive en cabecera o } \\
\text { centro poblado. }\end{array}$ \\
\hline
\end{tabular}

Fuente: Gran Encuesta Integrada de Hogares.

Se incluye, también, dentro del vector $z_{i}$, una variable que mide el número de otros desempleados en el hogar, la cual cuenta el total de personas desempleadas en el hogar del $i$-ésimo individuo, dejando a este por fuera, en caso de 
ser también desempleado. El efecto observable de esta variable sobre el precio sombra del tiempo utilizado en actividades de no mercado es, en principio, ambiguo. Siguiendo a Hotchkiss y Robertson (2006), es posible que entre mayor sea el número de desempleados en el hogar, menor sea el ingreso del mismo y, ante una situación de inactividad, el precio sombra se incremente incentivando al individuo a participar activamente en el mercado de trabajo (worker-added effect); pero también es posible que, si el número de desempleados en el hogar es importante, el individuo en situación de inactividad perciba esto como un signo de baja probabilidad de conseguir un empleo, lo cual reduce el precio sombra y desincentiva su participación en el mercado de trabajo (discouragedworker effect $)^{5}$.

Con el objetivo de controlar por zona geográfica, se incluye para todos los grupos una variable dicotómica que indica si el individuo vive o no en el área urbana. Además, dado que la decisión de vivir una vida de pareja no es exclusiva de las mujeres entre 25 y 59 años, el efecto de esta decisión sobre la de participación de los individuos del resto de grupos analizados, se mide a partir de una variable indicadora de estar casado. Finalmente, y con el propósito de controlar, por los cambios muestrales y metodológicos, las variables son complementadas con variables indicadoras para cada año, tomando como referencia 2013.

Los modelos de participación para cada uno de los grupos de individuos mencionados se basan en la ecuación (3), y la evaluación por máxima verosimilitud de cada uno de estos arroja como resultado el valor de los estimadores $\hat{\beta}$ y $\hat{\delta}$. Sin embargo, para establecer el efecto que cada una de las variables tiene sobre la probabilidad de participar, es necesario calcular los efectos marginales $\frac{\partial \Psi(\bullet)}{\partial x_{\ell i}}$ y $\frac{\partial \Psi(\Theta)}{\partial z_{n i}}$, donde $x_{\ell \mathrm{i}} \mathrm{y} z_{n i}$ denotan la variable $\ell$-ésima y $n$-ésima de los vectores $x_{i} \mathrm{y} z_{i}$, respectivamente.

5 Estos son los efectos que López (1985) denomina 'trabajador adicional' y 'trabajador desalentado', respectivamente. Dichos efectos se presentarían en épocas de crisis, y la razón detrás de ellos se encontraría en que, en el primer caso, la elasticidad-salario de la oferta extensiva de trabajo de los que él denomina trabajadores secundarios es, en valor absoluto, menor que la elasticidad-salario de la oferta extensiva de los trabajadores primarios; mientras que, en el segundo caso, se presentaría la situación opuesta. De acuerdo con el análisis de López, la elasticidad-salario de los trabajadores secundarios estaría altamente influenciada por el efecto de sustitución laboral intrafamiliar $(+)$ y los efectos tradicionales de ingreso (-) y sustitución (+). 


\subsection{Estimaciones}

Tabla 4 y tabla 5 resumen los modelos probit estimados para la muestra que representa el total nacional en el periodo 2002-2013. Entre los 20 y los 59 años, la probabilidad de participación se incrementa con la edad para cualquier individuo, esto asociado con el ciclo de vida y un mayor ingreso laboral esperado tabla 4); y el efecto marginal significativo y negativo de edad2 indica la presencia de la no linealidad esperada. Para los individuos con 60 años o más, el efecto de la edad es el contrario y la no linealidad no parece tener un efecto significativo, pues están en la fase descendente del ciclo de vida (modelos 7 y 8, tabla 5). En cuanto a los menores de 19 años, al estar en la fase inicial del ciclo de vida, la asociación entre la edad y la participación estimada es algo extraña. En el caso de las mujeres, el efecto marginal de edad es negativo y significativo, mientras que el de edad2 es positivo y significativo. Esto último, sin embargo, implica que en el margen la participación se acelera con la edad ${ }^{6}$.

Tabla 4. Determinantes de la participación laboral (Total Nacional).

Variable dependiente: participación

\begin{tabular}{lrrrr}
\hline & $(1)$ MS $25-59$ & $(2)$ MS $25-59$ & $(3) \mathrm{M} 19-24$ & $(4) \mathrm{H} 19-24$ \\
\hline \multirow{2}{*}{ edad } & $0.0365^{* * *}$ & $0.0464^{* * *}$ & $0.1466^{* * *}$ & $0.1000^{* * *}$ \\
\multirow{2}{*}{ edad2 } & $(0.001)$ & $(0.001)$ & $(0.025)$ & $(0.015)$ \\
& $-0.0005^{* * *}$ & $-0.0006^{* * *}$ & $-0.0026^{* * *}$ & $-0.0018^{* * *}$ \\
sin_edu & $(0.000)$ & $(0.000)$ & $(0.001)$ & $(0.000)$ \\
& $-0.2575^{* * *}$ & $-0.1240^{* * *}$ & $-0.3786^{* * *}$ & $-0.3229^{* * *}$ \\
primaria & $(0.006)$ & $(0.005)$ & $(0.012)$ & $(0.011)$ \\
& $-0.0814^{* * *}$ & $-0.0944^{* * *}$ & $-0.1898^{* * *}$ & $0.0135^{* * *}$ \\
primaria_comp & $(0.004)$ & $(0.003)$ & $(0.008)$ & $(0.005)$ \\
& $-0.0595^{* * *}$ & $-0.0750^{* * *}$ & $-0.1356^{* * *}$ & $0.0276^{* * *}$ \\
Secundaria & $(0.003)$ & $(0.003)$ & $(0.006)$ & $(0.004)$ \\
& $-0.0410^{* * *}$ & $-0.0559^{* * *}$ & $-0.0971^{* * *}$ & $0.0088^{* * *}$ \\
terciaria & $(0.003)$ & $(0.003)$ & $(0.004)$ & $(0.003)$ \\
& $0.0560^{* * *}$ & $0.1320^{* * *}$ & $0.0236^{* * *}$ & $-0.0151^{* * *}$ \\
terciaria_comp & $(0.002)$ & $(0.003)$ & $(0.004)$ & $(0.003)$ \\
& $0.1119^{* * *}$ & $0.2322^{* * *}$ & $0.1691^{* * *}$ & $0.0299^{* * *}$ \\
& $(0.002)$ & $(0.003)$ & $(0.007)$ & $(0.005)$
\end{tabular}

6 La estimación del modelo para el grupo $\mathrm{M}<19$, sin la variable edad2, arroja un efecto marginal para la variable edad con valor 0,033 y significativo al $99 \%$ de confianza. 


\begin{tabular}{|c|c|c|c|c|}
\hline & (1) MS 25-59 & (2) MS 25-59 & (3) M 19-24 & (4) H 19-24 \\
\hline \multirow{2}{*}{ post_terciaria } & $0.1286^{* * *}$ & $0.2972^{* * *}$ & $0.1959^{* * *}$ & $0.0492^{* * *}$ \\
\hline & $(0.002)$ & (0.003) & $(0.016)$ & $(0.008)$ \\
\hline \multirow{2}{*}{ estudia } & $-0.1041^{* * *}$ & $0.0183^{* * *}$ & $-0.3239 * * *$ & $-0.3379 * * *$ \\
\hline & $(0.004)$ & $(0.005)$ & $(0.004)$ & $(0.004)$ \\
\hline \multirow{2}{*}{ 1_menor_16 } & $0.0188^{* * *}$ & $-0.0066^{* * *}$ & & \\
\hline & $(0.002)$ & $(0.002)$ & & \\
\hline \multirow{2}{*}{ 2_menores_16 } & $0.0143^{* * *}$ & $-0.0381^{* * *}$ & & \\
\hline & $(0.002)$ & $(0.003)$ & & \\
\hline \multirow{2}{*}{ 3_menores_16 } & $0.0069^{* *}$ & $-0.0526^{* * *}$ & & \\
\hline & (0.003) & $(0.003)$ & & \\
\hline \multirow{2}{*}{ 4_menores_16 } & $0.0107^{* *}$ & $-0.0672^{* * *}$ & & \\
\hline & $(0.005)$ & $(0.005)$ & & \\
\hline \multirow{2}{*}{ 5_menores_16 } & $-0.0133^{*}$ & $-0.0935^{* * *}$ & & \\
\hline & $(0.008)$ & $(0.008)$ & & \\
\hline \multirow{2}{*}{ 6_menores_16 } & 0.0030 & $-0.1055^{* * *}$ & & \\
\hline & $(0.010)$ & $(0.010)$ & & \\
\hline \multirow{2}{*}{ menores_6_años } & & & $-0.0345^{* * *}$ & $0.0084^{* * *}$ \\
\hline & & & $(0.004)$ & $(0.003)$ \\
\hline \multirow{2}{*}{ casado } & & & $-0.2445^{* * *}$ & $0.1070^{* * *}$ \\
\hline & & & $(0.003)$ & $(0.002)$ \\
\hline \multirow{2}{*}{ mayoresnop } & $-0.0375^{* * *}$ & $-0.0313^{* * *}$ & $-0.0232^{* * *}$ & $-0.0257^{* * *}$ \\
\hline & $(0.001)$ & $(0.003)$ & $(0.004)$ & $(0.002)$ \\
\hline \multirow{2}{*}{ desemp_h } & $0.0047^{* * *}$ & $0.0212^{* * *}$ & $0.0366^{* * *}$ & $0.0059^{* * *}$ \\
\hline & $(0.002)$ & $(0.002)$ & $(0.003)$ & $(0.002)$ \\
\hline \multirow{2}{*}{ urbano } & $0.0687^{* * *}$ & $0.1311^{* * *}$ & $0.1415^{* * *}$ & $-0.0421^{* * *}$ \\
\hline & $(0.003)$ & $(0.003)$ & $(0.005)$ & $(0.003)$ \\
\hline \multirow{2}{*}{ log_ing_res_h $h$} & $-0.0060^{* * *}$ & $-0.0049^{* * *}$ & $-0.0035^{* * *}$ & $-0.0023^{* * *}$ \\
\hline & $(0.000)$ & $(0.000)$ & $(0.000)$ & $(0.000)$ \\
\hline$N$ & 758,552 & $1,140,464$ & 463,030 & 401,806 \\
\hline Pseudo R2 & 0.131 & 0.0802 & 0.117 & 0.284 \\
\hline
\end{tabular}

Errores estándar robustos entre paréntesis.

${ }^{* * *} \mathrm{p}<0.01,{ }^{* *} \mathrm{p}<0.05,{ }^{*} \mathrm{p}<0.1$

Todos los modelos incluyen variables dummy para cada año de la muestra (ref: 2013)

Fuente: Fuente: Gran Encuesta Integrada de Hogares. 
Tabla 5: Determinantes de la participación laboral -cont.- (Total Nacional). Variable dependiente: participación.

\begin{tabular}{|c|c|c|c|c|}
\hline & (5) $\mathrm{M}<19$ & (6) $\mathrm{H}<19$ & (7) $M>59$ & (8) $\mathrm{H}>59$ \\
\hline \multirow{2}{*}{ edad } & $-0.0497^{* * *}$ & $0.0273^{* * *}$ & $-0.0104^{* * *}$ & -0.0053 \\
\hline & $(0.007)$ & $(0.008)$ & $(0.003)$ & $(0.005)$ \\
\hline \multirow{2}{*}{ edad2 } & $0.0028^{* * *}$ & $0.0012^{* * *}$ & -0.0000 & $-0.0002^{* * *}$ \\
\hline & $(0.000)$ & $(0.000)$ & $(0.000)$ & $(0.000)$ \\
\hline \multirow{2}{*}{ sin_edu } & $-0.0801^{* * *}$ & $-0.0210^{* *}$ & 0.0033 & -0.0077 \\
\hline & $(0.004)$ & $(0.008)$ & $(0.005)$ & $(0.008)$ \\
\hline \multirow{2}{*}{ primaria } & $-0.0091^{* *}$ & $0.1537^{* * *}$ & $0.0073^{*}$ & $0.0362^{* * *}$ \\
\hline & $(0.004)$ & $(0.007)$ & $(0.004)$ & $(0.007)$ \\
\hline \multirow{2}{*}{ primaria_comp } & -0.0013 & $0.1488^{* * *}$ & -0.0012 & $0.0218^{* * *}$ \\
\hline & $(0.004)$ & $(0.006)$ & $(0.004)$ & $(0.007)$ \\
\hline \multirow{2}{*}{ Secundaria } & $-0.0240^{* * *}$ & $0.0700^{* * *}$ & 0.0040 & $0.0139^{*}$ \\
\hline & (0.003) & $(0.004)$ & $(0.005)$ & $(0.008)$ \\
\hline \multirow{2}{*}{ terciaria } & $0.0270^{* * *}$ & $0.0865^{* * *}$ & $0.0384^{* * *}$ & $0.0402^{* * *}$ \\
\hline & $(0.005)$ & $(0.009)$ & $(0.008)$ & $(0.011)$ \\
\hline \multirow{2}{*}{ terciaria_comp } & $0.4579^{* * *}$ & $0.3439^{* *}$ & $0.0689^{* * *}$ & $0.0734^{* * *}$ \\
\hline & $(0.111)$ & $(0.169)$ & $(0.009)$ & $(0.009)$ \\
\hline \multirow{2}{*}{ post_terciaria } & -0.0294 & $-0.1489 * * *$ & $0.1547^{* * *}$ & $0.1849^{* * *}$ \\
\hline & $(0.063)$ & $(0.048)$ & $(0.012)$ & $(0.010)$ \\
\hline \multirow{2}{*}{ estudia } & $-0.2166^{* * *}$ & $-0.4279^{* * *}$ & & \\
\hline & $(0.004)$ & $(0.004)$ & & \\
\hline \multirow{2}{*}{ 1_menor_16 } & & & $-0.0070^{* *}$ & $0.0116^{* * *}$ \\
\hline & & & $(0.003)$ & $(0.005)$ \\
\hline \multirow{2}{*}{ 2_menores_16 } & & & $-0.0152^{* * *}$ & 0.0079 \\
\hline & & & $(0.004)$ & $(0.006)$ \\
\hline \multirow{2}{*}{ 3_menores_16 } & & & -0.0074 & $0.0257^{* * *}$ \\
\hline & & & $(0.005)$ & $(0.009)$ \\
\hline \multirow{2}{*}{ 4_menores_16 } & & & 0.0014 & $0.0231^{*}$ \\
\hline & & & (0.009) & $(0.013)$ \\
\hline \multirow{2}{*}{ 5_menores_16 } & & & 0.0238 & $0.0475^{* *}$ \\
\hline & & & $(0.016)$ & $(0.022)$ \\
\hline \multirow{2}{*}{ 6_menores_16 } & & & 0.0052 & $0.0597^{* *}$ \\
\hline & & & $(0.016)$ & $(0.025)$ \\
\hline
\end{tabular}




\begin{tabular}{|c|c|c|c|c|}
\hline & (5) $\mathrm{M}<19$ & (6) $\mathrm{H}<19$ & (7) $M>59$ & (8) $\mathrm{H}>59$ \\
\hline \multirow{2}{*}{ menores_6_años } & $0.0077^{* * *}$ & $0.0288^{* * *}$ & & \\
\hline & $(0.002)$ & (0.003) & & \\
\hline \multirow{2}{*}{ casado } & $-0.0771^{* * *}$ & $0.2367^{* * *}$ & $-0.0416^{* * *}$ & $0.0619^{* * *}$ \\
\hline & $(0.002)$ & $(0.016)$ & $(0.002)$ & $(0.004)$ \\
\hline \multirow{2}{*}{ pensionado } & & & $-0.1027^{* * *}$ & $-0.3726^{* * *}$ \\
\hline & & & $(0.002)$ & $(0.004)$ \\
\hline \multirow{2}{*}{ mayoresnop } & $-0.0203^{* * *}$ & $-0.0451^{* * *}$ & & \\
\hline & $(0.003)$ & $(0.003)$ & & \\
\hline \multirow{2}{*}{ desemp_h } & $-0.0050^{* * *}$ & $-0.0194^{* * *}$ & $-0.0235^{* * *}$ & $-0.0269^{* * *}$ \\
\hline & $(0.002)$ & $(0.002)$ & $(0.002)$ & $(0.004)$ \\
\hline \multirow{2}{*}{ urbano } & -0.0009 & $-0.1544^{* * *}$ & $-0.0307^{* * *}$ & $-0.1916^{* * *}$ \\
\hline & $(0.002)$ & $(0.003)$ & $(0.003)$ & $(0.004)$ \\
\hline \multirow{2}{*}{ log_ing_res_h } & $-0.0005^{*}$ & $-0.0021^{* * *}$ & $-0.0057^{* * *}$ & $-0.0069^{* * *}$ \\
\hline & $(0.000)$ & $(0.000)$ & $(0.000)$ & $(0.000)$ \\
\hline$N$ & 575,964 & 574,468 & 461,398 & 357,440 \\
\hline Pseudo R2 & 0.181 & 0.290 & 0.108 & 0.225 \\
\hline
\end{tabular}

Errores estándar robustos entre paréntesis.

*** $\mathrm{p}<0.01,{ }^{* *} \mathrm{p}<0.05,{ }^{*} \mathrm{p}<0.1$

Todos los modelos incluyen variables dummy para cada año de la muestra (ref: 2013)

Fuente: Gran Encuesta Integrada de Hogares.

La aceleración se observa también en el caso de los hombres, para los cuales el efecto marginal estimado de edad tiene valor positivo, tal y como se esperaba (modelos 5 y 6, tabla 5).

Para todas las mujeres menores de 59 años, sin importar si son no casadas o casadas, entre más alto sea el nivel educativo alcanzado, existe mayor probabilidad de participación. Los efectos marginales negativos y estadísticamente significativos de los niveles anteriores a la secundaria completa indican que, al haber alcanzado solo estos niveles, la probabilidad de participación es menor respecto de la de aquellas que alcanzaron dicho nivel de referencia; mientras que los efectos marginales positivos y estadísticamente significativos de los niveles posteriores a secundaria completa indican lo contrario. En el caso de las mujeres casadas de 25 a 59 años, el efecto positivo que tiene alcanzar un nivel por encima de la secundaria sobre la probabilidad de participación, es más del doble que aquel para las no casadas (modelos 1 y 2). 
El efecto de la educación superior es muy importante para los individuos menores de 25 años de edad, especialmente para las mujeres, pues el efecto marginal de alcanzar estos niveles puede ser más de cuatro veces superior al de los hombres ${ }^{7}$. En el caso de estos últimos, sin embargo, alcanzar niveles educativos inferiores a la secundaria completa tiene un efecto positivo y significativo en la probabilidad de participar, y dicho efecto es decreciente a partir del menor nivel que es la primaria incompleta, lo que implica que individuos que alcanzaron los menores niveles de educación tienen mayor probabilidad de participar que aquellos que cuentan con niveles cercanos a la secundaria completa e, inclusive, frente a quienes han alcanzado terciaria incompleta. En este sentido, si tan solo se han alcanzado niveles inferiores de educación, se espera que, en promedio, la edad de ingreso al mercado laboral sea menor para los hombres que para las mujeres, pues el costo marginal de ofrecer tiempo positivo en el mercado de trabajo se reduce estimulando la participación.

Para mujeres y hombres mayores de 59 años, el efecto de la educación sobre la probabilidad de participar en el mercado de trabajo es similar al observado para los hombres menores de 25 años, aunque los efectos marginales son mínimos, ya que, en promedio, estos individuos tienen menores niveles de educación que el resto de la población (modelos 7 y 8, tabla 5). En este mismo grupo etario, la probabilidad de participación se ve afectada positivamente por los niveles de educación superiores a la secundaria completa, en el caso de hombres y mujeres, lo cual muestra un claro efecto poblacional, pues, en promedio, los individuos más jóvenes de este grupo pudieron alcanzar niveles de educación más altos que aquellos con esta edad diez años atrás.

Con excepción de las mujeres casadas, estar estudiando, en la actualidad, incrementa el costo marginal de ofrecer tiempo positivo en el mercado de trabajo y reduce la probabilidad de participación. Como es de esperarse, este efecto es más fuerte y determinante en los individuos jóvenes menores de 25 años, para los cuales no estudiar o estudiar, en la actualidad, establece, en promedio, si estos individuos participan o no en el mercado de trabajo, pues las diferencias en la probabilidad de participar están entre 22 y 43 puntos porcentuales en el caso de los menores de 19 años (modelos 5 y 6, tabla 5), y son mayores a 30 puntos en el caso de mujeres y hombres con edades entre 19 y 24 años (modelos 3 y 4, tabla 4). Por su parte, la decisión de participar y estudiar está relacionada positivamente en el caso de las mujeres casadas entre los 25 y 59 años (modelo 2), aunque el efecto marginal es diez veces menor (en valor absoluto) al estimado para las mujeres no casadas.

7 Comparación de los efectos de niveles de educación superior completa en los modelos 3 y 4 , tabla 4 . 
Para las mujeres en el grupo de 25 a 59 años, la presencia de menores de 16 años en el hogar tiene efectos marginales contrarios al diferenciar entre no casadas y casadas. Para las primeras, la dependencia económica reduce el costo marginal del tiempo e incrementa la probabilidad de participación, mientras que para las casadas, al contar con un apoyo económico de su cónyuge, la presencia de menores y su número aumenta el costo en el margen del tiempo ante una dependencia no económica y la posibilidad de tomar decisiones conjuntas en el hogar (Chiappori, 1992). Al igual que para este último grupo de mujeres, la presencia de uno y / o dos menores de 16 años tiene un efecto negativo en la probabilidad de participar de las mujeres mayores de 59 años, mientras que en el caso de los hombres del mismo grupo de edad, la similitud se da con las mujeres solteras y su situación de responsabilidad económica en el hogar.

Los efectos de la presencia de menores en la probabilidad de participación de hombres y mujeres de 19 a 24 años de edad van también en dirección contraria. El efecto marginal es negativo y significativo para las mujeres, y positivo y significativo en el caso de los hombres (modelos 3 y 4, tabla 4). Ante la presencia de menores de 6 años, se incrementa la probabilidad de participación de los hombres en este rango de edad, pues el costo marginal de ofrecer tiempo positivo en el mercado de trabajo se reduce al crearse una dependencia económica, mientras que para las mujeres sucede lo contrario ante la existencia de una dependencia no económica que incrementa dicho costo. En el grupo de individuos más jóvenes, sin embargo, no se presenta esta relación complementaria, pues el valor del efecto marginal es positivo y estadísticamente significativo en ambos casos, lo cual implica que para estos hombres y mujeres es la dependencia económica la que domina y tiene influencia sobre el costo marginal ${ }^{8}$.

Como se esperaba, la presencia de mayores no participantes reduce la probabilidad de participación para hombres y mujeres menores de 59 años. Además, para todos los grupos de edad establecidos en el análisis, el mayor nivel de ingreso del resto de individuos que conforman el hogar incrementa el costo marginal del tiempo y reduce la probabilidad de participación del individuo. En este caso, el efecto marginal puede entenderse como una semi-elasticidad $\mathrm{y}$, ante un cambio en el 10\% del ingreso del resto del hogar, la probabilidad de participación se reduciría en un máximo de siete puntos porcentuales, en el

8 A menos que el individuo sea caracterizado como jefe de hogar, en la ECH y en la GEIH no es posible identificar perfectamente la relación parental; de allí que no se hable de la presencia de hijos sino de la presencia de menores. En este caso particular, es probable que en el grupo de menores de 19 años haya menos mujeres que sean madres, al hacer la comparación con el grupo de mujeres con edades entre los 19 a 24 años, por lo que la dependencia no económica puede tener una relevancia significativamente menor para el primer grupo. 
caso de los hombres mayores de 59 años, y en un mínimo de 0,5 puntos porcentuales en el caso de las mujeres menores de 19 años de edad.

Un hallazgo importante para los determinantes de la participación de hombres y mujeres mayores de 59 años, es el efecto que tiene contar con un ingreso por pensión. El efecto marginal es negativo y significativo, señalando que, al no percibir un ingreso por pensión, la probabilidad de participación se incrementa en gran medida. Otro efecto poblacional puede determinar la importancia de este efecto, ya que, a medida que la población envejece, la importancia de la pensión sobre la participación de estos individuos es crítica, pues determina su decisión de retiro del mercado de trabajo. En este caso, la probabilidad de participación va a estar asociada con la fortaleza del sistema de seguridad social en pensiones y su capacidad de cubrimiento del riesgo de la vejez. Como lo señalan Guataquí, et al. (2009), ante la alta evasión del sistema de seguridad social formal, los mecanismos informales de ahorro para la vejez, como lo son la acumulación de capital humano de los hijos y la adquisición de otros activos, se hacen claramente insuficientes, dada la baja propensión marginal al ahorro en una economía donde la informalidad laboral y empresarial son preponderantes.

Por su parte, el número de desempleados en el hogar tiene un efecto positivo y significativo en la probabilidad de participación de las mujeres entre 19 y 59 años. En este sentido, si esta variable representa la situación del mercado laboral que enfrentan los individuos, el mecanismo de transmisión es el del workeradded effect o trabajador adicional: la percepción de una mala/buena situación del mercado de trabajo impulsa/reduce la probabilidad de participar ante la/el reducción/incremento del ingreso laboral esperado del hogar. Para los individuos menores de 19 años, los hombres entre 19 y 24 años, y mujeres y hombres mayores de 59 años, el efecto marginal estimado es estadísticamente significativo y tiene signo negativo. Es posible asociar este resultado con lo que se definió como el discouraged-worker effect o trabajador desalentado: la percepción de una mala/buena situación del mercado de trabajo, reduce/incrementa la probabilidad de participar ante una/un reducción/incremento en la probabilidad de conseguir empleo en el mercado de trabajo. Aunque requeriría un análisis más profundo que va más allá de los alcances de este documento, una primera hipótesis que podría dar explicación a estas diferencias radicaría en la posibilidad de que durante el periodo pos crisis, el efecto sustitución intrafamiliar laboral se haya hecho significativamente menor para las personas muy jóvenes y los adultos mayores, de acuerdo con los fundamentos que expone López (1985)9 .

9 Ver nota al pie número 5. 
Cómo se señaló en la sección 0, para los grupos etarios en los que se distinguió por género, era importante introducir una variable que determinara el efecto de su situación conyugal, aunque puede haber endogeneidad implícita al suponer que dicha situación es exógena a la decisión de participación (Heckman, 1978) ${ }^{10}$. El resultado obtenido es estándar, pues la mujer casada, ya sea joven o mayor, tiene una menor probabilidad de participar, mientras que para el hombre casado se espera lo contrario.

Por último, el efecto marginal de la variable urbano es negativo para los hombres entre 19 y 24 años (modelo 4, tabla 4), para hombres y mujeres menores de 19 años (modelos 5 y 6, tabla 5) y para hombres y mujeres mayores de 59 años (modelos 7 y 8, tabla 5). En el caso de los dos primeros grupos, este resultado tiene relación con el hecho de que los hombres jóvenes pueden ingresar de manera más fácil al mercado de trabajo mediante la realización de labores agrícolas, mientras que para el segundo grupo es posible que los individuos situados en zonas rurales permanezcan más tiempo en el mercado. Por otro lado, y como era de esperarse, para las mujeres menores de 59 años la probabilidad de participar es mayor en el caso de aquellas cuya ubicación geográfica está en ciudades o centros poblados. Este es un resultado que se repite en los trabajos de determinantes de la participación en Colombia.

\section{Perfiles de la participación laboral}

A partir de los resultados de los modelos de participación estimados, se propone un análisis adicional para determinar el aporte de los determinantes de la participación durante el periodo de estudio. La idea es construir perfiles de participación para grupos de individuos específicos a partir de las probabilidades estimadas de participación. El perfil muestra cómo estas probabilidades han evolucionado a lo largo del tiempo y permite establecer razones por las cuales se presentan dichos cambios a partir de comparaciones asociadas a factores como la presencia de menores en el hogar, el nivel educativo, el estado civil, el número de desempleados en el hogar o, en el caso particular de los individuos de 60 años o más, el cubrimiento de la pensión.

En el grupo de personas entre 25 y 59 años de edad, se construyó el perfil para las mujeres de 25 a 35 años, mientras que para el grupo de individuos con edades menores de 19 años, y el de aquellos entre 19 y 24 años, se establecieron

10 A pesar de separar entre no casadas y casadas, este mismo riesgo se corre en los modelos estimados para las mujeres entre 25 y 59 años. 
los perfiles para las/los mujeres/hombres de 15 a 18 años y las/los mujeres/ hombres de 20 a 23 años. Finalmente, para aquellos con 60 años o más, el perfil construido fue para las/los mujeres/hombres de 60 a 65 años. Aunque arbitraria, la elección de estos perfiles buscó que, con relación al grupo etario correspondiente, las tasas de participación fueran altas, con el fin de asociar el análisis con el incremento observado de las tasas de participación durante el periodo. En el caso de las/los mujeres/hombres de 15 a 18 años, esta observación puede no cumplirse, pero es interesante analizar el comportamiento de quienes, posiblemente, se enfrentan por primera vez a la decisión de extender la oferta de trabajo.

De acuerdo con el grupo de edad correspondiente, se estima el modelo de determinantes de participación expresado en la ecuación (3) en cada uno de los periodos $t=\{2002, \ldots, 2005,2007, \ldots, 2013\}$ y, para cada uno de los individuos $i$ que conforman dicho grupo $k$, se determinan luego las probabilidades puntuales condicionadas de participación:

$$
\begin{aligned}
& P P_{i, t \mid \bar{x}_{j}}=P\left(y_{i, t}^{*}=1 \mid x_{j, i}=\bar{x}_{j}\right)=\Psi\left(x_{i, t\left(x_{t, i} \neq x_{j, i}\right)}^{\prime} \hat{\beta}_{\left(\beta_{\ell} \neq \beta_{j}\right)}+\hat{\beta}_{j} \bar{x}_{j}+z_{i, t}^{\prime} \hat{\delta}\right) \\
& P P_{i, t \mid \bar{z}_{m}}=P\left(y_{i, t}^{*}=1 \mid z_{m, i}=\bar{z}_{m}\right)=\Psi\left(x_{i, t}^{\prime} \hat{\beta}+z_{i, t\left(z_{n, i} \neq z_{m, i}\right)}^{\prime} \hat{\delta}_{\left(\delta_{n} \neq \delta_{m}\right)}+\hat{\delta}_{m} \bar{z}_{m}\right)
\end{aligned}
$$

Las probabilidades estimadas están condicionadas a algún atributo $x_{j}$ que afecta el beneficio marginal, o algún atributo $z_{m}$ determinante del costo marginal de ofrecer tiempo positivo en el mercado de trabajo. Para cada una las probabilidades puntuales calculadas a partir de la ecuación (4), se calcula un intervalo de confianza del $90 \%$ de acuerdo con:

$$
\begin{aligned}
& {\left[P P_{i, t \mid \bar{x}_{j}}^{u p}, P P_{i, t \mid \bar{x}_{j}}^{l o}\right]=P P_{i, t \mid \bar{x}_{j}} \pm 1.96 \times \sigma_{i, t \mid \bar{x}_{j}}} \\
& {\left[P P_{i, t \mid \bar{z}_{m}}^{u p}, P P_{i, t \mid \bar{z}_{m}}^{l o}\right]=P P_{i, t \mid \bar{z}_{m}} \pm 1.96 \times \sigma_{i, t \mid \bar{z}_{m}}}
\end{aligned}
$$

Donde $\sigma_{i, t \mid}$. es el error estándar del operador lineal de la función $\Psi(\cdot)$. El cálculo de los intervalos de confianza permite determinar si las diferencias entre las probabilidades de participación estimadas son estadísticamente significativas. El perfil condicionado de participación $\left\{P P_{s \mid \cdot}^{l o, k}, P P_{s \mid}^{k}, P P_{s \mid}^{u p, k}\right\}_{s \in t}$ para el grupo $k$ se define a partir de: 


$$
\begin{aligned}
& P P_{s \mid \cdot}^{l o, k}=\frac{1}{N_{k}} \sum_{i \in k} P P_{i, s \mid}^{l o} \\
& P P_{s \mid \cdot}^{k}=\frac{1}{N_{k}} \sum_{i \in} P P_{i, s \mid \cdot} \\
& P P_{s \mid \cdot}^{u p, k}=\frac{1}{N_{k}} \sum_{i \in k} P P_{i, s \mid \cdot}^{u p}
\end{aligned}
$$

\subsection{Mujeres de 25 a 35 años}

El perfil condicionado de la probabilidad de participación, establecido por las ecuaciones (4) y (5), para las mujeres de 25 a 35 años, se puede observar en la figura 3. Como era de esperarse, a partir del análisis de determinantes, a lo largo del periodo de estudio las mujeres no casadas tienen una probabilidad de participación en el mercado laboral significativamente mayor a la de las casadas. Dicha diferencia, sin embargo, parece diluirse si la mujer ha alcanzado niveles de educación superior.

Sin importar su estado conyugal, al condicionar por el nivel educativo alcanzado, surgen diferencias significativas en la probabilidad de participar de estas mujeres, siendo más notables en el caso de las mujeres casadas, para las cuales terminar la educación universitaria implica incrementos de casi 20 puntos porcentuales en dicha probabilidad, aunque a partir de 2008 se ha presentado un aumento significativo de la probabilidad de participar de las mujeres casadas con niveles de educación hasta la terciaria incompleta (panel a). En el caso de las mujeres no casadas, las diferencias importantes se presentan al comparar las probabilidades de participación cuando se tiene o no educación superior completa (panel b).

Condicionada a la presencia de menores, y solo para el caso de las mujeres casadas, el número de menores es un determinante significativo de las diferencias en la probabilidad de participación. Como se observa en el panel c de la figura 3, estas diferencias se han ampliado en favor de las mujeres con menor número de menores presentes en el hogar, lo que implica que, al menos en parte, la menor presencia de menores ha contribuido a las mayores tasas de participación observadas para el grupo de mujeres con edades entre 25 y 59 años después de 2007. Finalmente, y a pesar de que los efectos marginales son significativos en el modelo de determinantes, las diferencias en la probabilidad de participación de este grupo de mujeres no parecen ser explicadas por el número de desempleados en el hogar (paneles e y f). 
a) Casadas: Efecto nivel educativo

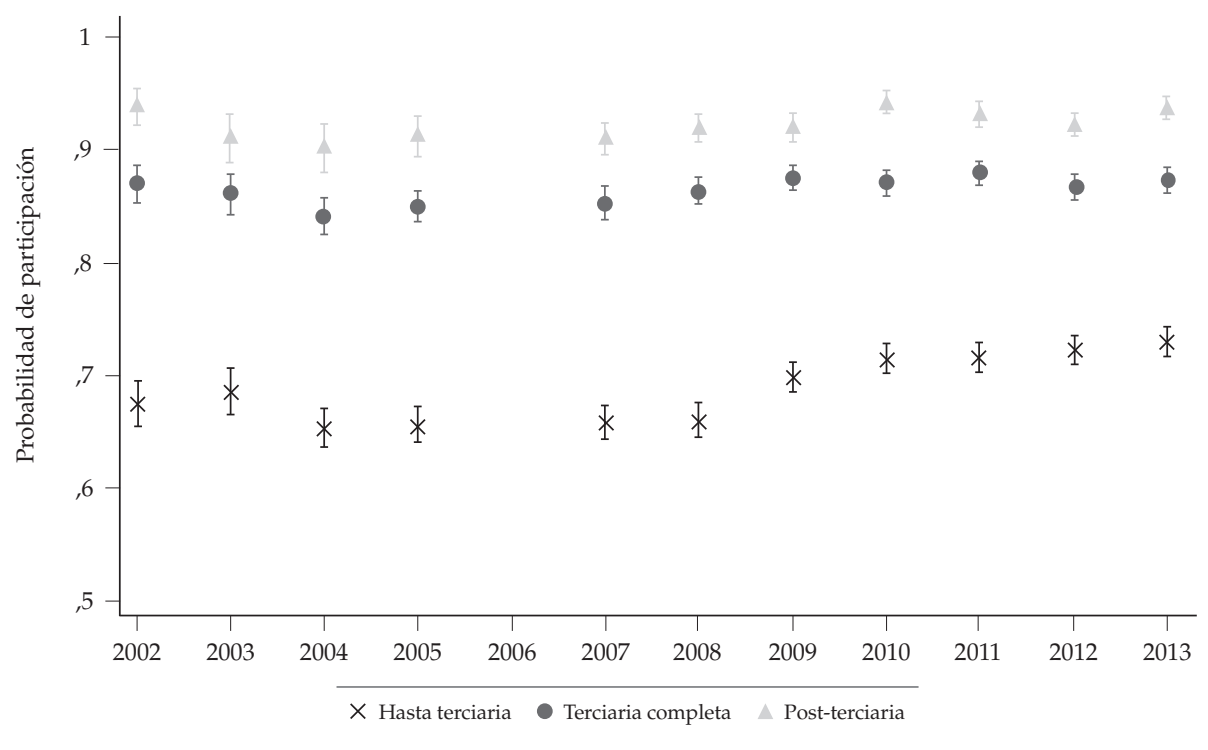

b) No casadas: Efecto nivel educativo

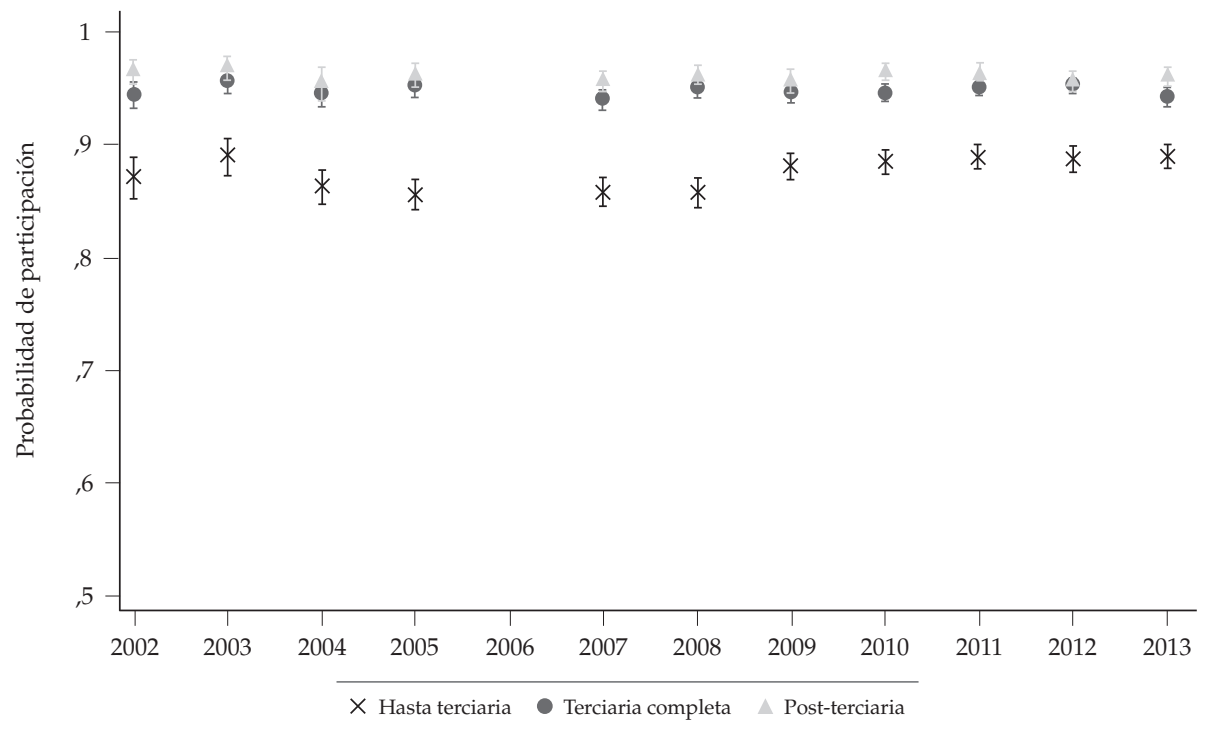


c) Casadas: Efecto menores de 16 años

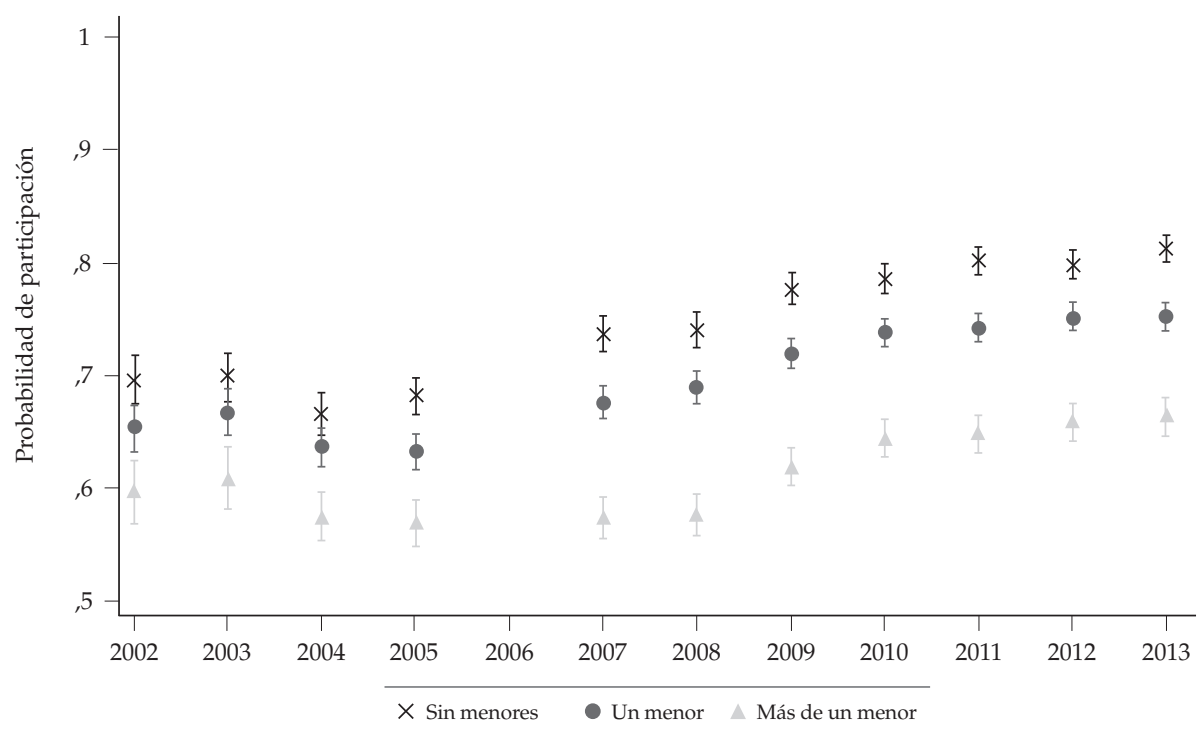

d) No casadas: Efecto menores de 16 años

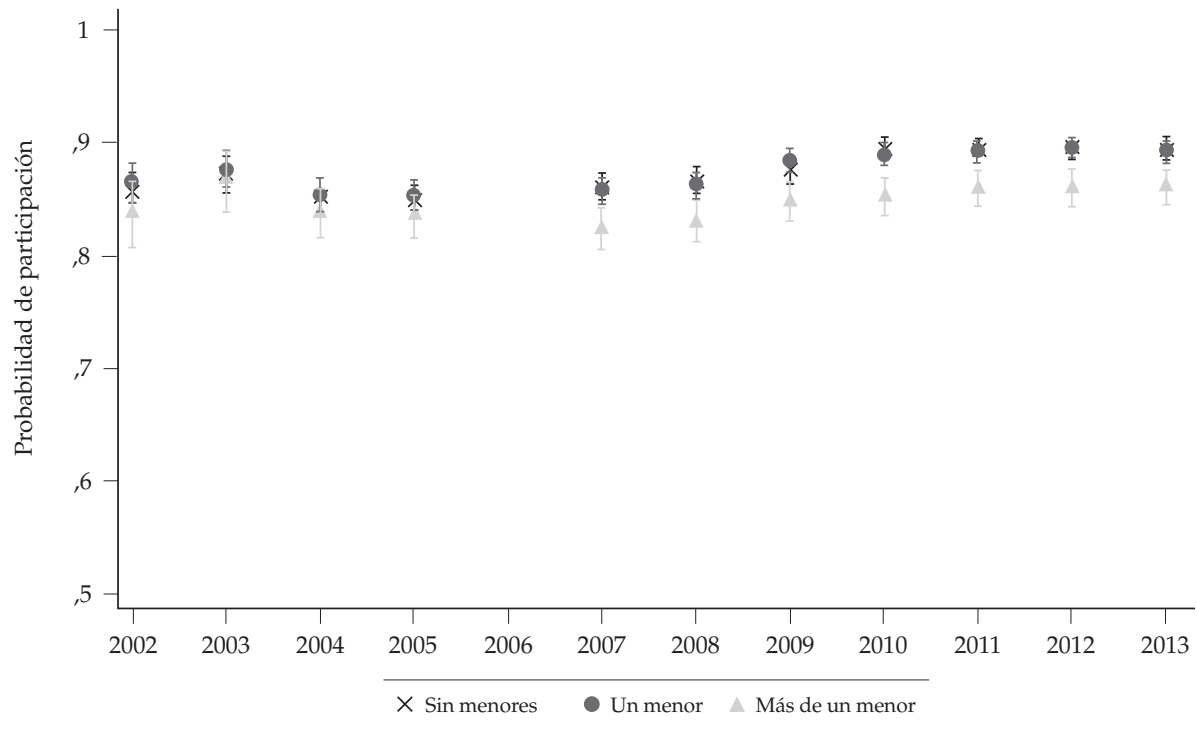

Figura 3, continúa página siguiente 
e) Casadas: Efecto número de desempleados en el hogar

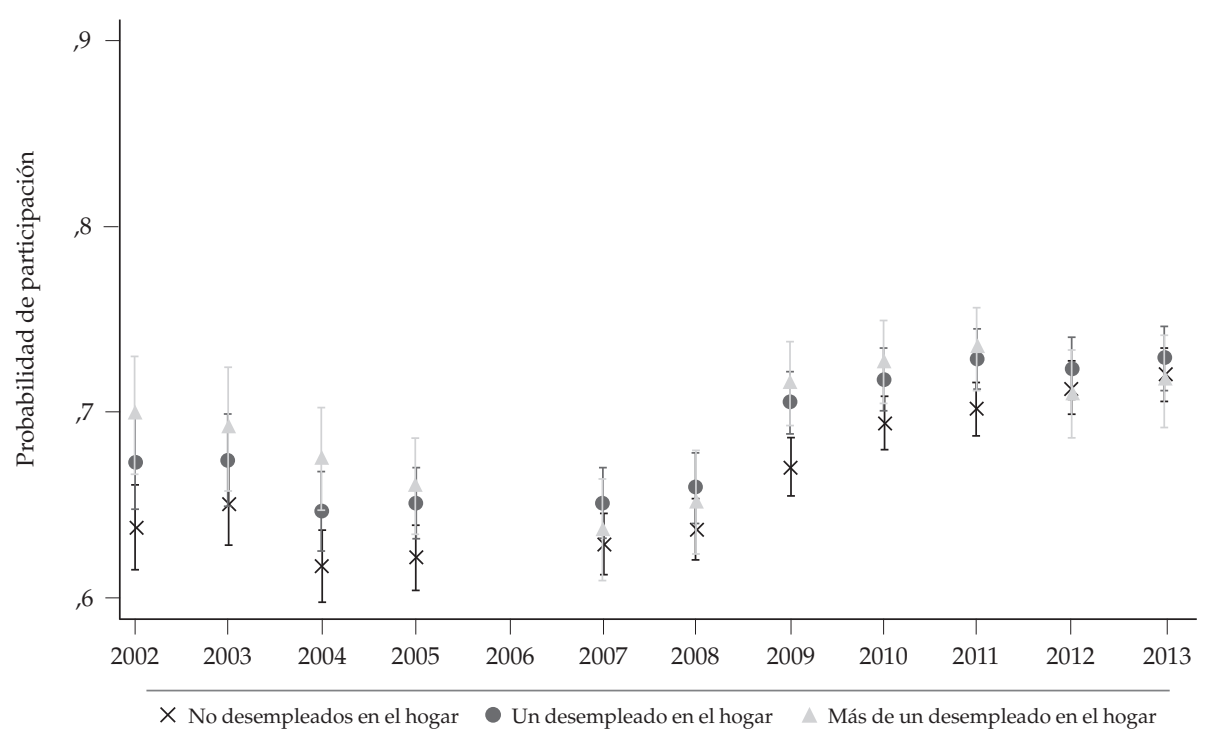

f) No casadas: Efecto número de desempleados en el hogar

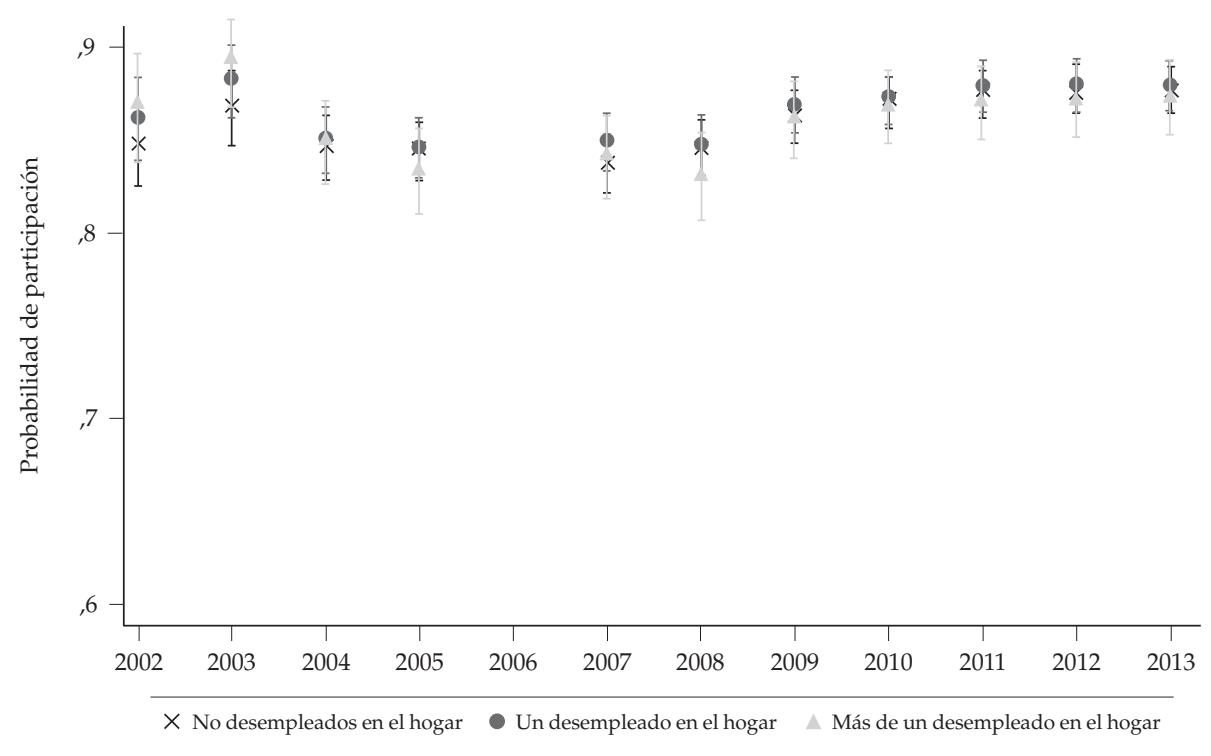

Figura 3. Probabilidad de participación promedio de las mujeres de 25 a 35 años Fuente: Estimaciones de los autores. 


\subsection{Mujeres/hombres de 15 a 18 años}

Entre los factores que parecen explicar, al menos en parte, el incremento en la tasa de participación observado para los individuos parte de este perfil se encuentran el incremento, a partir de 2009, de la probabilidad de participar condicionada a estar actualmente estudiando (paneles a y b de la figura 4), así como de la condicionada a haber alcanzado un nivel de educación terciaria incompleto (paneles e y f). Estos dos efectos parecen complementarse entre sí, pues en este grupo de edad, entre 2009 y 2013, el número de participantes que se encuentran estudiando y con educación terciaria incompleta se incrementó en un $46 \%$ al pasar de ser 34409 a 50268, de acuerdo con nuestros cálculos, a partir de los datos de la GEIH. Sin embargo, y a pesar de representar menos en valor absoluto, los individuos en este grupo de edad que participan alcanzaron niveles de terciaria incompleta; y los que no estudian, se incrementaron en un $90 \%$ en el mismo periodo, lo cual señala que, a pesar de haber cursado niveles de educación superior, es probable que el costo marginal de mantenerse por fuera del mercado de trabajo haya sido tan alto como para abandonar sus estudios.

En el caso de los hombres, la probabilidad condicionada de participar, al haber alcanzado niveles de secundaria incompleta, se incrementó también significativamente frente a las de secundaria completa y terciaria completa (panel f), pero, en este caso, el complemento entre estar estudiando hoy tal nivel de educación y la decisión de participación parece ser más fuerte, pues el número de participantes en esta situación se incrementó cerca del $18 \%$ entre 2009 y 2013, mientras que la variación de aquellos que no estudian habiendo alcanzado dicho nivel fue de apenas el $8 \%$.

Aunque no se asocia directamente con la dinámica creciente de la participación de este grupo, el perfil en el panel d de la figura 4 muestra, en el caso de los hombres, una reducción relativa del efecto de contar con al menos un menor de 6 años en el hogar frente al de no contar con alguno, lo cual puede ser evidencia de una reducción del peso de la dependencia económica en la decisión de participación. En el caso de las mujeres (panel c), no hay un cambio significativo en este sentido. 
a) Mujeres: Efecto estudia

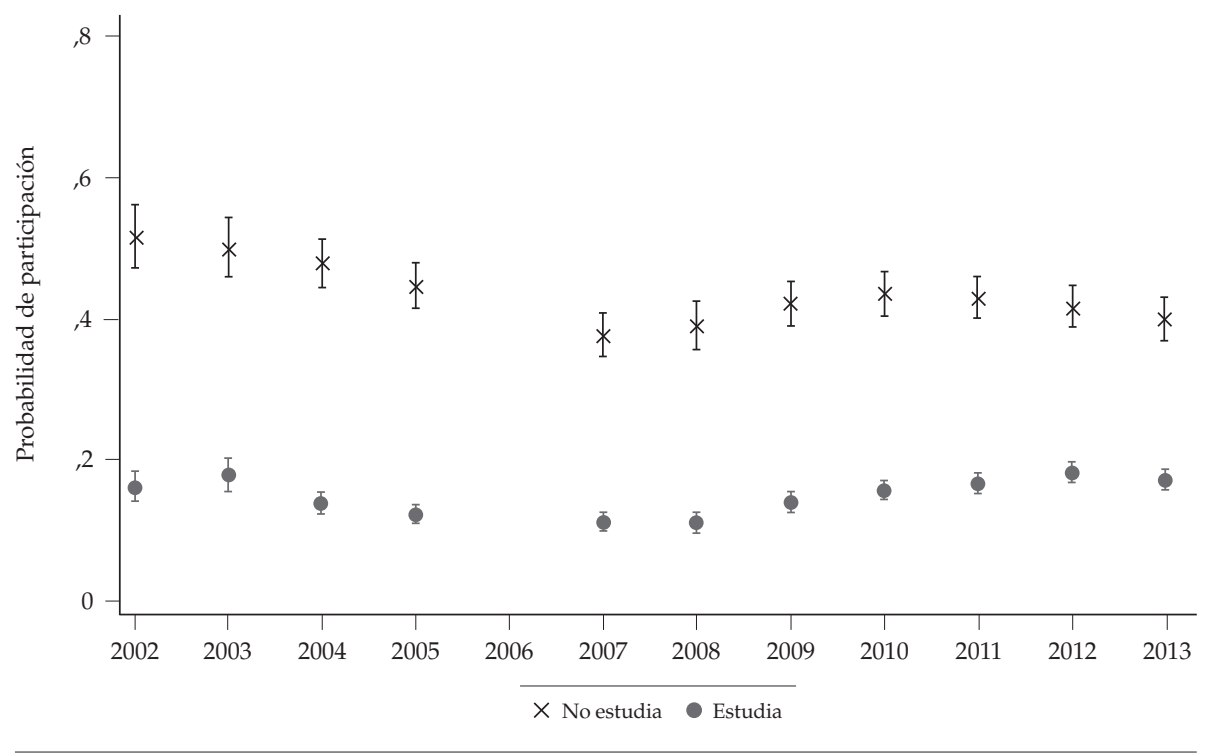

b) Hombres: Efecto estudia

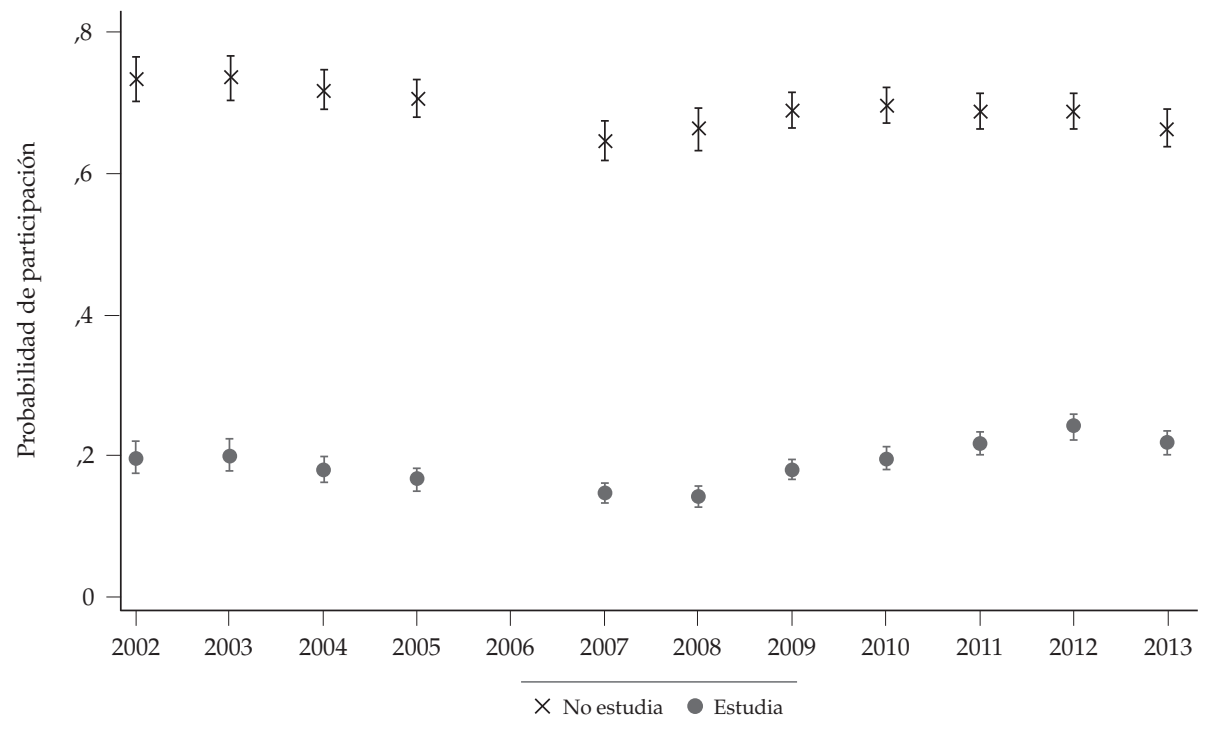


c) Mujeres: Efecto menores de 6 años

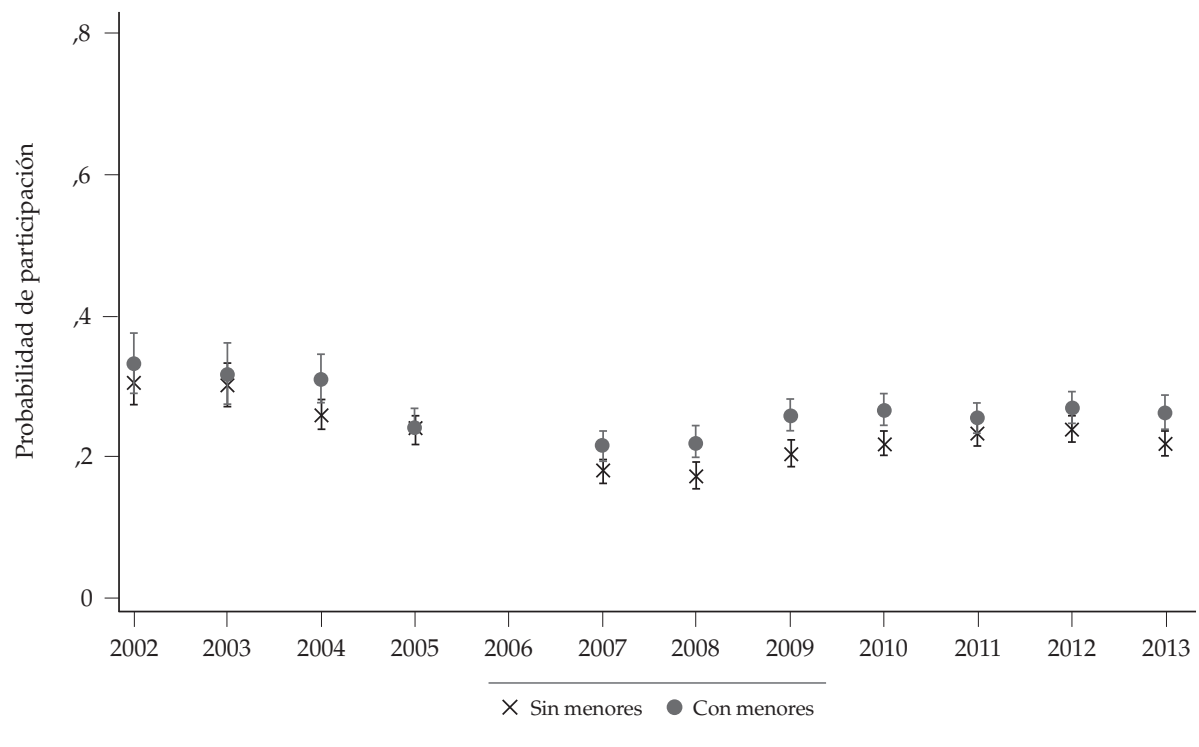

d) Hombres: Efecto menores de 6 años

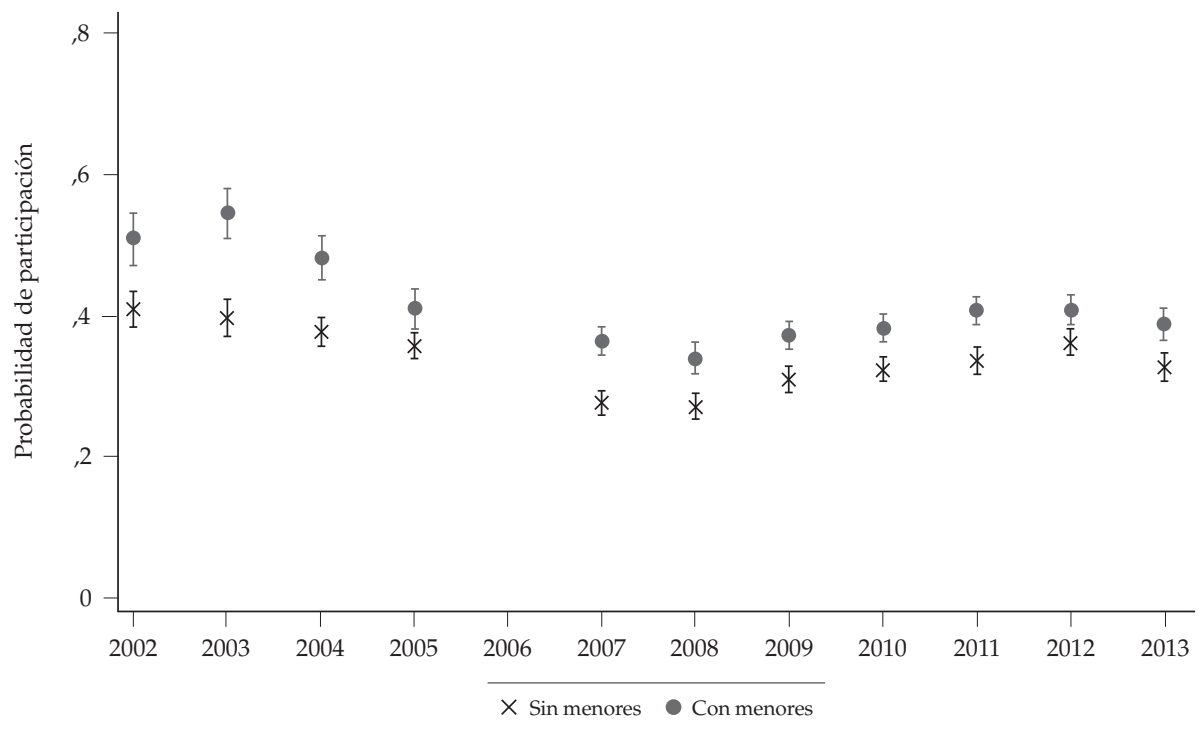

Figura 4, continúa página siguiente 


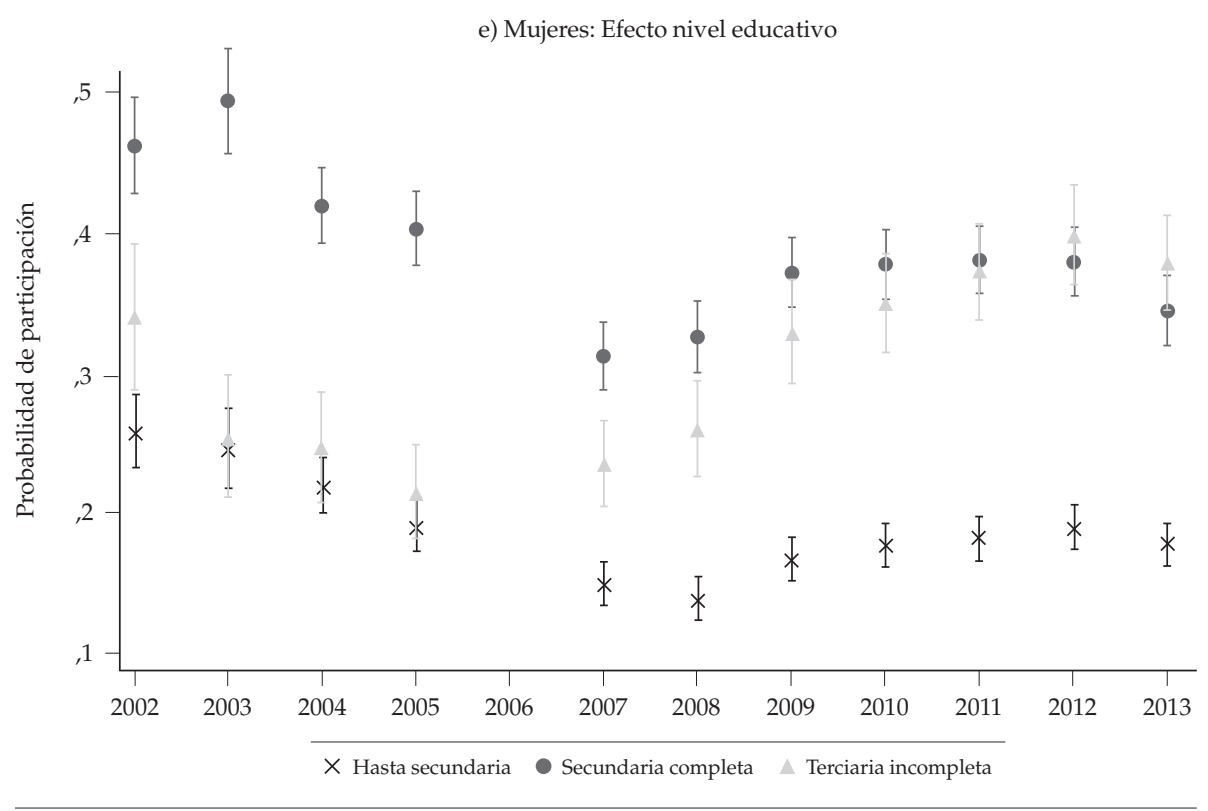

b) Hombres: Efecto estudia

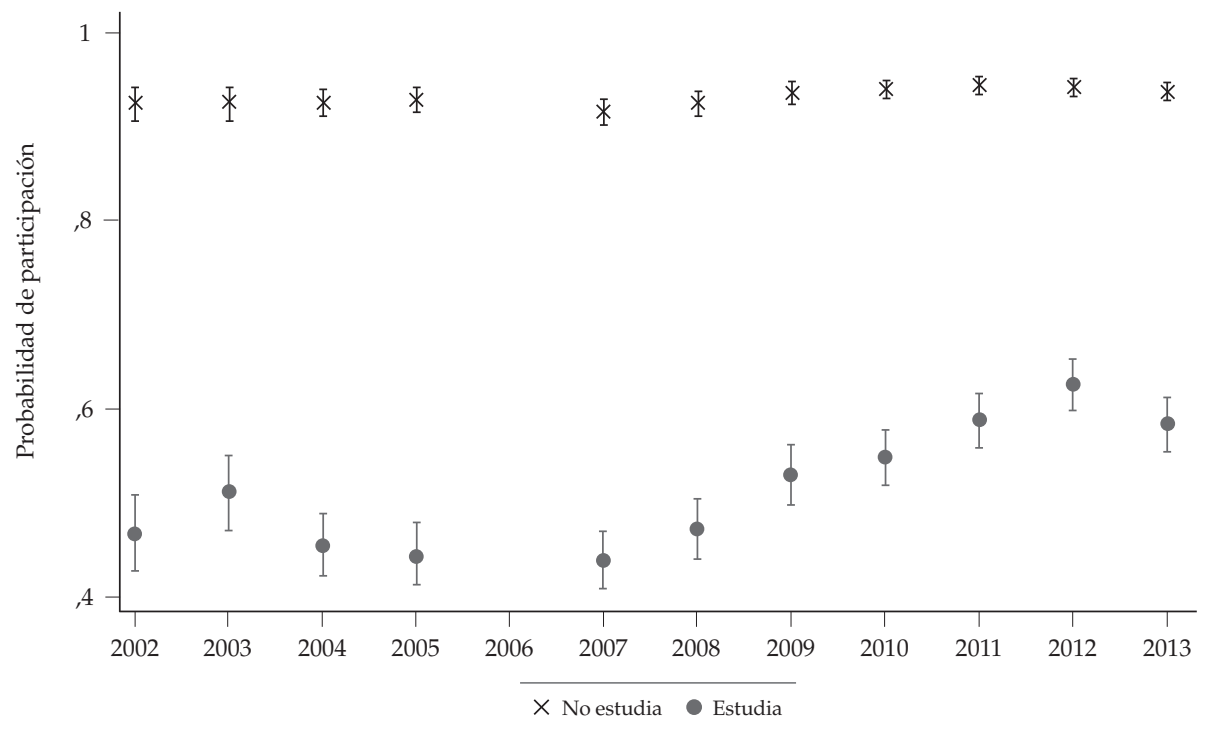

Figura 4: Probabilidad de participación promedio de mujeres y hombres de 15 a 18 años Fuente: Estimaciones de los autores. 


\subsection{Mujeres/hombres de 20 a 23 años}

$\mathrm{Al}$ igual que sucedió con las/los mujeres/hombres entre 15 y 18 años, el incremento en la participación de ambos sexos entre 20 y 23 años está altamente asociado con incrementos significativos de los efectos terciaria incompleta (paneles e y $\mathrm{f}$ de la figura 4: Probabilidad de participación promedio de mujeres y hombres de 15 a 18 años), y estudia (paneles a y b). Esto corrobora la plausibilidad de la hipótesis que señala que, para los jóvenes, en Colombia, la sustitución entre estudiar y participar ha venido reduciéndose a lo largo de los últimos 11 años, lo cual se ha relacionado con incrementos significativos en la participación en el mercado de trabajo.

Además de esto, el perfil de probabilidades condicionadas para este grupo de individuos muestra que otra fuente de incrementos en la participación de las mujeres de 20 a 23 años es la reducción relativa y significativa del efecto que tiene la presencia de menores de 6 años en el hogar (panel c), lo cual contrasta con lo observado para los hombres, en los que, por el contrario, este mismo efecto genera una tendencia creciente en la probabilidad de participación (panel d).

Por su parte, el número de desempleados en el hogar parece ser importante para explicar, en parte, la dinámica solo en el periodo 2002-2005 y únicamente para el caso de las mujeres, para las cuales las diferencias se redujeron en favor de aquellas con ninguna presencia de desempleados en el hogar (panel g). La reducción del número promedio de desempleados y la mejor situación del mercado de trabajo contribuyeron a la reducción de la tasa de participación de este grupo etario durante este subperiodo. Mientras tanto, en el periodo 2002-2005, el número de desempleados promedio por hogar para estas mujeres cayó a una tasa del 9,9\% anual.

\subsection{Mujer/hombre de 60-65 años}

En el caso de las mujeres con edades entre 60 y 65 años, la diferencia entre alcanzar un nivel de educación de post-terciaria frente a algún otro nivel educativo puede explicar significativamente las diferencias en la probabilidad de participación, aunque la brecha se haya cerrado en favor de la educación terciaria completa después de 2010 (panel a). Este incremento en la probabilidad de participar de aquellos con terciaria completa se observa también para los hombres (panel b), señalando que en este periodo la gente tiene mayores niveles de educación que cohortes anteriores, lo que en parte puede explicar el incremento en la participación de los individuos con 60 años o más. 
a) Mujeres: Efecto estudia

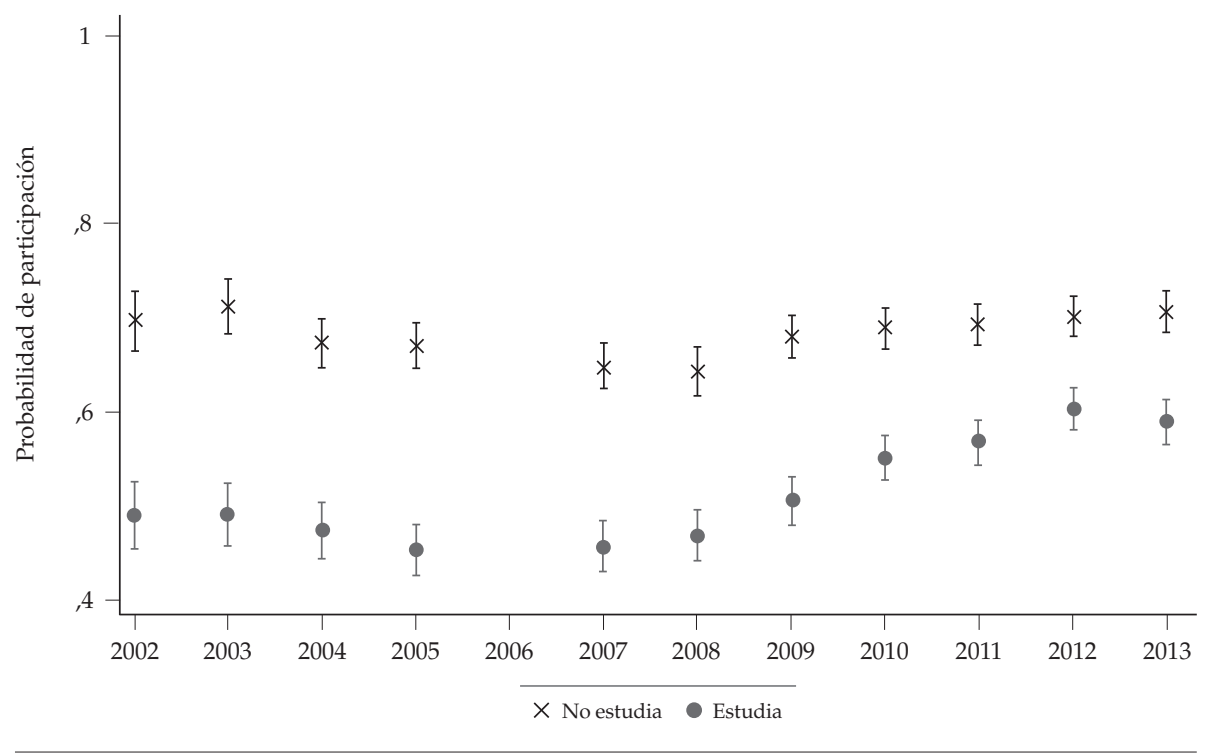

b) Hombres: Efecto estudia

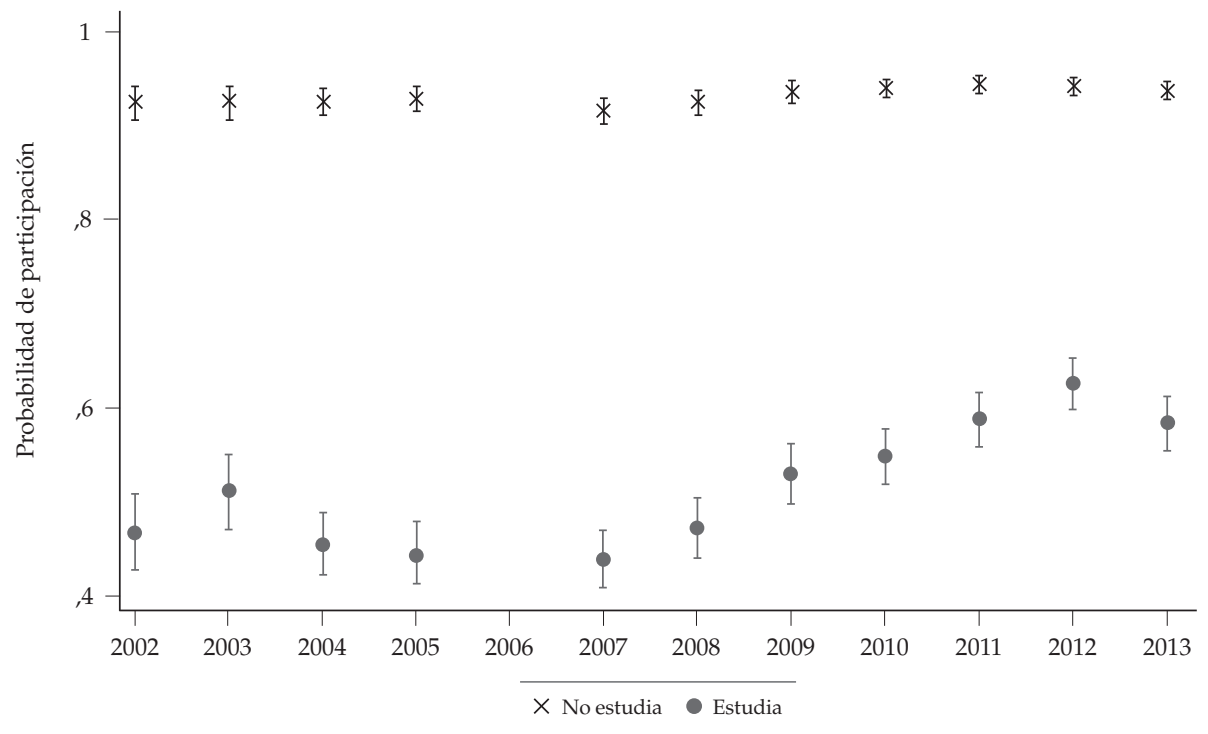


c) Mujeres: Efecto menores de 6 años

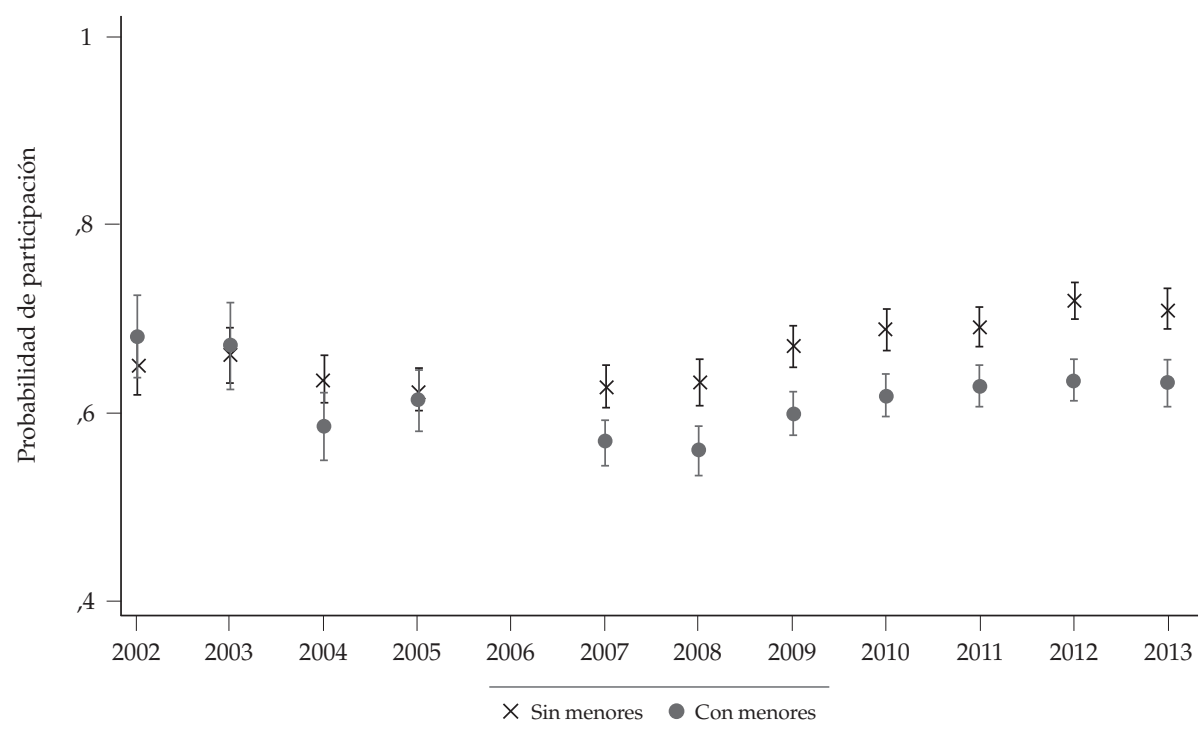

d) Hombres: Efecto menores de 6 años

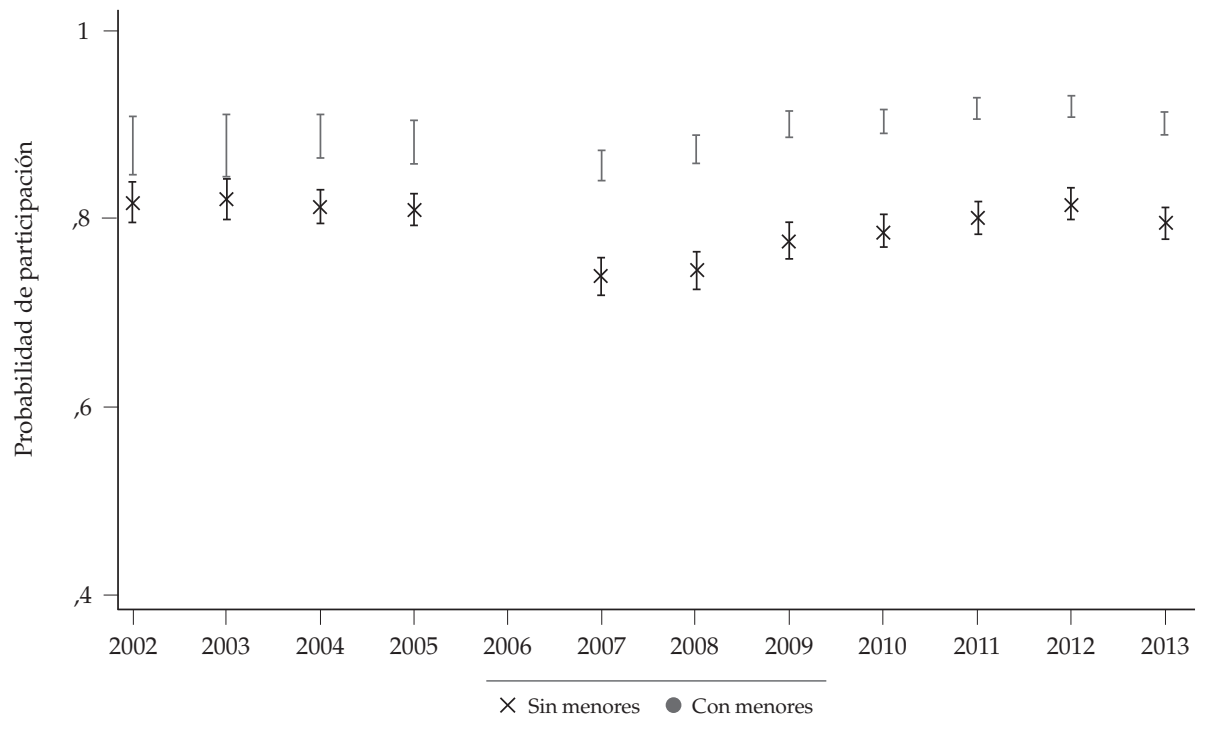

Figura 5, continúa página siguiente 
e) Mujeres: Efecto nivel educativo

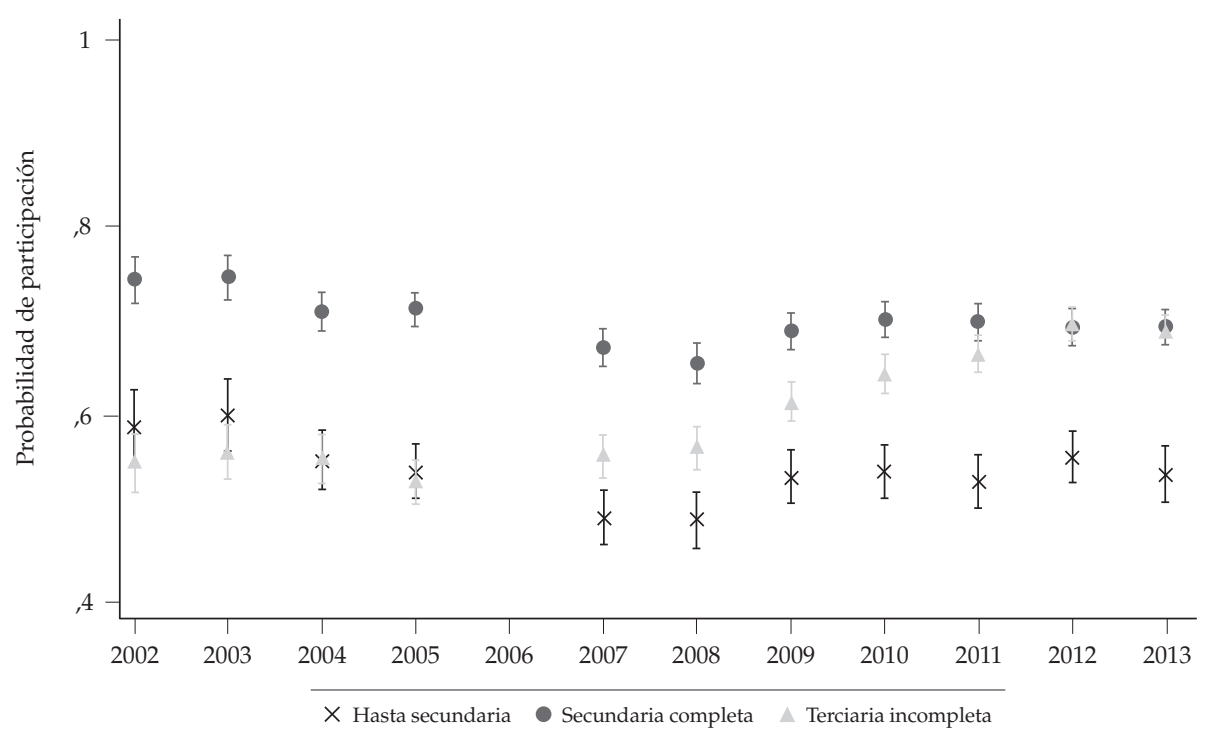

f) Hombres: Efecto nivel educativo

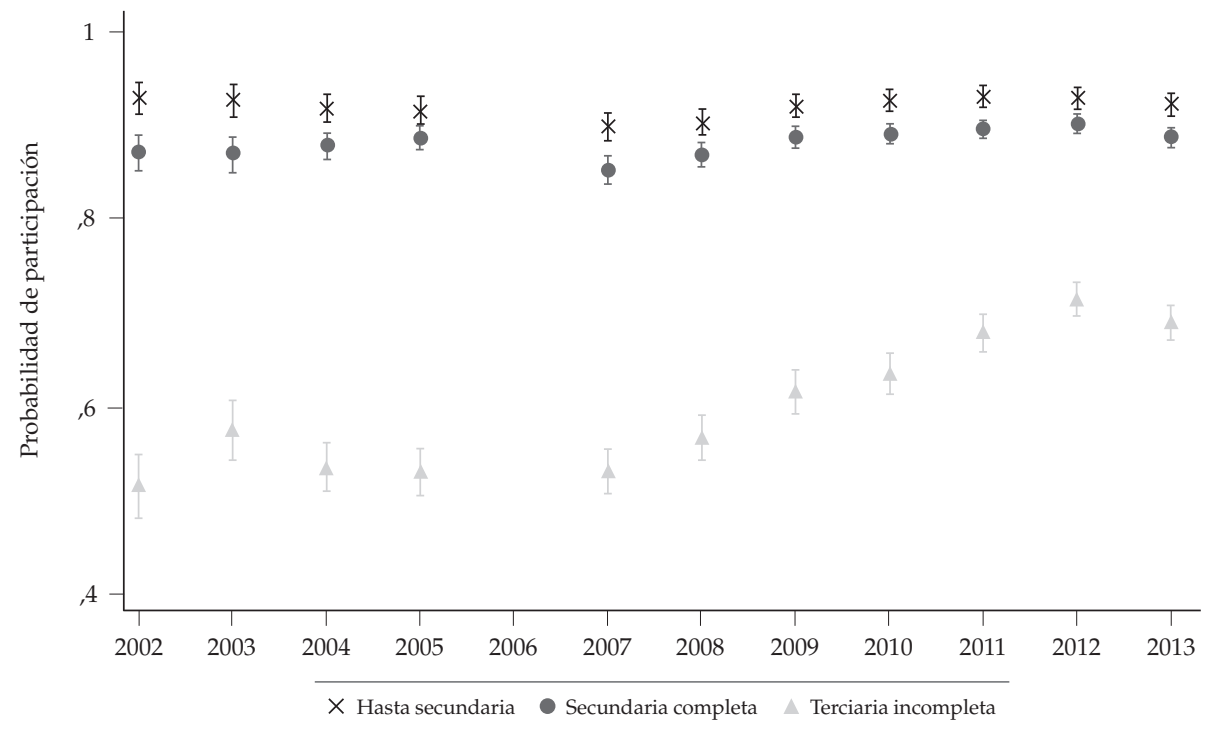


g) Mujeres: Efecto número de desempleados en el hogar

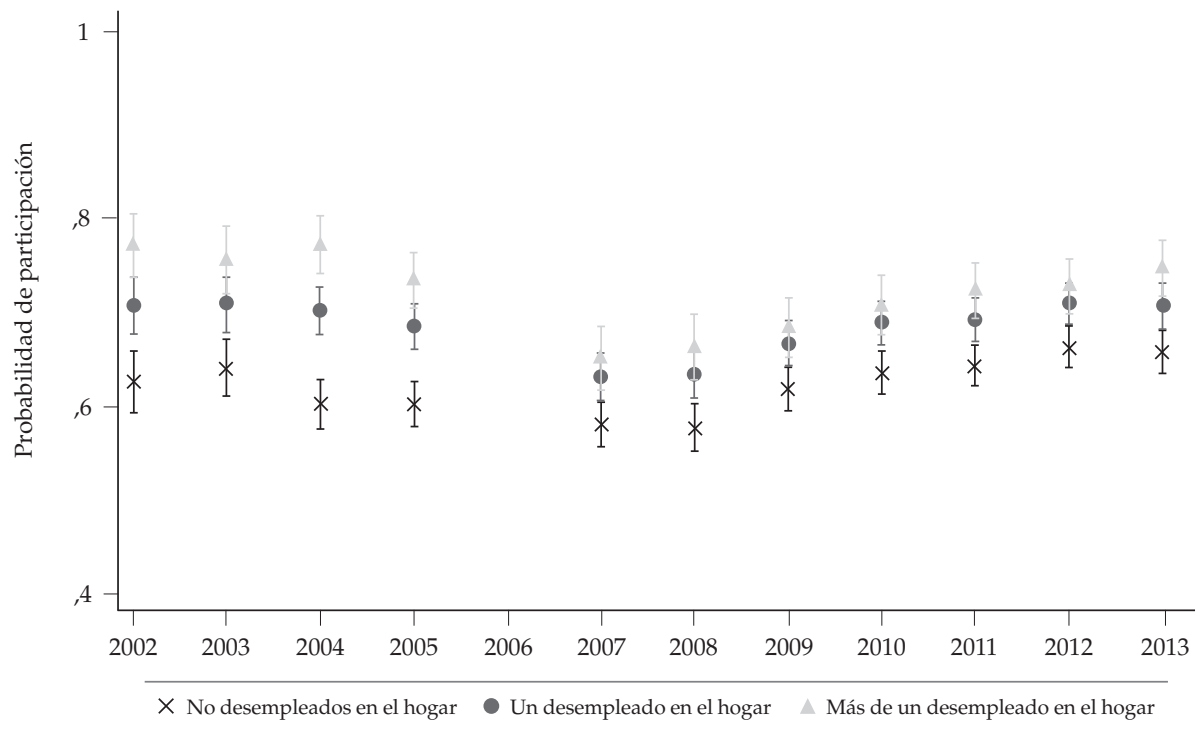

h) Hombres: Efecto número de desempleados en el hogar

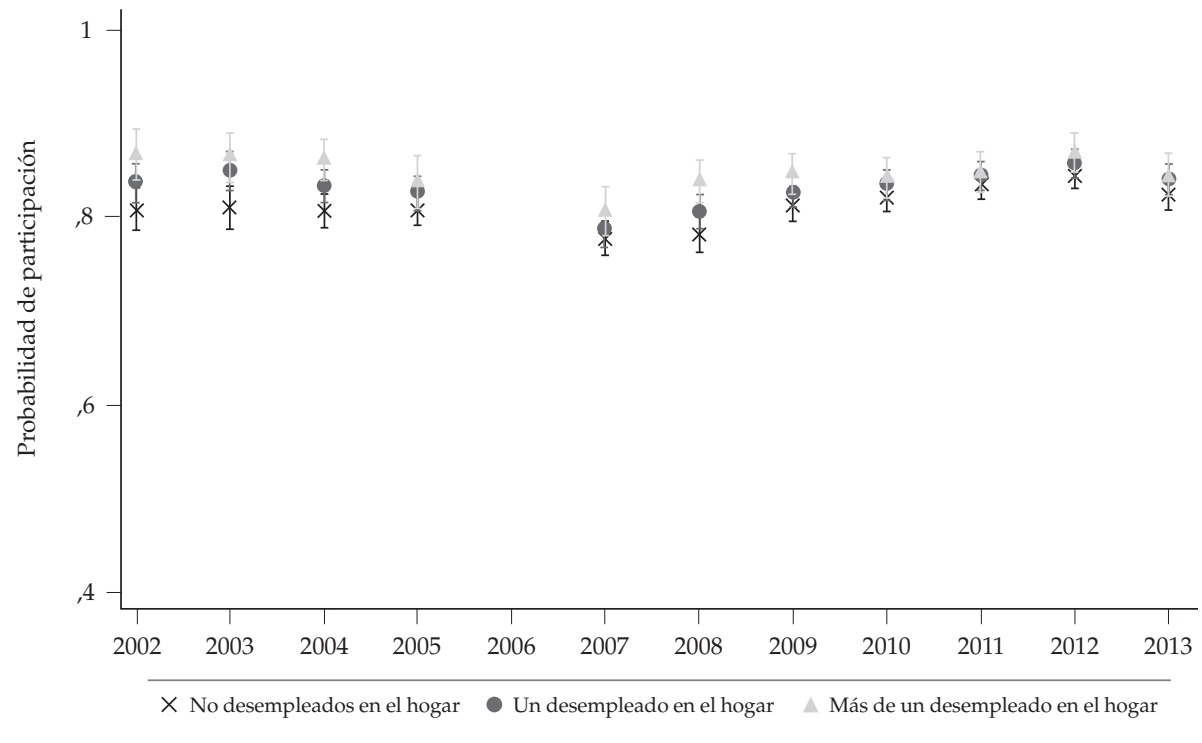

Figura 5: Probabilidad de participación promedio de mujeres y hombres de 20 a 23 años Fuente: Estimaciones de los autores.

Los paneles e y f de la figura 6 señalan que, al comparar la evolución en el periodo de estudio de las probabilidades de participación de mujeres y hombres con edades entre 60 y 65 años de edad, el hecho de no contar con ingreso 
por pensión en edad de retiro determina cerca de 30 puntos porcentuales más de probabilidad de participar para los hombres, y entre 5 y 10 puntos para las mujeres, aunque las diferencias no han cambiado significativamente a lo largo del periodo de estudio.

a) Mujeres: Efecto nivel educativo

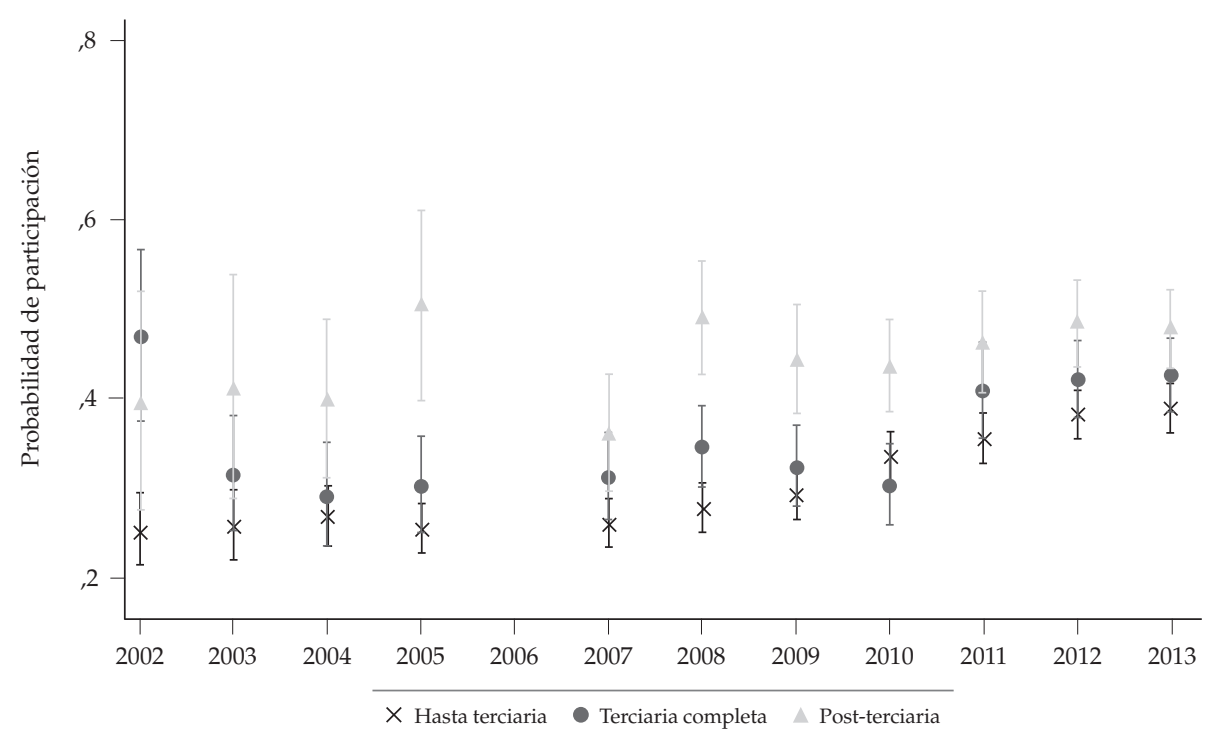

b) Hombres: Efecto nivel educativo

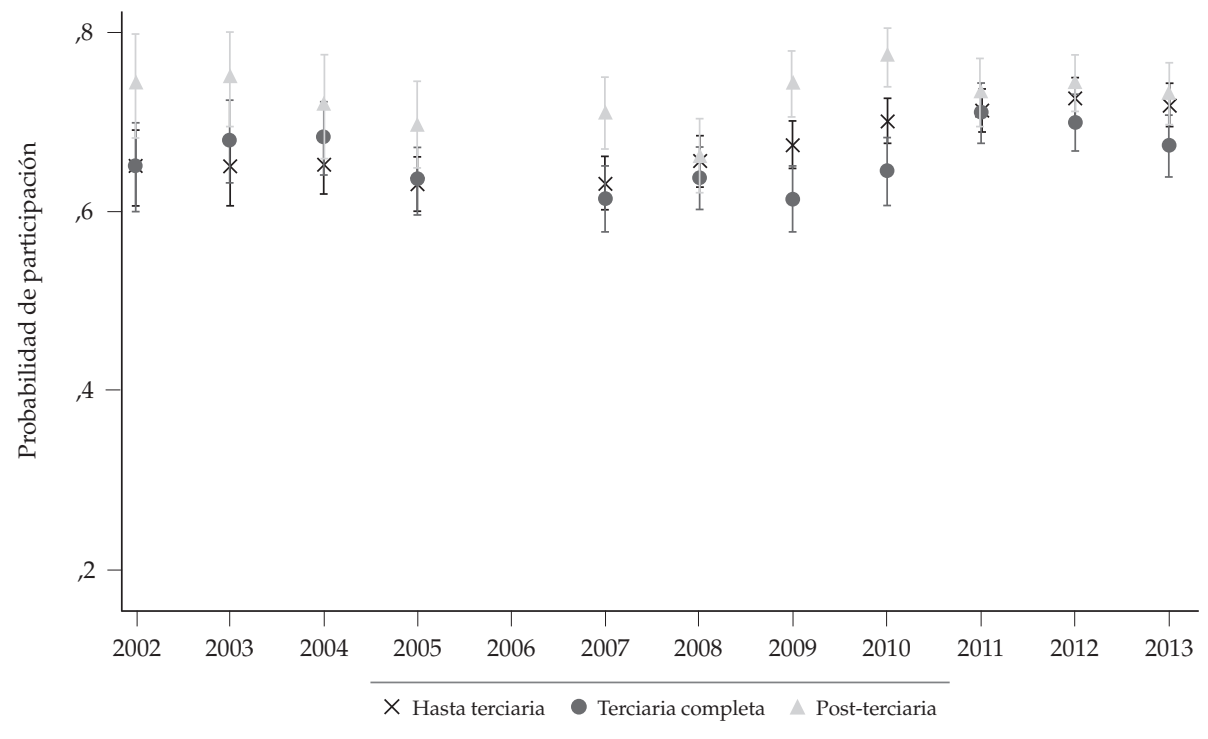


c) Mujeres: Efecto menores de 16 años

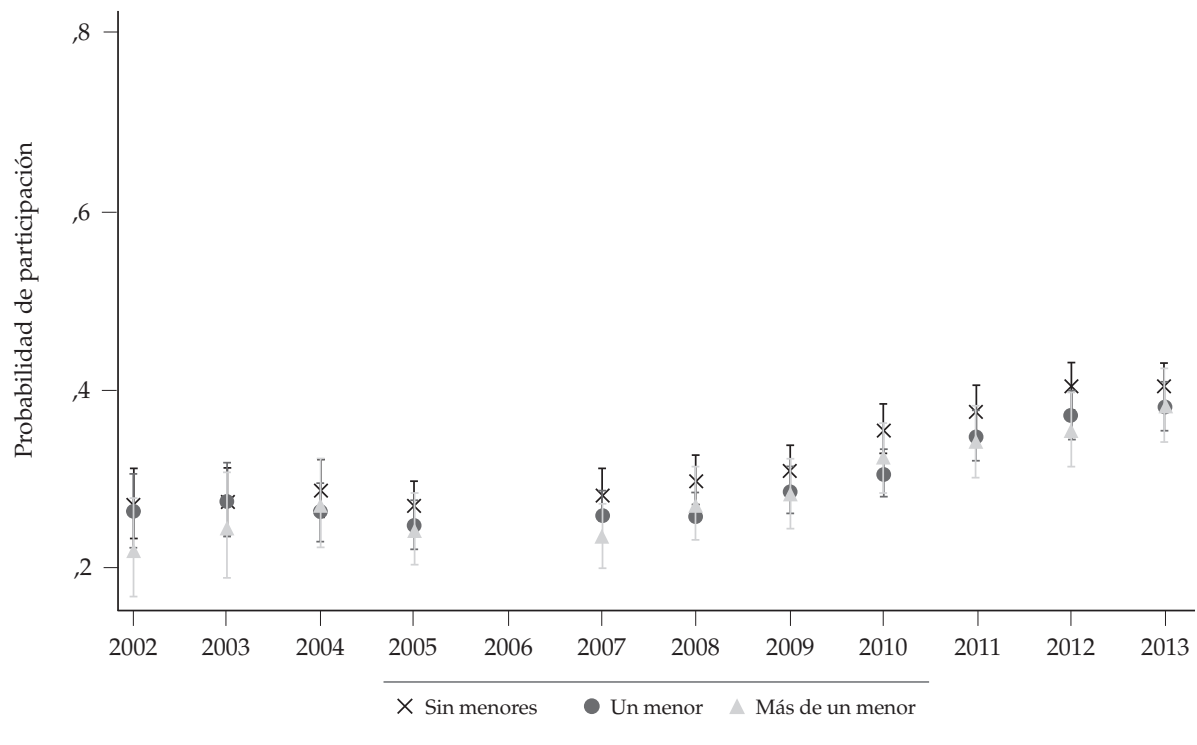

d) Hombres: Efecto menores de 16 años

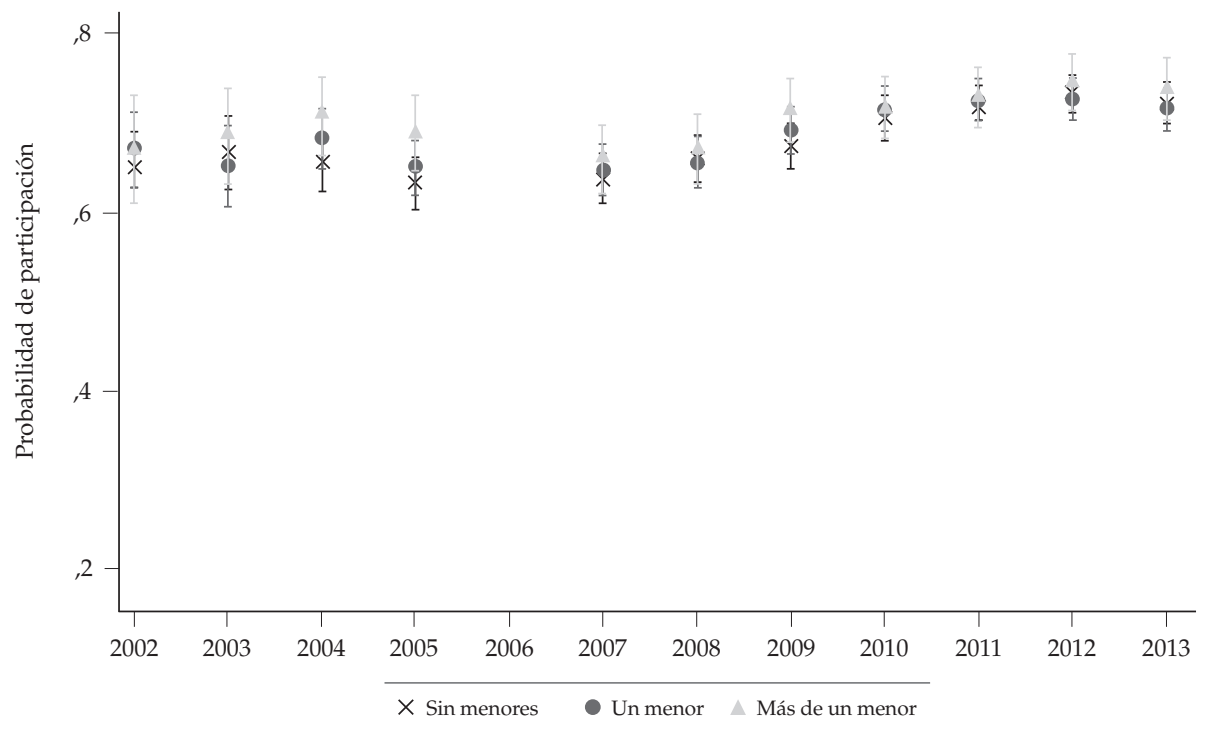

Figura 6, continúa página siguiente 
e) Mujeres: Efecto pensión

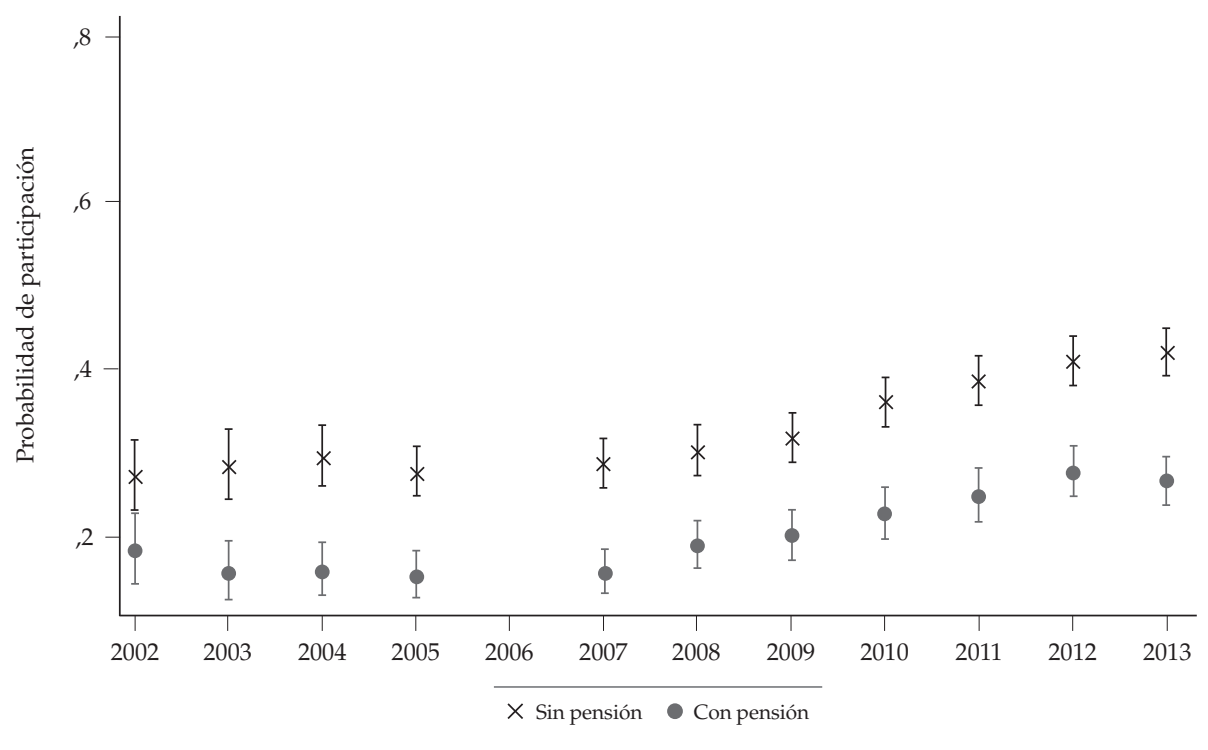

f) Hombres: Efecto pensión

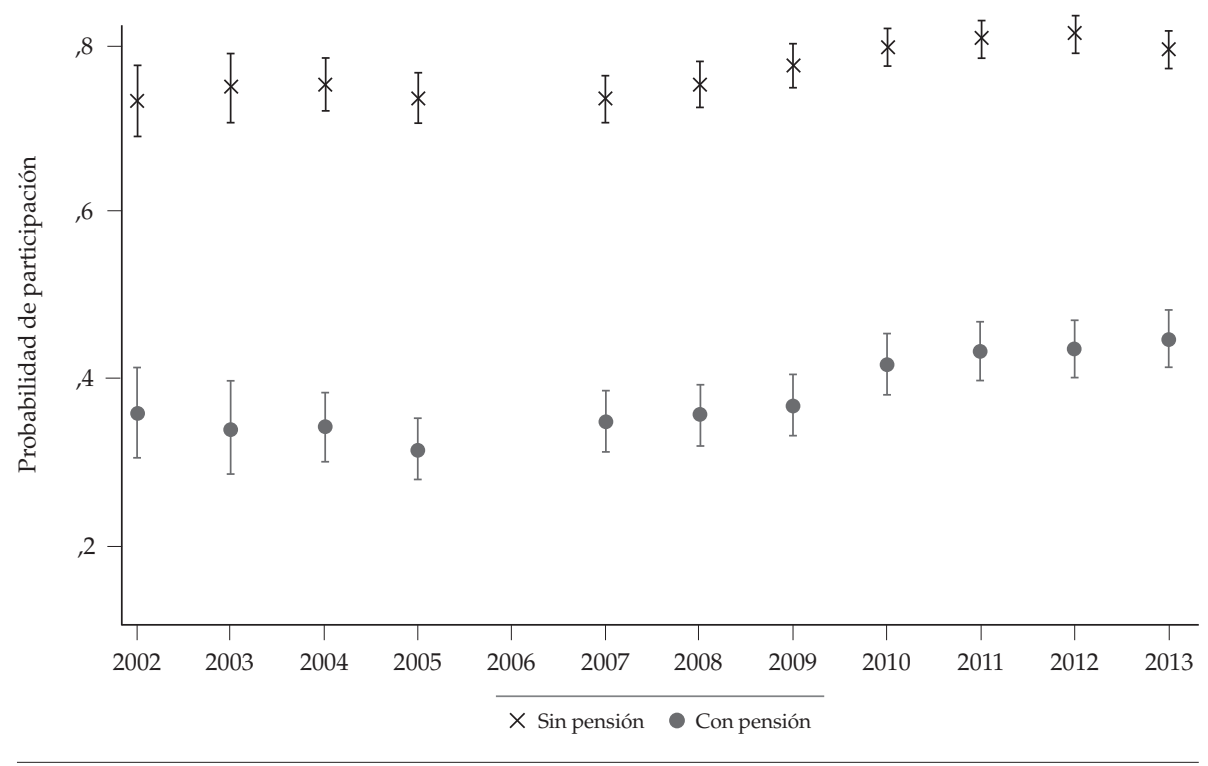


g) Mujeres: Efecto número de desempleados en el hogar

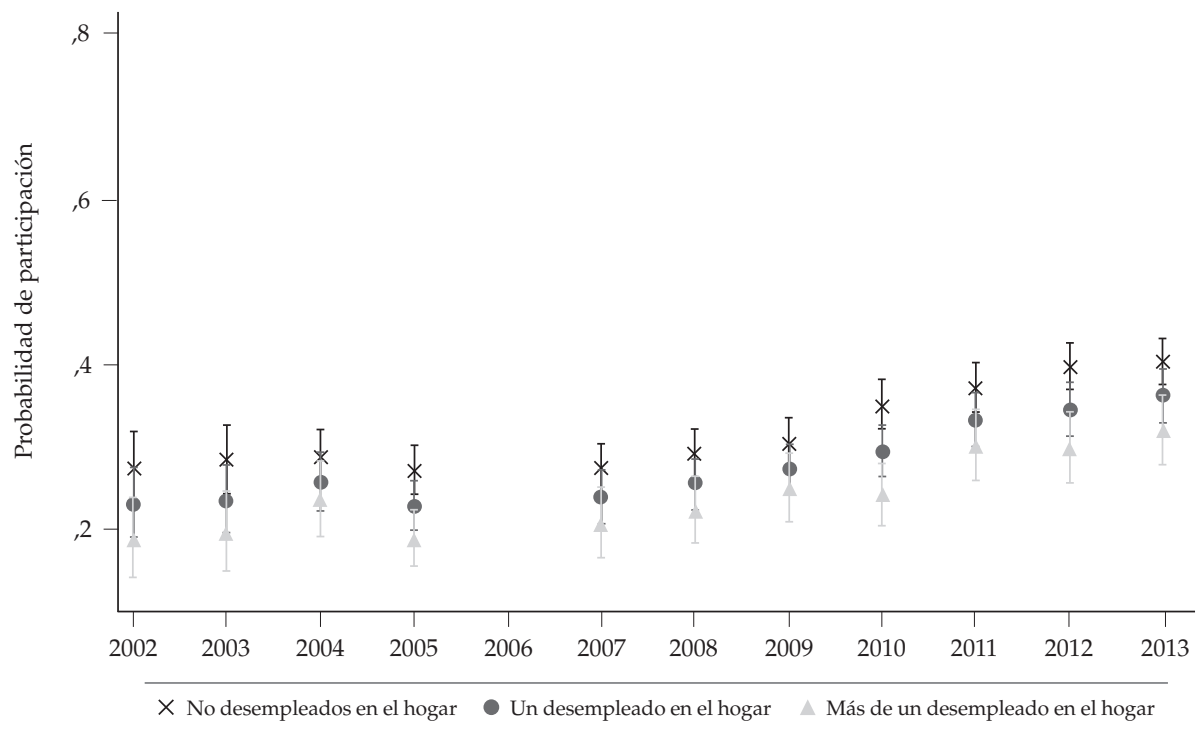

h) Hombres: Efecto número de desempleados en el hogar

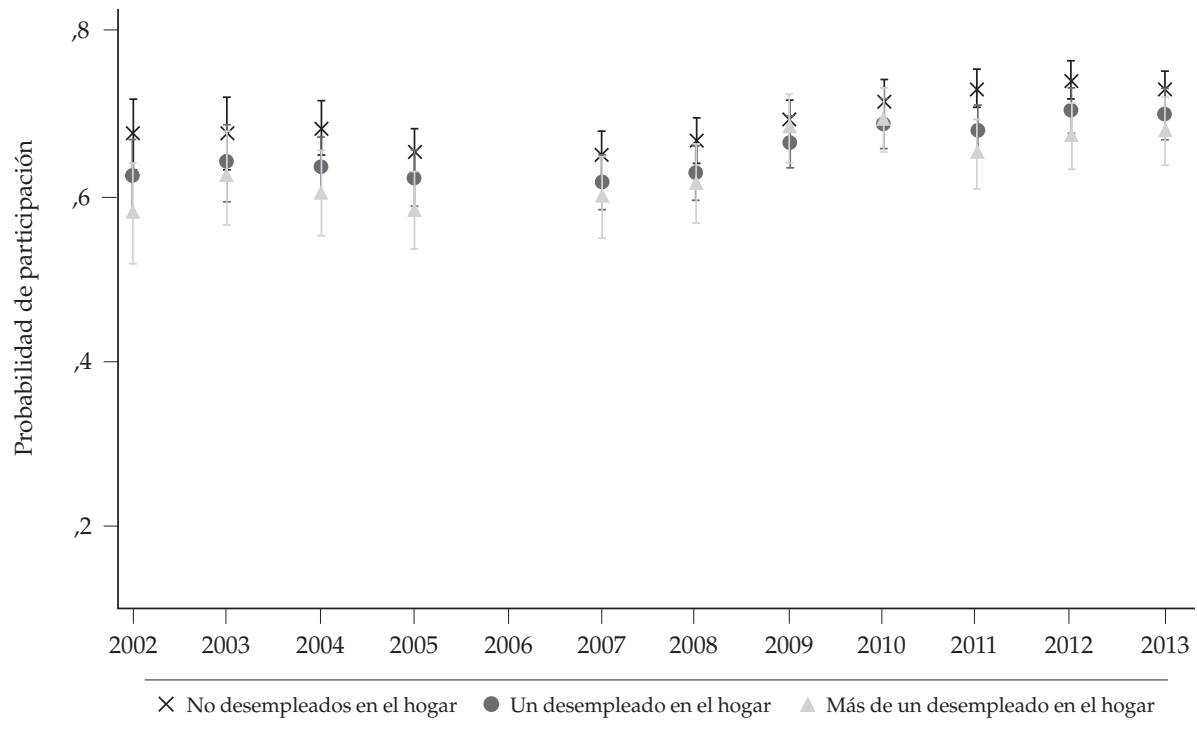

Figura 6. Probabilidad de participación de mujeres y hombres de 60 a 65 años

Fuente: Estimaciones de los autores. 
A pesar de tener efectos marginales significativos, el número de menores o de desempleados en el hogar no logran explicar diferencias significativas en la probabilidad de participar de los mayores de 59 años (paneles c, d y g, h).

\section{Conclusiones}

La evidencia señala que, en general, las mujeres, así como los hombres menores de 25 años y mayores de 59, son los que han determinado la dinámica de la tasa de participación laboral en los últimos 11 años. A partir de la unión de dos muestras de individuos de la ECH y la GEIH, se llevó a cabo el ejercicio de hallazgo de los determinantes de la participación laboral en Colombia para cada uno de estos grupos en el periodo 2002-2013, utilizando un modelo empírico tipo probit que representa el problema estático de extensión de la oferta laboral. Como complemento de este primer análisis, y con el propósito de establecer el aporte real de estos determinantes a la dinámica de las tasas de participación en el periodo de estudio, se crearon perfiles de probabilidad de participación para subgrupos de individuos en cada uno de los grupos etarios elegidos para este estudio. Se calcularon probabilidades de participación condicionadas a los diferentes determinantes en cada uno de los años del periodo 2002-2013 y se realizaron comparaciones utilizando intervalos de confianza del $90 \%$.

Para el total nacional, el nivel educativo alcanzado por el individuo, la presencia de dependientes en el hogar (sean menores de edad o adultos mayores), el número de desempleados que allí habitan, la situación escolar actual y el ingreso del resto de los miembros de la casa son determinantes importantes de la probabilidad de participar. Alcanzar niveles educativos superiores a la secundaria completa es especialmente relevante para las mujeres casadas en el rango de edad de 25 a 35 años, las mujeres jóvenes menores de 25 años, y las mujeres con más de 59 años.

De otro lado, el efecto de la presencia de menores funciona en sentido inverso entre mujeres casadas y no casadas; mientras que para las primeras este factor tiene un efecto negativo sobre la probabilidad de participación, para las segundas es positivo. En el primer caso, la posibilidad de compartir responsabilidades hace que la presencia de menores de 16 años incremente el costo marginal de ofrecer tiempo en el mercado de trabajo. En el segundo caso, la presencia de menores crea una dependencia económica y, por el contrario, reduce dicho costo.

En el caso de los individuos jóvenes menores de 19 años, la presencia de niños de hasta 6 años crea dependencia económica, en especial para los hombres; mientras que para aquellos con edades entre 19 y 24 años, dicha 
presencia crea dependencia no económica en las mujeres (desincentivándolas a participar) y dependencia económica en los hombres. Para ambos grupos la situación escolar actual (si no estudia o estudia), determina en promedio si el individuo participa o no participa. Al contrario de lo esperado, el haber alcanzado niveles de educación inferiores a la secundaria completa tiene un efecto positivo sobre la probabilidad de participación de los hombres jóvenes.

En el grupo de mayores de 59 años, se detectaron efectos cohorte asociados con la educación y el cubrimiento del sistema de seguridad social en pensiones: una cohorte con niveles de educación más altos tiene mayor probabilidad de participación; una cohorte con mayor número de individuos que superan los 59 años tiene mayor probabilidad de participar si no está cubierta por el sistema de seguridad social. En este último caso, la alta evasión del sistema formal de ahorro para la vejez, junto con una baja propensión marginal a ahorrar en otros mecanismos de ahorro informal hacen más costoso no ofrecer tiempo en el mercado de trabajo y aplazan el retiro.

El efecto del número de desempleados en el hogar refleja el mecanismo worker-added effect o trabajador adicional para las mujeres con edades entre 19 y 59 años. Para mujeres y hombres jóvenes menores de 19 años e individuos mayores de 59, el mecanismo parece ser el discouraged-worker effect o trabajador desalentado, cuya explicación puede fundamentarse en la hipótesis de una reducción en la sustitución laboral intra-familiar en el periodo pos crisis. Por otra parte, el efecto marginal del logaritmo que tiene que ver con el ingreso del resto del hogar refleja que ante diferencias del $10 \%$ en este, la probabilidad de participar se reduce entre tres y siete puntos porcentuales.

La comparación en cada perfil determinó que el incremento después de 2007 en la tasa de participación de las mujeres de 25 a 59 años estuvo fuertemente asociado con las mayores probabilidades de participación de las mujeres con menor nivel de educación, ante una menor presencia de menores en el hogar. Por su parte, la dinámica de esta tasa para las mujeres menores de 25 años se relaciona con una reducción en el número de desempleados en el periodo 2002-2005 (mejoramiento de la situación del mercado de trabajo) y un incremento en el nivel educativo alcanzado después de 2008. Este último factor es importante también para los hombres jóvenes en este rango de edad.

La comparación de perfiles para mujeres y hombres menores de 25 años muestra que la dinámica creciente de la participación puede asociarse también con una reducción en la sustitución entre el uso del tiempo en el estudio y en el mercado de trabajo, en especial cuando se alcanzan niveles de educación terciaria incompleta. Sin embargo, en el caso de los menores de 19 años, a pesar de alcanzar niveles de educación terciaria, el costo del uso del tiempo en 
actividades no laborales es lo suficientemente alto para tomar la decisión de participar y abandonar sus estudios.

Por último, y a pesar de que la posibilidad de contar con un ingreso por pensión explica las mayores diferencias en la participación de los individuos mayores de 59 años, la comparación de los perfiles condicionados muestra que el haber alcanzado un nivel de educación igual o superior a la terciaria completa ha incrementado de forma significativa la probabilidad de participación, inclusive la de aquellos con pensión.

Estos resultados traen consigo varios retos de política pública, si se espera que los mayores niveles de participación se reflejen en mayores niveles de empleo, crecimiento y productividad. En primer lugar, el sistema de educación superior debe buscar mecanismos que reduzcan el costo marginal de usar el tiempo en actividades educativas y, al menos, le permitan al individuo considerar la opción de estudiar y trabajar al mismo tiempo. El riesgo de abandono parece encontrarse en los primeros años, de acuerdo con los resultados. Más allá de estos mecanismos, solo podrán lograrse altos niveles de productividad si, a pesar de estudiar y trabajar al mismo tiempo ante la reducción en la sustitución del uso del tiempo, la educación recibida por estos individuos asegure ciertos niveles de calidad que les permitan participar de manera exitosa en el mercado de trabajo.

En segundo lugar, la alta informalidad laboral y empresarial, así como la aguda evasión del sistema de seguridad social formal, retrasan el retiro voluntario de trabajadores con bajos niveles de productividad, asociados con no haber alcanzado niveles de educación superior. Es positivo que haya mayores niveles de participación de individuos en edad de retiro con niveles de educación superior, pues es posible que su senda de productividad aún se encuentre en la parte creciente, pero no lo es si la decisión de retiro se aplaza únicamente porque no se cuenta con un mecanismo de cobertura para la vejez.

Por último, y tal como se ha concluido en los trabajos similares y anteriores a este, los mayores niveles de participación femenina, asociados con una menor dependencia no económica, y los de educación alcanzados, exigen políticas que disminuyan la brecha de ingreso salarial explicada por factores diferentes a la productividad.

\section{Referencias}

Amador, D., Peña, X., \& Bernal, R. (2013). The rise in female participation in Colombia: Fertility, marital status or education? Ensayos sobre Politica Económica, 31, 54-63. 
Arango, L. E., \& Posada, C. E. (2002). La participación laboral en Colombia. Borradores de Economía 217. Bogotá: Banco de la República.

Arango, L. E., Posada, C. E., \& Charry, A. (2003). La participación laboral en Colombia según la nueva encuesta: ¿cambian sus determinantes? Borradores de Economía 250. Bogotá: Banco de la República.

Arriagada, I. (1997). Realidades y mitos del trabajo femenino urbano en América Latina. Serie Mujer y Desarrollo 21. España: Cepal.

Chiappori, P. A. (1992). Collective Labor Supply and Welfare. Journal of Political Economy, 100(3), 437-467.

Guataquí, J. C., Rodríguez-Acosta, M., \& García-Suaza, A. F. (2009). Ahorro para el Retiro en Colombia: Patrones y Determinantes. Serie Documentos de Trabajo 72. Bogotá: Universidad del Rosario.

Heckman, J. J. (1974). Shadow prices, market wages and labor supply. Econométrica, 42(4), 679-694.

Heckman, J. J. (1978). Dummy Endogenous Variables in a Simultaneous Equation System. Econométrica, 46(4), 931-959.

Heckman, J. J. (1993). What has Been Learned About Labor Supply in the Past Twenty Years? The American Economic Review, 83(2), 116-121.

Heckman, J. J., \& Willis, R. J. (1977). A Beta-logistic Model for the Analysis of Sequential Labor Force Participation by Married Women. Journal of Political Economy, 85(1), 27-58.

Hotchkiss, J. L., \& Robertson, J. C. (2006). Asymmetric Labor Force Participation Decisions over the Business Cycle: Evidence from U.S. Microdata. Working Paper (2006-8). Atlanta: Federal Reserve Bank of Atlanta.

Juhn, C., \& Potter, S. (2006). Changes in Labor Force Participation in the United States. Journal of Economic Perspectives, 20(3), 27-46.

López, H. (1985). El comportamiento de la oferta laboral y la tasa de desempleo: indeterminación teórica e incertidumbre empírica. Lecturas de Economía 16, 41-62.

Mincer, J. (1974). Schooling, Experience and Earnings. Nueva York: National Bureau of Economic Research.

Mulligan, C. B. (1998). Substitution over Time: Another Look at Life-Cycle Labor Supply. NBER Macroeconomics Annual, 13, 75-134.

Núñez, J., \& González, N. (2011). Colombia. En M. Sánchez, \& P. Sauma, Vulnerabilidad económica externa, protección social y pobreza en América Latina (pp. 209-262). Santiago-Quito-Nueva York: CEPAL-FLACSO-UN/DESA.

Ribero, R., \& Meza, C. (1997). Determinantes de la participación laboral de hombres y mujeres en Colombia: 1976-1995. Archivos de Economía 63. Bogotá: Departamento Nacional de Planeación. 
Sánchez, F., \& Núñez, J. (2003). A dynamic analysis of human capital, female work-participation, returns to education and changes in household structure in urban Colombia, 1976-1998. Colombian Economic Journal, 1(1), 110-149. Santamaría, M., \& Rojas, N. (2001). La participación laboral: ¿qué ha pasado y qué podemos esperar? Archivos de Economía 146. Bogotá: Departamento Nacional de Planeación.

Tenjo, J., \& Ribero, R. (1998). Participación, desempleo y mercados laborales en Colombia. Archivos de Economía 81. Bogotá: Departamento Nacional de Planeación. 


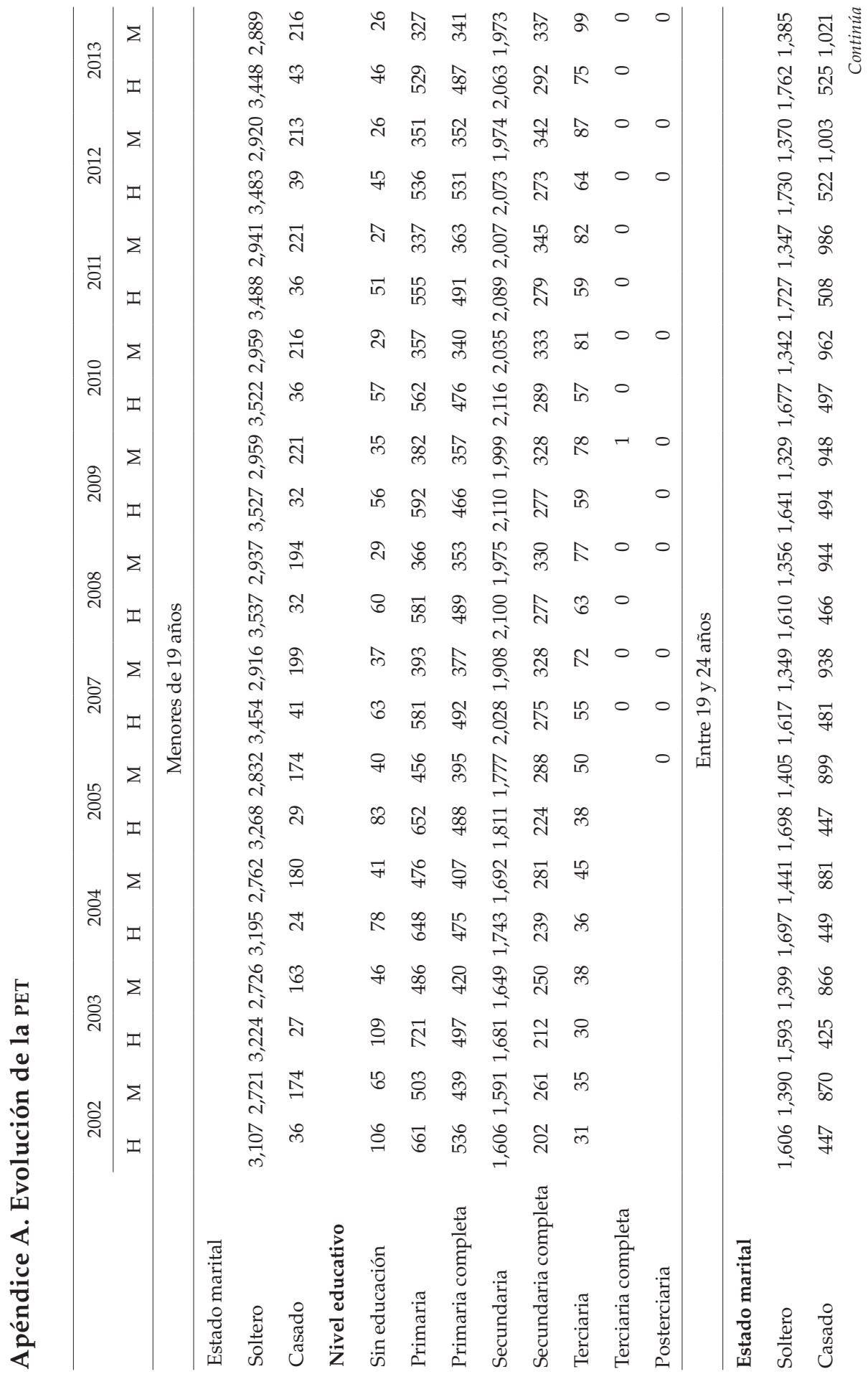




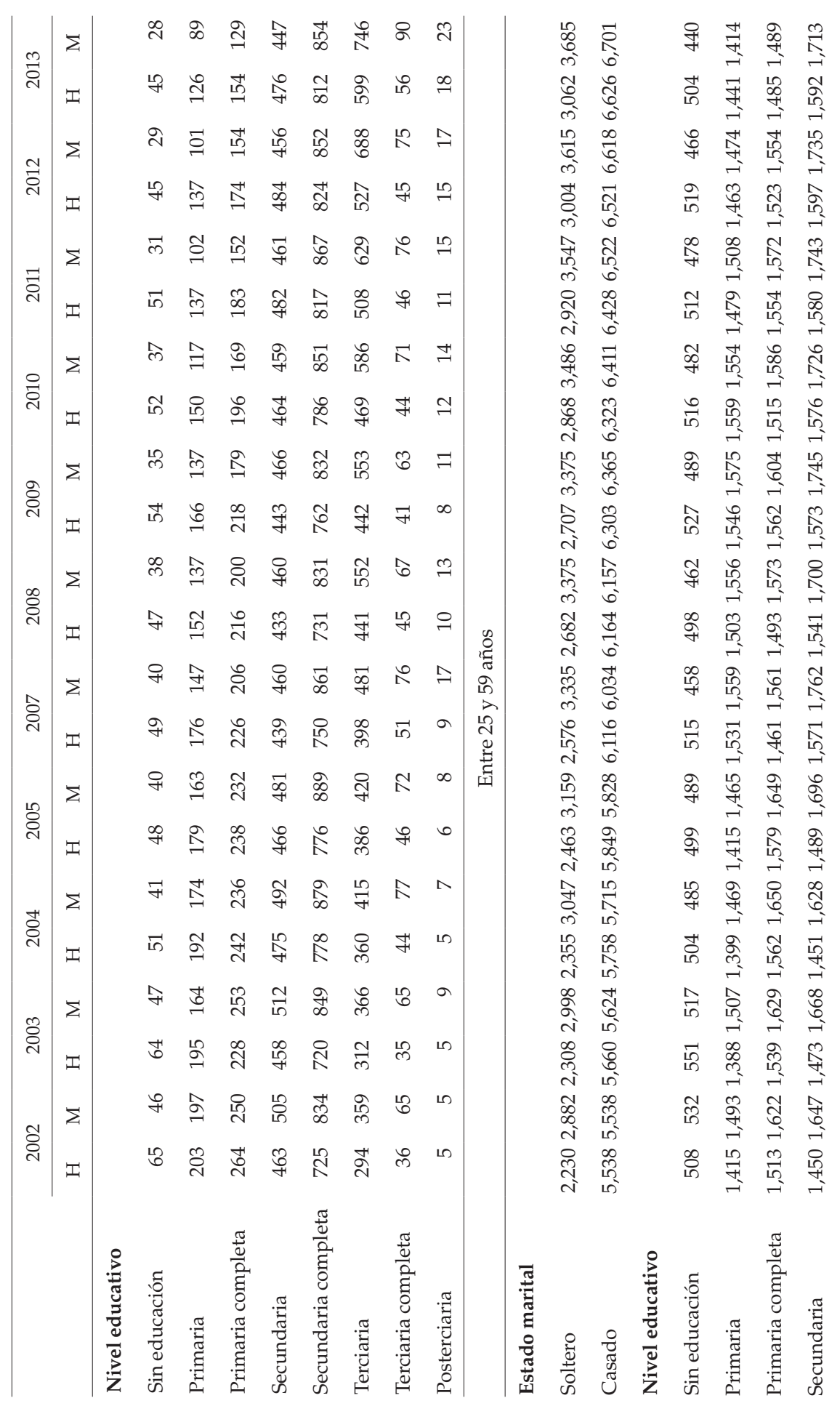




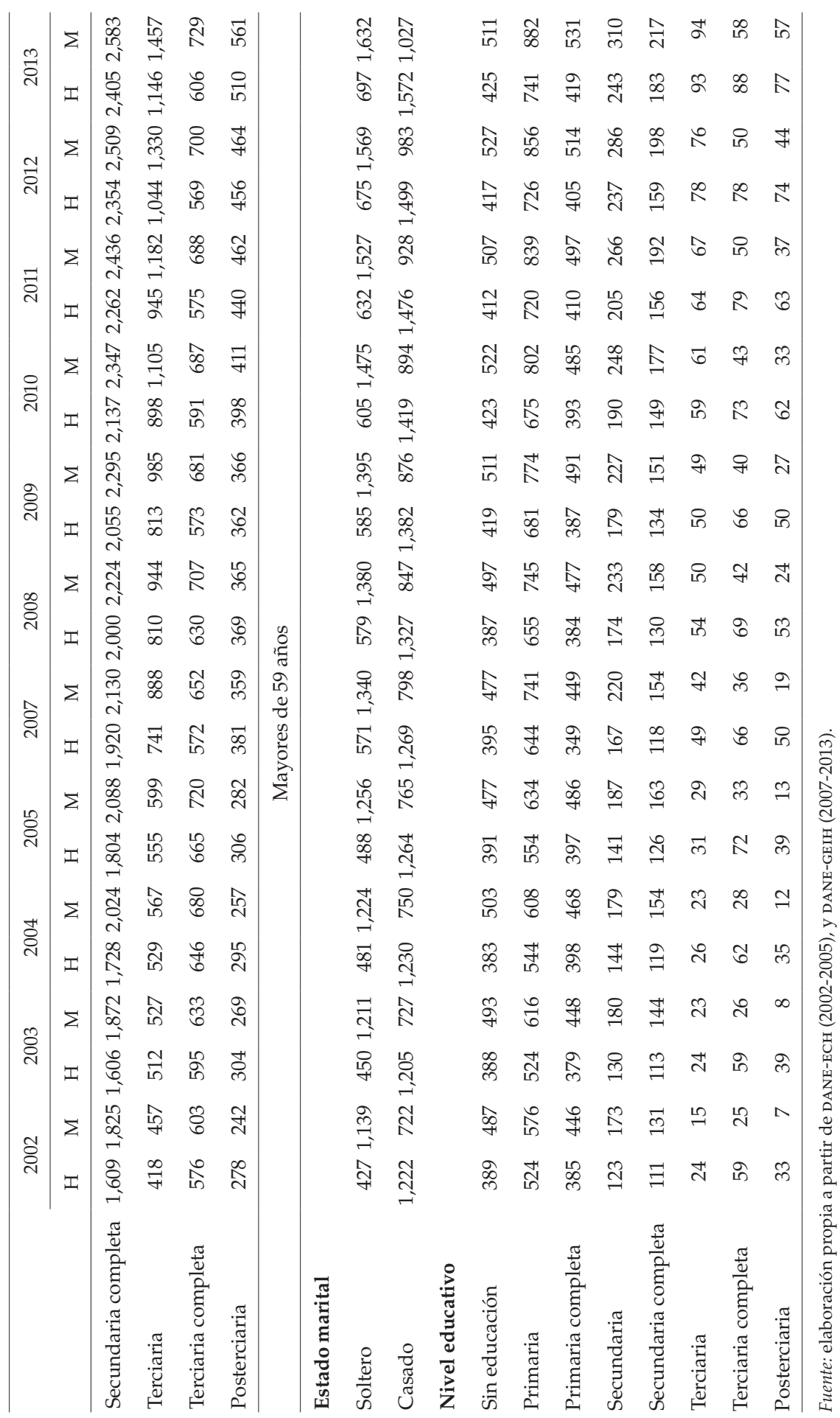




\section{Apéndice B. Problemas de continuidad histórica de la encuesta de hogares en Colombia}

El Departamento Administrativo Nacional de Estadística (DANE) ha venido desarrollando encuestas de hogares desde los años setenta, con el fin de proporcionar estadísticas relacionadas con la situación sociodemográfica y económica de la población colombiana. En 1976, se dio inicio a la Encuesta Nacional de Hogares (ENH), que se realizó, en forma trimestral, para cuatro ciudades (Bogotá, Cali, Medellín y Barranquilla) y, en forma semestral, para tres más (Bucaramanga, Manizales y Pasto). De manera periódica, se fueron incluyendo áreas rurales, municipios circundantes y otras ciudades (Pereira, Cúcuta, Ibagué, Montería, Cartagena y Villavicencio), llegando a tener, en el año 2000, una cobertura nacional con cuatro desagregaciones geográficas (zona urbana, rural, grandes regiones y departamental).

En 2001, el DANE sustituyó la ENH, por la Encuesta Continua de Hogares $(\mathrm{ECH})$, la cual se aplicaba de forma mensual y continua en cada semana del mes. Su cubrimiento aproximado en el mes era de 44400 hogares y abarcaba las 13 áreas metropolitanas y más de 240 municipios en todo el país, proporcionando información representativa en el ámbito nacional, cabecera-resto, departamental (medición anual) y para algunas capitales de departamento. A partir del tercer trimestre de 2006, la ЕсH fue reemplazada por la Gran Encuesta Integrada de Hogares (GEIH), la cual amplió la cobertura a las 11 ciudades capitales restantes, lo que significó un incremento de 17600 hogares. Adicional al cambio del marco muestral, instituyó el uso de dispositivos móviles como sistema de recolección de la información y cambió de informante idóneo a informante directo ${ }^{11}$. A pesar del cambio en el marco muestral entre la ECH y la GEIH, los elementos esenciales del diseño muestral se mantienen, es decir, son muestras probabilísticas, estratificadas, de conglomerados, multietápicas y autoponderadas (para las 13 ciudades con sus áreas metropolitanas y actualmente con las capitales de departamento), lo que indica que no se puede seguir a un mismo individuo a lo largo de los diferentes periodos, dado que su selección para hacer parte de la muestra es aleatoria en cada periodo.

11 Entendiendo informante idóneo como la persona mayor de 18 años de edad que se siente en capacidad de dar información de un miembro del hogar diferente a él y que sea distinto de empleado del servicio doméstico o pensionista, generalmente los jefes de hogar. El informante directo, hace referencia a que cada miembro del hogar mayor de 10 años, en zona rural, y de 12, en zona urbana, debe responder de manera individual las preguntas de la encuesta. 
Los cambios entre las encuestas generaron inconvenientes en la compatibilidad temporal de los indicadores de mercado laboral producidos por las dos encuestas; por lo que el DANE, en 2008, contrata a la Comisión de Expertos Independientes (CEI) para llevar a cabo la Misión para el Empalme de Series de Empleo, Pobreza y Desigualdad (MESEP). La comisión concluyó que los cambios que surgieron de la ECH a la GEIH afectan los instrumentos de medición, el diseño y composición de la muestra sobre la cual se recolecta la información, por lo que realizó un empalme de las series de TGP, TO y TD mediante el uso de factores de ajuste, calculados empleando información de la ECH, la GEIH y una encuesta paralela que usaba los instrumentos de la ECH y que fue realizada en 2008. 
